Supporting Information

\title{
Elucidating Secondary Metal Cation Effects on Nickel Olefin Polymerization Catalysts
}

\author{
Thi V. Tran, Lucas J. Karas, Judy I. Wu, Loi H. Do* \\ Department of Chemistry, University of Houston, 4800 Calhoun Rd., Houston, Texas, 77204, \\ United States
}

Email:loido@uh.edu

TABLE OF CONTENTS

$\underline{\operatorname{Page}(\mathbf{s})}$

Experimental

Scheme S1

Synthesis of Ni1-Ni3

S3

Synthesis and Characterization

S4-S5

Metal Binding Studies

UV-vis Absorption Spectroscopy: Titrations $\quad$ S6

Figure S1

UV-vis Titration Plots of Ni3 $+\mathrm{MBAr}_{4}$

S7

UV-vis Absorption Spectroscopy: Job Plots $\quad$ S8

Table S1

Data and Calculations for Job Plot of Ni3 $+\mathrm{LiBAr}_{4}$

S9

Figure S2

Job Plot of Ni3 + $\operatorname{LiBAr}_{4}$

S9

Table S2

Data and Calculations for Job Plot of Ni3 $+\mathrm{NaBAr}_{4}$

$\mathrm{S} 10$

Figure S3

Job Plot of Ni3 + $\mathrm{NaBAr}_{4}$

$\mathrm{S} 10$

Table S3

Data and Calculations for Job Plot of Ni3 $+\mathrm{KBAr}_{4}$

$\mathrm{S} 11$

Figure S4

Job Plot of Ni3 $+\mathrm{KBAr}_{4}$

$\mathrm{S} 11$

Table S4

Data and Calculations for Job Plot of Ni3 $+\mathrm{CsBAr}_{4}$

$\mathrm{S} 12$

Figure S5

Job Plot of $\mathbf{N i 3}+\mathrm{CsBAr}_{4}{ }_{4}$

$\mathrm{S} 12$

\section{Polymerization Studies}

Figure S6

Table S5

Figure S7

Effect of Temperature on Activity, Branching, and MW

$\mathrm{S} 13$

Polymerization by Ni3/MBAr ${ }_{4}$ in Different Solvents $\quad$ S14

Table S6

Effect of Solvent on Ni3-M

$\mathrm{S} 15$

Polymerization by Ni3-Li at Various Temperatures $\quad$ S16

Figure S8

Effect of Temperature on Ni3-Li and its Optimization

S16

Table S7

Polymerization by Ni3-Li in Various Solvents

S17

Table S8

Polymerization by Ni3 + Various Equiv. of Salt

$\mathrm{S} 18$

Figure S9

Effect of Salt Equiv. on Polymerization by Ni3

$\mathrm{S} 18$

Table S9

Polymerization by Ni3 + Different Li Salts

S19

Table S10

Time Study of Polymerization by Ni3-Li

S19

Table S11

Comparison of Reported Ni Catalysts

S20

Table S12

Comparison of Reported Ni Catalysts at High Temperature

S21

Structural Studies

Figure S10

Topographic Steric Maps of Ni1' and Ni2

S22

Figure S11

Activation Studies

Topographic Steric Maps Ni3-M 
Figure S12 $\quad{ }^{31} \mathrm{P}$ NMR Spectra of $\mathbf{N i 3}+\mathrm{B}\left(\mathrm{C}_{6} \mathrm{~F}_{5}\right)_{3}, \mathrm{RT} \quad \mathrm{S} 24$

Figure S13 $\quad{ }^{31} \mathrm{P}$ NMR Spectra of Ni3/LiBAr ${ }^{\mathrm{F}_{4}}+\mathrm{B}\left(\mathrm{C}_{6} \mathrm{~F}_{5}\right)_{3}$, RT $\quad$ S25

Figure S14 $\quad{ }^{31} \mathrm{P}$ NMR Spectra of Ni3/B $\left(\mathrm{C}_{6} \mathrm{~F}_{5}\right)_{3}+\mathrm{LiBAr}^{\mathrm{F}}{ }_{4}, \mathrm{RT} \quad \mathrm{S} 25$

Figure S15 $\quad{ }^{31} \mathrm{P}$ NMR Spectra of Ni3/LiBAr ${ }^{\mathrm{F}}{ }_{4}+\mathrm{B}\left(\mathrm{C}_{6} \mathrm{~F}_{5}\right)_{3}, 50{ }^{\circ} \mathrm{C} \quad \mathrm{S} 26$

Figure S16 $\quad{ }^{31} \mathrm{P}$ NMR Spectra of $\mathrm{Ni3} / \mathrm{NaBAr}_{4}+\mathrm{B}\left(\mathrm{C}_{6} \mathrm{~F}_{5}\right)_{3}$, RT $\quad$ S26

Figure S17 $\quad{ }^{31} \mathrm{P}$ NMR Spectra of Ni3/KBAr ${ }_{4}+\mathrm{B}\left(\mathrm{C}_{6} \mathrm{~F}_{5}\right)_{3}$, RT $\quad$ S27

Figure S18 $\quad{ }^{31} \mathrm{P}$ NMR Spectra of Ni3/CsBAr ${ }_{4}+\mathrm{B}\left(\mathrm{C}_{6} \mathrm{~F}_{5}\right)_{3}$, RT $\quad$ S27

Table S13 Polymerization by Ni3-M + Post-Activation Time S28

\section{Electrochemical Studies}

$\begin{array}{lll}\text { Figure S19 Cyclic Voltammograms of Ni Complexes } & \text { S29 }\end{array}$

\section{Ni Complex Characterization}

Figure S20

${ }^{1} \mathrm{H}$ NMR Spectrum of $\mathbf{3}$

S30

Figure S21

${ }^{13} \mathrm{C}$ NMR Spectrum of 3

S31

Figure S22

${ }^{31} \mathrm{P}$ NMR Spectrum of $\mathbf{3}$

S32

Figure S23

${ }^{19}$ F NMR Spectrum of $\mathbf{3}$

S33

Figure S24

${ }^{1} \mathrm{H}$ NMR Spectrum of $\mathbf{N i 3}$

S34

Figure S25

${ }^{13} \mathrm{C}$ NMR Spectrum of Ni3

S35

Figure S26

Figure S27

${ }^{31} \mathrm{P}$ NMR Spectrum of Ni3

S36

$\begin{array}{ll}{ }^{19} \text { F NMR Spectrum of Ni3 } & \text { S37 }\end{array}$

\section{Polymer Characterization}

Figure S28

${ }^{1} \mathrm{H}$ NMR Spectrum of PE from Ni3-Li

S38

Figure S29

Figure S30

Figure S31

${ }^{1} \mathrm{H}$ NMR Spectrum of PE from Ni3-Na

S38

$\begin{array}{ll}{ }^{1} \mathrm{H} \text { NMR Spectrum of PE from Ni3-K } & \text { S39 }\end{array}$

Figure S32

${ }^{1} \mathrm{H}$ NMR Spectrum of PE from Ni3-Cs

S39

Figure S33

GPC Trace of PE from Ni3-Li

$\mathrm{S} 40$

Figure S34

GPC Trace of PE from Ni3-Na

$\mathrm{S} 40$

Figure S35

GPC Trace of PE from Ni3-K

S41

GPC Trace of PE from Ni3-Cs $\quad$ S41

X-ray Crystallographic Studies

Data Collection and Refinement

S42

Table S14

Crystal Data for Ni3-Li, Ni3-K and Ni3-Cs

S43

Figure S36

Figure S37

Molecular Structure of Ni3-Li

S44

$\begin{array}{ll}\text { Molecular Structure of Ni3-K } & \text { S45 }\end{array}$

Figure S38

Molecular Structure of Ni3-Cs

S46

S47

Additional Notes on Polymerization Studies

S48

Table S15

Cis and trans Isomer Distribution

S49

Catalyst Structure

Chart S1

DFT Optimized Structures of the Nickel Complexes

$\begin{array}{ll}\text { Depiction of Complexes Ni1 and Ni1' } & \text { S50 } \\ \text { S51- }\end{array}$

References

S52 


\section{Experimental}

A) Synthesis of complex Ni1<smiles>COCCOCOc1c(Br)cc(C)cc1P(c1ccccc1OC)c1ccccc1OC</smiles>

1

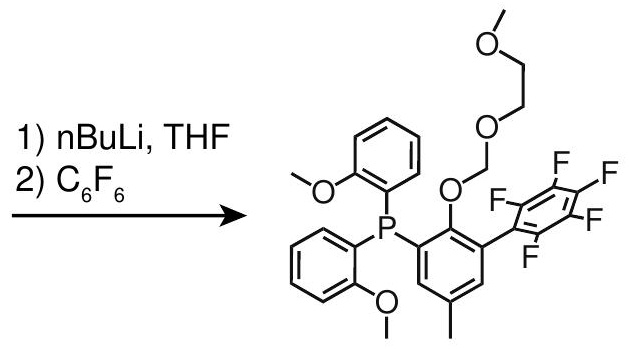

2

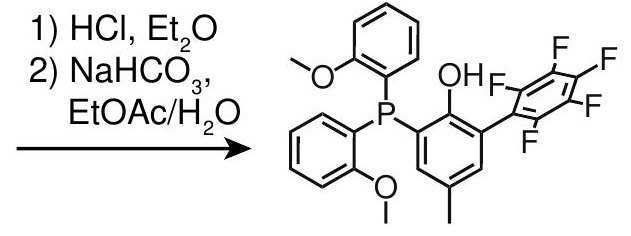

3
1) $\mathrm{NaH}, \mathrm{THF}$

2) $\mathrm{NiPhBr}\left(\mathrm{PMe}_{3}\right)_{2}$

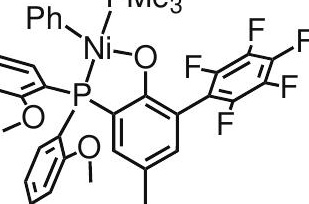

Ni1

B) Synthesis of complex $\mathbf{N i 2}$<smiles>COc1ccccc1P(c1ccccc1OC)c1cc(C)cc(C(C)(C)C)c1O</smiles>

4
1) $\mathrm{NaH}, \mathrm{THF}$ $\stackrel{\mathrm{NiPhBr}\left(\mathrm{PMe}_{3}\right)_{2}}{\longrightarrow}$

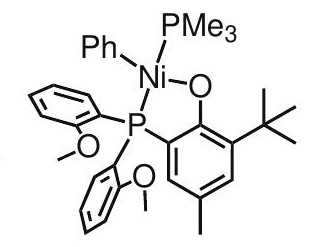

$\mathrm{Ni2}$

C) Synthesis of complex $\mathbf{N i 3}$<smiles>COCCOCCOCCOCc1cc(C)cc(P(c2ccccc2OC)c2ccccc2OC)c1O</smiles>

5
1) $\mathrm{NaH}, \mathrm{THF}$ 2) $\mathrm{NiPhBr}\left(\mathrm{PMe}_{3}\right)_{2}$

Scheme S1. Synthesis of complexes Ni1-Ni3. 


\section{Synthesis and Characterization}

Preparation of Compound 2. This synthesis was modified from a literature procedure. ${ }^{1}$ To a

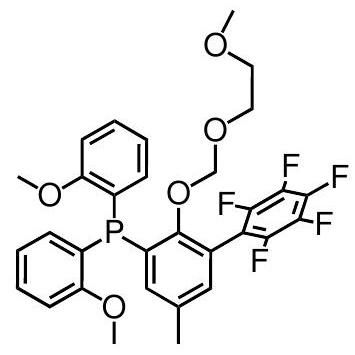
solution of compound 1 ( $4.64 \mathrm{~g}, 8.92 \mathrm{mmol}, 1.0$ equiv.) in $100 \mathrm{~mL}$ of dry THF in a $200 \mathrm{~mL}$ Schlenk flask under nitrogen at $-78^{\circ} \mathrm{C}, n \mathrm{BuLi}(1.6 \mathrm{M}$ in hexanes, $6.67 \mathrm{~mL}, 10.7 \mathrm{mmol}, 1.2$ equiv.) was added dropwise using a syringe pump. The reaction mixture was stirred at $-78{ }^{\circ} \mathrm{C}$ for $40 \mathrm{~min}$. Hexafluorobenzene (3.32 g, $2.1 \mathrm{~mL}, 17.87 \mathrm{mmol}, 2.0$ equiv.) was added dropwise via syringe. The resulting mixture was slowly warmed to room temperature and then allowed to stir overnight. The reaction was quenched by slow addition of $\mathrm{H}_{2} \mathrm{O}$ and the products were extracted into

$\mathrm{Et}_{2} \mathrm{O}(3 \times 100 \mathrm{~mL})$. The organic layers were combined, washed with $\mathrm{H}_{2} \mathrm{O}(2 \times 100 \mathrm{~mL})$, dried over $\mathrm{Na}_{2} \mathrm{SO}_{4}$, filtered, and evaporated to dryness. The crude material was purified by silica gel column chromatography $(3: 1$ hexane:ethyl acetate), followed by washing with pentane $(2 \times 10$ $\mathrm{mL})$ to afford a white solid $(3.1 \mathrm{~g}, 5.11 \mathrm{mmol}, 57 \%)$. This compound was used directly in the next step without further purification.

Preparation of Compound 3. This synthesis was modified from a literature procedure. ${ }^{1}$<smiles>COc1ccccc1P(c1ccccc1OC)c1cc(C)cc(C2(F)C(F)C(F)(F)C(F)(F)C2(F)F)c1O</smiles>
Compound 2 ( $3.1 \mathrm{~g}, 5.11 \mathrm{~mol}, 1.0$ equiv.) was dissolved in $50 \mathrm{~mL}$ of $\mathrm{MeOH}$ and then $150 \mathrm{~mL}$ solution of $2 \mathrm{M} \mathrm{HCl}$ in $\mathrm{Et}_{2} \mathrm{O}$ was added. The reaction mixture was stirred at room temperature overnight and then dried to remove solvent. The product was dissolved in $200 \mathrm{~mL}$ of EtOAc along and then combined with $50 \mathrm{~mL}$ of $1 \mathrm{M}$ aqueous $\mathrm{NaHCO}_{3}$. The mixture was stirred at room temperature for $30 \mathrm{~min}$ and the product was extracted into $\mathrm{Et}_{2} \mathrm{O}(3 \times 100 \mathrm{~mL})$. The organic layers were combined, washed with $\mathrm{H}_{2} \mathrm{O}(2 \times 100$ $\mathrm{mL}$ ), dried over $\mathrm{Na}_{2} \mathrm{SO}_{4}$, filtered, and evaporated to dryness. The crude material was purified by silica gel column chromatography (3:1 hexane:ethyl acetate). The resulting product was further recrystallized using a mixture of pentane and dichloromethane to afford a white solid $(1.9 \mathrm{~g}, 3.66$ mmol, 72\%). ${ }^{1} \mathrm{H}$ NMR (500 MHz, benzene- $\left.d_{6}\right): \delta 7.27-7.17(\mathrm{~m}, 3 \mathrm{H}), 7.07-6.99(\mathrm{~m}, 3 \mathrm{H}), 6.80$ $(\mathrm{s}, 1 \mathrm{H}), 6.70(\mathrm{t}, J=7.4 \mathrm{~Hz}, 2 \mathrm{H}), 6.36(\mathrm{dd}, J=8.1,5.0 \mathrm{~Hz}, 2 \mathrm{H}), 3.09(\mathrm{~s}, 6 \mathrm{H}), 1.82(\mathrm{~s}, 3 \mathrm{H}) .{ }^{13} \mathrm{C}$ NMR (126 MHz, Benzene- $\left.d_{6}\right): \delta 161.35,161.23,155.93,137.96,137.87,133.98,133.28$, $130.64,122.72,121.48,121.23,113.17,110.42,54.88,19.95 .{ }^{19} \mathrm{~F}$ NMR (470 MHz, benzene- $\left.d_{6}\right)$ : $\delta-140.43(\mathrm{dd}, J=24.7,6.9 \mathrm{~Hz}),-156.43(\mathrm{t}, J=21.5 \mathrm{~Hz}),-163.32--163.53(\mathrm{~m}) .{ }^{31} \mathrm{P}$ NMR $(202$ $\mathrm{MHz}$, Benzene- $\left.d_{6}\right): \delta-47.26$. ESI-MS(+) calc. for $\mathrm{C}_{27} \mathrm{H}_{20} \mathrm{~F}_{5} \mathrm{O}_{3} \mathrm{P}[\mathrm{M}+\mathrm{H}]^{+}=519.1142$, found 519.1133.

Preparation of Complex Ni1. Inside the glovebox, ligand 3 ( 0.193 g, 0.37 mmol, 1 equiv.) was

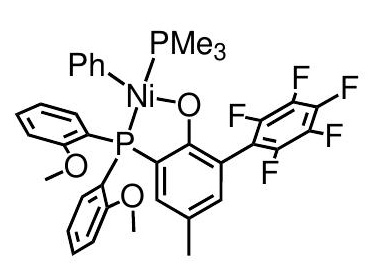
dissolved in $10 \mathrm{~mL}$ of THF. Small aliquots of $\mathrm{NaH}(60 \%, 0.3 \mathrm{~g}, 0.74$, 2.0 equiv.) were added and the mixture was stirred at room temperature for $2 \mathrm{~h}$. The solution was filtered to remove excess $\mathrm{NaH}$ and then combined with a solution of $\mathrm{NiPhBr}\left(\mathrm{PMe}_{3}\right)_{2}(0.121 \mathrm{~g}, 0.33 \mathrm{mmol}, 0.9$ equiv.) in $5 \mathrm{~mL}$ of benzene. The resulting mixture was stirred at room temperature overnight. The precipitate formed was removed by filtration and the filtrate was dried under vacuum. The crude material was dissolved in a mixture of $5 \mathrm{~mL}$ of pentane and $5 \mathrm{~mL}$ of toluene and the solution was filtered once again before evaporating to dryness. Finally, the solid was washed with pentane $(5 \times 3 \mathrm{~mL})$ and dried under vacuum to afford 
a yellow powder $(0.153 \mathrm{~g}, 0.21 \mathrm{mmol}, 64 \%) .{ }^{1} \mathrm{H}$ NMR $\left(500 \mathrm{MHz}\right.$, benzene- $\left.d_{6}\right): \delta 7.53-7.44(\mathrm{~m}$, $2 \mathrm{H}), 7.14(\mathrm{~d}, J=7.4 \mathrm{~Hz}, 2 \mathrm{H}), 7.09(\mathrm{~d}, J=11.1 \mathrm{~Hz}, 2 \mathrm{H}), 7.02(\mathrm{t}, J=7.8 \mathrm{~Hz}, 2 \mathrm{H}), 6.90(\mathrm{~s}, 1 \mathrm{H})$, $6.68(\mathrm{t}, J=7.4 \mathrm{~Hz}, 2 \mathrm{H}), 6.62(\mathrm{t}, J=7.4 \mathrm{~Hz}, 2 \mathrm{H}), 6.57(\mathrm{t}, J=7.1 \mathrm{~Hz}, 1 \mathrm{H}), 6.36(\mathrm{dd}, J=8.0,4.6$ $\mathrm{Hz}, 2 \mathrm{H}), 3.01(\mathrm{~s}, 6 \mathrm{H}), 1.91(\mathrm{~s}, 3 \mathrm{H}), 0.55(\mathrm{~d}, J=9.3 \mathrm{~Hz}, 9 \mathrm{H}) .{ }^{13} \mathrm{C}$ NMR $\left(101 \mathrm{MHz}\right.$, benzene- $\left.d_{6}\right): \delta$ $170.72,170.45,158.58,158.53,134.79,131.74,129.16,123.22,120.18,120.12,118.46,118.38$, $112.76,108.48,108.45,52.69,17.86,8.94,8.69 .{ }^{19} \mathrm{~F}$ NMR (470 MHz, benzene- $\left.d_{6}\right) \delta-138.98$ $(\mathrm{dd}, J=24.8,6.3 \mathrm{~Hz}),-159.39(\mathrm{t}, J=21.5 \mathrm{~Hz}),-165.02(\mathrm{td}, J=22.3,6.5 \mathrm{~Hz}) .{ }^{31} \mathrm{P}$ NMR $(202$ MHz, Benzene- $\left.d_{6}\right): \delta 10.57(\mathrm{~d}, J=288 \mathrm{~Hz}),-15.12(\mathrm{~d}, J=288 \mathrm{~Hz})$. Anal. Calcd for $\mathrm{C}_{36} \mathrm{H}_{33} \mathrm{~F}_{5} \mathrm{NiO}_{3} \mathrm{P}: \mathrm{C}, 59.29 ; \mathrm{H}, 4.56$. Found C, 59.39; H, 4.75. 


\section{Metal-Binding Studies}

UV-vis Absorption Spectroscopy: Metal Titration. Stock solutions of Ni3 and $\mathrm{MBAr}_{4}$ (or Li OTf) were prepared inside an inert nitrogen-filled glovebox. A $500 \mu \mathrm{M}$ stock solution of Ni3 were obtained by dissolving $25 \mu \mathrm{mol}$ of $\mathbf{N i 3}$ in $50 \mathrm{~mL}$ of $\mathrm{Et}_{2} \mathrm{O}$. A $10 \mathrm{~mL}$ aliquot of this $500 \mu \mathrm{M}$ solution was diluted to $50 \mathrm{~mL}$ using a volumetric flask to give a final concentration of $100 \mu \mathrm{M}$. The $3.0 \mathrm{mM}$ stock solution of $\mathrm{MBAr}_{4}{ }_{4}$ was obtained by dissolving $30 \mu \mathrm{mol}$ of $\mathrm{MBAr}_{4} \mathrm{~F}_{4}$ in $10 \mathrm{~mL}$ of $\mathrm{Et}_{2} \mathrm{O}$ using a volumetric flask. A $3.0 \mathrm{~mL}$ solution of $\mathrm{Ni3}$ was transferred to a $1 \mathrm{~cm}$ quartz cuvette and then sealed with a septum screw cap. A $100 \mu \mathrm{L}$ airtight syringe was loaded with the $3.0 \mathrm{mM}$ solution of $\mathrm{MBAr}^{\mathrm{F}}$. The cuvette was placed inside a UV-vis spectrophotometer and the spectrum of the Ni3 solution was recorded. Aliquots containing 0.1 equiv. of $\mathrm{MBAr}_{4}(10 \mu \mathrm{L})$, relative to the nickel complex, were added and the solution was allowed to reach equilibrium before the spectra were measured (about 20-30 min). The titration experiments were stopped after the addition of up to 1.0 equiv. of $\mathrm{MBAr}^{\mathrm{F}}$. 


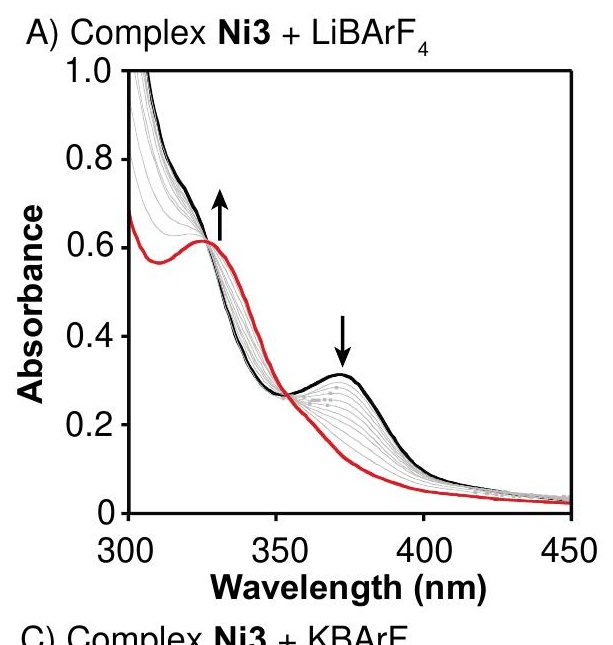

B) Complex Ni3 $+\mathrm{NaBArF}_{4}$
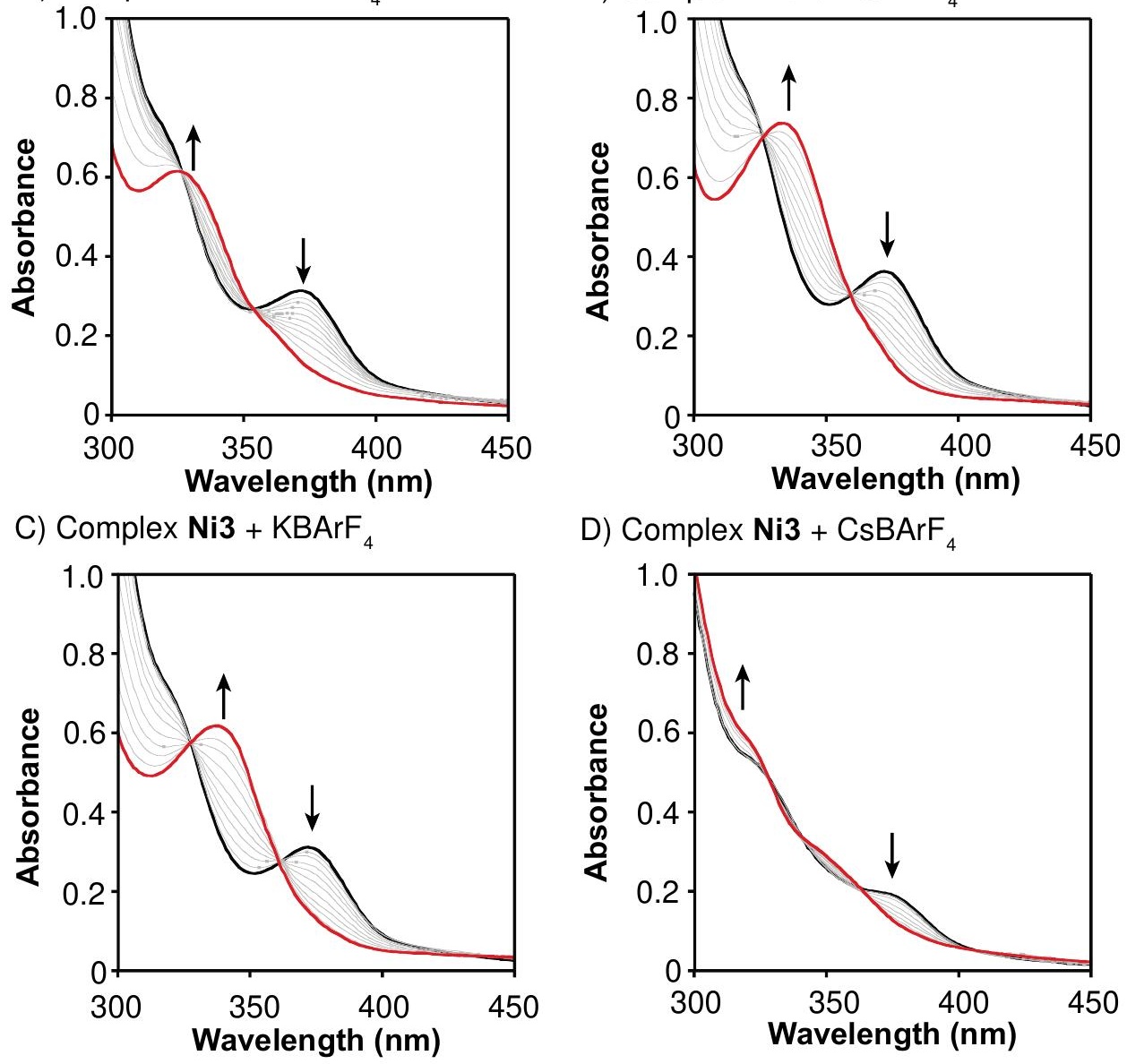

D) Complex $\mathrm{Ni3}+\mathrm{CsBArF}_{4}$

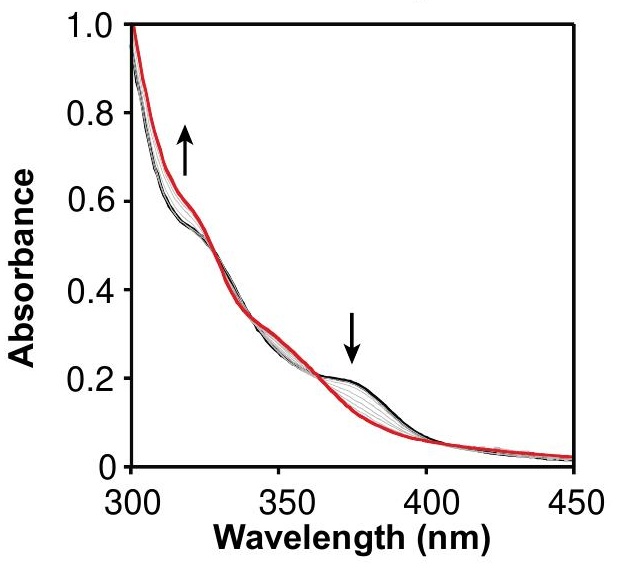

E) Complex Ni3 + LiOTf

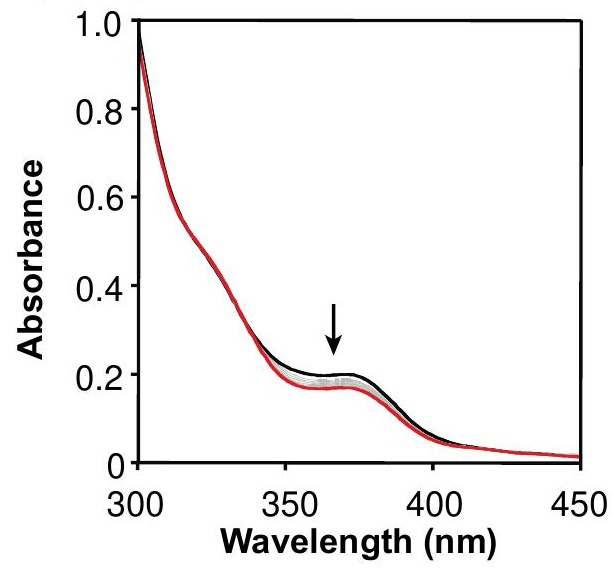

Figure S1. UV-vis absorbance spectra of complex Ni3 $\left(100 \mu \mathrm{M}\right.$ in $\left.\mathrm{Et}_{2} \mathrm{O}\right)$ after the addition of up to 1.0 equiv. of A) $\mathrm{LiBAr}_{4}$, B) $\mathrm{NaBAr}_{4}$, C) $\mathrm{KBAr}_{4}$, D) $\mathrm{CsBAr}_{4}$, and E) LiOTf. The starting trace of $\mathrm{Ni3}$ is shown in black and the final trace (+ 1.0 equiv. of $\mathrm{Li}^{+}$relative to $\left.\mathrm{Ni}\right)$ is shown in red. 
UV-Vis Absorption Spectroscopy: Job Plot Studies. Stock solutions of Ni3 (500 $\mu \mathrm{M})$ and $\mathrm{MBAr}_{4}(500 \mu \mathrm{M})(\mathrm{M}=\mathrm{Li}, \mathrm{Na}, \mathrm{K}$ and $\mathrm{Cs})$ in $\mathrm{Et}_{2} \mathrm{O}$ were prepared in separate volumetric flasks inside the drybox. Stock solutions of $\mathbf{N i 3}$ and $\mathrm{MBAr}_{4}{ }_{4}$ were combined in different ratios to give 10 different samples, each having a final volume of $3.0 \mathrm{~mL}$. The samples were recorded by UVvis absorption spectroscopy at RT.

The UV-vis spectral data were analyzed according to the method reported by Hirose. ${ }^{2}$ In our case, the host $(\mathrm{H})$ is $\mathbf{N i 3}$, the guest $(\mathrm{g})$ is $\mathrm{M}^{+}$, and the complex $(\mathrm{C})$ is Ni3-M. Since the alkali salt has no absorption in the $300-500 \mathrm{~nm}$ range, we used this simplified expression to analyze the data: $A_{\mathrm{obs}}-\varepsilon_{\mathrm{h}} \cdot[\mathrm{H}]_{\mathrm{t}}=\left(\varepsilon_{\mathrm{C}}-\mathrm{a} \cdot \varepsilon_{\mathrm{h}}\right) \cdot[\mathrm{C}]$, where $\mathrm{A}_{\mathrm{obs}}=$ observed absorbance, $\mathrm{a}=$ constant, $\varepsilon_{\mathrm{h}}=$ molar absorptivity of host $\mathbf{N i 3}, \varepsilon_{\mathrm{C}}=$ molar absorptivity of $\mathrm{Ni3}-\mathrm{M},[\mathrm{H}]_{\mathrm{t}}{ }^{=}$starting concentration of host Ni3, and $[\mathrm{C}]=$ observed concentration of Ni3-M. Since $[\mathrm{C}]$ is proportional to $\mathrm{A}_{\mathrm{obs}}-\varepsilon_{\mathrm{h}} \cdot[\mathrm{H}]_{\mathrm{t}}$, a Job Plot was constructed by plotting $\mathrm{A}_{\mathrm{obs}}-\varepsilon_{\mathrm{h}} \cdot[\mathrm{H}]_{\mathrm{t}}$ vs. $\chi_{\mathrm{Ni}}$ (the mole ratio of $\mathbf{N i 3}=$ $[\mathrm{Ni3}] /([\mathrm{Ni3}]+[\mathrm{M}]))$. 
Table S1. Job Plot Data and Calculations for Ni3-Li ${ }^{a}$

\begin{tabular}{ccccccc}
\hline$\chi \mathrm{Ni}$ & $\begin{array}{c}\text { Volume of } \\
\text { Stock } \\
\text { Soln of H } \\
(\mathrm{mL})\end{array}$ & $\begin{array}{c}\text { Amount of } \\
\text { H Added } \\
(\mathrm{mol})\end{array}$ & $\begin{array}{c}\text { Final Conc. } \\
\text { of H } \\
(\mathbf{M})\end{array}$ & $\begin{array}{c}\mathbf{A h}_{\mathbf{h}} \\
\text { (calculated) }\end{array}$ & $\begin{array}{c}\text { Aobs } \\
\text { (@379 nm) }\end{array}$ & $\mathbf{A}_{\mathbf{h}-\mathbf{A}_{\text {obs }}}$ \\
\hline 1.0 & $3.000 \mathrm{E}-03$ & $1.500 \mathrm{E}-06$ & $5.000 \mathrm{E}-04$ & $1.482 \mathrm{E}+00$ & $1.482 \mathrm{E}+00$ & $0.000 \mathrm{E}+00$ \\
0.9 & $2.700 \mathrm{E}-03$ & $1.350 \mathrm{E}-06$ & $4.500 \mathrm{E}-04$ & $1.334 \mathrm{E}+00$ & $1.295 \mathrm{E}+00$ & $3.873 \mathrm{E}-02$ \\
0.8 & $2.400 \mathrm{E}-03$ & $1.200 \mathrm{E}-06$ & $4.000 \mathrm{E}-04$ & $1.186 \mathrm{E}+00$ & $1.028 \mathrm{E}+00$ & $1.582 \mathrm{E}-01$ \\
0.7 & $2.100 \mathrm{E}-03$ & $1.050 \mathrm{E}-06$ & $3.500 \mathrm{E}-04$ & $1.038 \mathrm{E}+00$ & $7.182 \mathrm{E}-01$ & $3.195 \mathrm{E}-01$ \\
0.6 & $1.800 \mathrm{E}-03$ & $9.000 \mathrm{E}-07$ & $3.000 \mathrm{E}-04$ & $8.894 \mathrm{E}-01$ & $4.922 \mathrm{E}-01$ & $3.972 \mathrm{E}-01$ \\
0.5 & $1.500 \mathrm{E}-03$ & $7.500 \mathrm{E}-07$ & $2.500 \mathrm{E}-04$ & $7.412 \mathrm{E}-01$ & $2.411 \mathrm{E}-01$ & $5.001 \mathrm{E}-01$ \\
0.4 & $1.200 \mathrm{E}-03$ & $6.000 \mathrm{E}-07$ & $2.000 \mathrm{E}-04$ & $5.929 \mathrm{E}-01$ & $1.442 \mathrm{E}-01$ & $4.488 \mathrm{E}-01$ \\
0.3 & $9.000 \mathrm{E}-04$ & $4.500 \mathrm{E}-07$ & $1.500 \mathrm{E}-04$ & $4.447 \mathrm{E}-01$ & $1.031 \mathrm{E}-01$ & $3.416 \mathrm{E}-01$ \\
0.2 & $6.000 \mathrm{E}-04$ & $3.000 \mathrm{E}-07$ & $1.000 \mathrm{E}-04$ & $2.965 \mathrm{E}-01$ & $6.749 \mathrm{E}-02$ & $2.290 \mathrm{E}-01$ \\
0.1 & $3.000 \mathrm{E}-04$ & $1.500 \mathrm{E}-07$ & $5.000 \mathrm{E}-05$ & $1.482 \mathrm{E}-01$ & $3.717 \mathrm{E}-02$ & $1.111 \mathrm{E}-01$ \\
0.0 & $0.000 \mathrm{E}+00$ & $0.000 \mathrm{E}+00$ & $0.000 \mathrm{E}+00$ & $0.000 \mathrm{E}+00$ & $-4.366 \mathrm{E}-03$ & $4.366 \mathrm{E}-03$
\end{tabular}

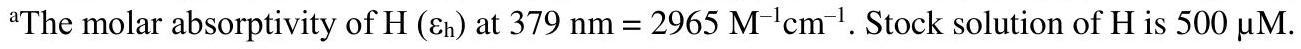

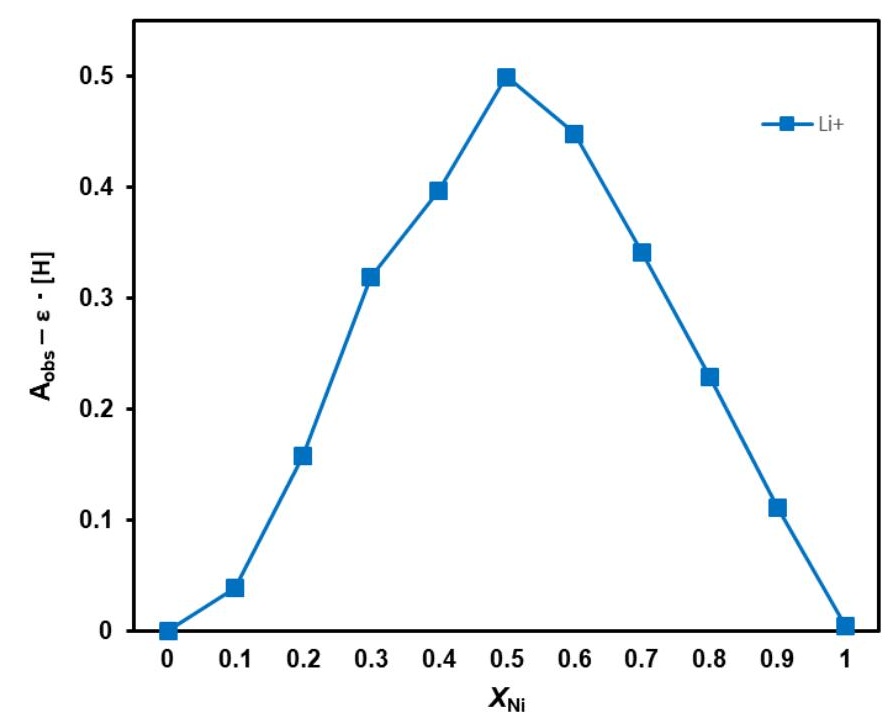

Figure S2. Job Plot showing the coordination interactions between complex Ni3 and $\operatorname{LiBAr}_{4}$. The peak maximum occurs at $\chi_{\mathrm{Ni}}=0.5$, which suggests that the optimal nickel:lithium binding stoichiometry is $1: 1$. The $y$-axis value $\left(\mathrm{A}_{\mathrm{obs}}-\varepsilon_{\mathrm{h}} \cdot[\mathrm{H}]_{\mathrm{t}}\right)$ is proportional to the concentration of the nickel-sodium complex Ni3-Li. The $x$-axis is the molar ratio of nickel $\left(\chi_{\mathrm{Ni}}=\right.$ $\left.[\mathbf{N i 3}] /\left([\mathrm{Ni3}]+\left[\mathrm{Li}^{+}\right]\right)\right)$. 
Table S2. Job Plot Data and Calculations Used for Ni3-Na ${ }^{a}$

\begin{tabular}{ccccccc}
\hline$\chi \mathrm{Ni}$ & $\begin{array}{c}\text { Volume of } \\
\text { Stock } \\
\text { Soln of H } \\
(\mathrm{mL})\end{array}$ & $\begin{array}{c}\text { Amount of } \\
\text { H Added } \\
(\mathrm{mol})\end{array}$ & $\begin{array}{c}\text { Final Conc. } \\
\text { of H } \\
(\mathbf{M})\end{array}$ & $\begin{array}{c}\mathbf{A h}_{\mathbf{h}} \\
\text { (calculated) }\end{array}$ & $\begin{array}{c}\text { Aobs } \\
\text { (@330 nm) }\end{array}$ & Aobs-Ah \\
\hline 1.0 & $3.000 \mathrm{E}-03$ & $1.500 \mathrm{E}-06$ & $5.000 \mathrm{E}-04$ & $1.238 \mathrm{E}+00$ & $1.238 \mathrm{E}+00$ & $0.000 \mathrm{E}+00$ \\
0.9 & $2.700 \mathrm{E}-03$ & $1.350 \mathrm{E}-06$ & $4.500 \mathrm{E}-04$ & $1.115 \mathrm{E}+00$ & $1.059 \mathrm{E}+00$ & $5.543 \mathrm{E}-02$ \\
0.8 & $2.400 \mathrm{E}-03$ & $1.200 \mathrm{E}-06$ & $4.000 \mathrm{E}-04$ & $9.908 \mathrm{E}-01$ & $8.340 \mathrm{E}-01$ & $1.568 \mathrm{E}-01$ \\
0.7 & $2.100 \mathrm{E}-03$ & $1.050 \mathrm{E}-06$ & $3.500 \mathrm{E}-04$ & $8.669 \mathrm{E}-01$ & $5.645 \mathrm{E}-01$ & $3.024 \mathrm{E}-01$ \\
0.6 & $1.800 \mathrm{E}-03$ & $9.000 \mathrm{E}-07$ & $3.000 \mathrm{E}-04$ & $7.431 \mathrm{E}-01$ & $3.553 \mathrm{E}-01$ & $3.877 \mathrm{E}-01$ \\
0.5 & $1.500 \mathrm{E}-03$ & $7.500 \mathrm{E}-07$ & $2.500 \mathrm{E}-04$ & $6.192 \mathrm{E}-01$ & $1.951 \mathrm{E}-01$ & $4.241 \mathrm{E}-01$ \\
0.4 & $1.200 \mathrm{E}-03$ & $6.000 \mathrm{E}-07$ & $2.000 \mathrm{E}-04$ & $4.954 \mathrm{E}-01$ & $1.578 \mathrm{E}-01$ & $3.376 \mathrm{E}-01$ \\
0.3 & $9.000 \mathrm{E}-04$ & $4.500 \mathrm{E}-07$ & $1.500 \mathrm{E}-04$ & $3.715 \mathrm{E}-01$ & $1.149 \mathrm{E}-01$ & $2.567 \mathrm{E}-01$ \\
0.2 & $6.000 \mathrm{E}-04$ & $3.000 \mathrm{E}-07$ & $1.000 \mathrm{E}-04$ & $2.477 \mathrm{E}-01$ & $7.796 \mathrm{E}-02$ & $1.697 \mathrm{E}-01$ \\
0.1 & $3.000 \mathrm{E}-04$ & $1.500 \mathrm{E}-07$ & $5.000 \mathrm{E}-05$ & $1.238 \mathrm{E}-01$ & $4.617 \mathrm{E}-02$ & $7.768 \mathrm{E}-02$ \\
0.0 & $0.000 \mathrm{E}+00$ & $0.000 \mathrm{E}+00$ & $0.000 \mathrm{E}+00$ & $0.000 \mathrm{E}+00$ & $0.000 \mathrm{E}+00$ & $0.000 \mathrm{E}+00$
\end{tabular}

${ }^{a}$ The molar absorptivity of $\mathrm{H}\left(\varepsilon_{\mathrm{h}}\right)$ at $379 \mathrm{~nm}=2477 \mathrm{M}^{-1} \mathrm{~cm}^{-1}$. Stock solution of $\mathrm{H}$ is $500 \mu \mathrm{M}$.

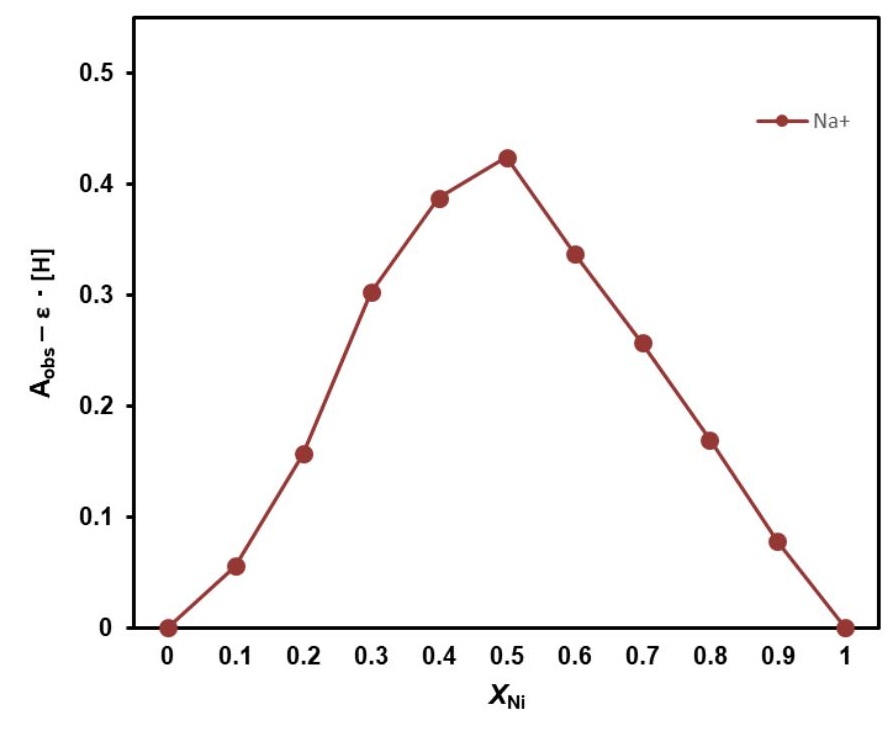

Figure S3. Job Plot showing the coordination interactions between complex Ni3 and $\mathrm{NaBAr}_{4}$. The peak maximum occurs at $\chi_{\mathrm{Ni}}=0.5$, which suggests that the optimal nickel:sodium binding stoichiometry is $1: 1$. The $y$-axis value $\left(\mathrm{A}_{\mathrm{obs}}-\varepsilon_{\mathrm{h}} \cdot[\mathrm{H}]_{\mathrm{t}}\right)$ is proportional to the concentration of the nickel-sodium complex Ni3-Na. The $x$-axis is the molar ratio of nickel $\left(\chi_{\mathrm{Ni}}=\right.$ $\left.[\mathbf{N i 3}] /\left([\mathrm{Ni3}]+\left[\mathrm{Na}^{+}\right]\right)\right)$. 
Table S3. Job Plot Data and Calculations Used for Ni3-K ${ }^{a}$

\begin{tabular}{ccccccc}
\hline$\chi \mathbf{N i}$ & $\begin{array}{c}\text { Volume of } \\
\text { Stock } \\
\text { Soln of H } \\
(\mathrm{mL})\end{array}$ & $\begin{array}{c}\text { Amount of } \\
\text { H Added } \\
(\mathrm{mol})\end{array}$ & $\begin{array}{c}\text { Final Conc. } \\
\text { of H } \\
(\mathrm{M})\end{array}$ & $\begin{array}{c}\mathbf{A h}_{\mathbf{h}} \\
(\text { calculated })\end{array}$ & $\begin{array}{c}\text { Aobs } \\
(@ 330 \mathrm{~nm})\end{array}$ & $\mathbf{A}_{\text {obs-A }}$ \\
\hline 1.0 & $3.000 \mathrm{E}-03$ & $1.500 \mathrm{E}-06$ & $5.000 \mathrm{E}-04$ & $1.201 \mathrm{E}+00$ & $1.201 \mathrm{E}+00$ & $0.000 \mathrm{E}+00$ \\
0.9 & $2.700 \mathrm{E}-03$ & $1.350 \mathrm{E}-06$ & $4.500 \mathrm{E}-04$ & $1.081 \mathrm{E}+00$ & $1.073 \mathrm{E}+00$ & $7.443 \mathrm{E}-03$ \\
0.8 & $2.400 \mathrm{E}-03$ & $1.200 \mathrm{E}-06$ & $4.000 \mathrm{E}-04$ & $9.606 \mathrm{E}-01$ & $8.741 \mathrm{E}-01$ & $8.649 \mathrm{E}-02$ \\
0.7 & $2.100 \mathrm{E}-03$ & $1.050 \mathrm{E}-06$ & $3.500 \mathrm{E}-04$ & $8.406 \mathrm{E}-01$ & $6.233 \mathrm{E}-01$ & $2.172 \mathrm{E}-01$ \\
0.6 & $1.800 \mathrm{E}-03$ & $9.000 \mathrm{E}-07$ & $3.000 \mathrm{E}-04$ & $7.205 \mathrm{E}-01$ & $4.245 \mathrm{E}-01$ & $2.959 \mathrm{E}-01$ \\
0.5 & $1.500 \mathrm{E}-03$ & $7.500 \mathrm{E}-07$ & $2.500 \mathrm{E}-04$ & $6.004 \mathrm{E}-01$ & $2.225 \mathrm{E}-01$ & $3.779 \mathrm{E}-01$ \\
0.4 & $1.200 \mathrm{E}-03$ & $6.000 \mathrm{E}-07$ & $2.000 \mathrm{E}-04$ & $4.803 \mathrm{E}-01$ & $1.635 \mathrm{E}-01$ & $3.168 \mathrm{E}-01$ \\
0.3 & $9.000 \mathrm{E}-04$ & $4.500 \mathrm{E}-07$ & $1.500 \mathrm{E}-04$ & $3.602 \mathrm{E}-01$ & $1.167 \mathrm{E}-01$ & $2.435 \mathrm{E}-01$ \\
0.2 & $6.000 \mathrm{E}-04$ & $3.000 \mathrm{E}-07$ & $1.000 \mathrm{E}-04$ & $2.402 \mathrm{E}-01$ & $8.181 \mathrm{E}-02$ & $1.583 \mathrm{E}-01$ \\
0.1 & $3.000 \mathrm{E}-04$ & $1.500 \mathrm{E}-07$ & $5.000 \mathrm{E}-05$ & $1.201 \mathrm{E}-01$ & $4.852 \mathrm{E}-02$ & $7.156 \mathrm{E}-02$ \\
0.0 & $0.000 \mathrm{E}+00$ & $0.000 \mathrm{E}+00$ & $0.000 \mathrm{E}+00$ & $0.000 \mathrm{E}+00$ & $0.000 \mathrm{E}+00$ & $0.000 \mathrm{E}+00$
\end{tabular}

${ }^{a}$ The molar absorptivity of $\mathrm{H}\left(\varepsilon_{\mathrm{h}}\right)$ at $379 \mathrm{~nm}=2402 \mathrm{M}^{-1} \mathrm{~cm}^{-1}$. Stock solution of $\mathrm{H}$ is $500 \mu \mathrm{M}$.

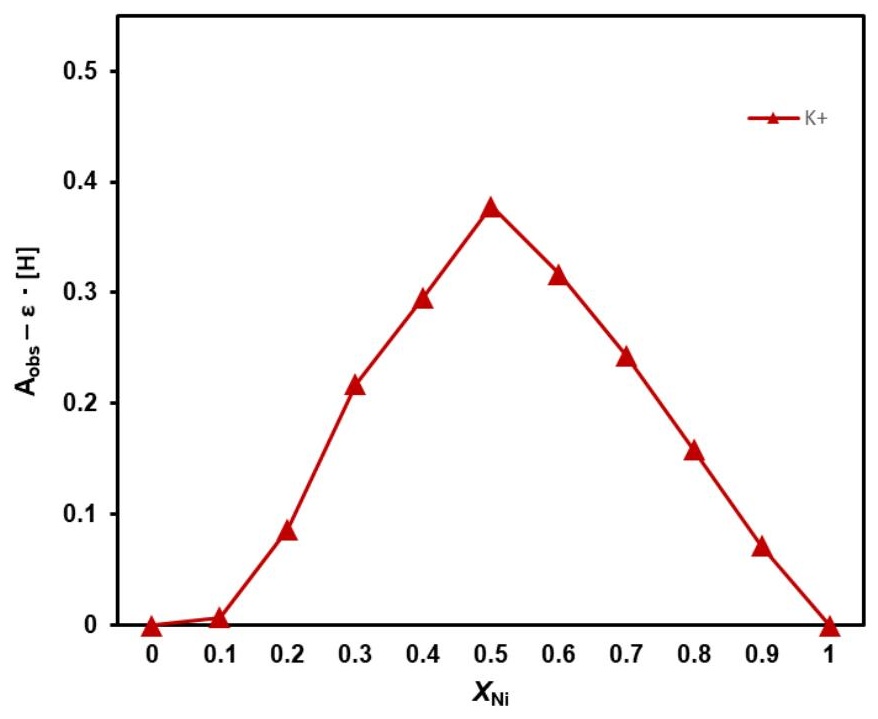

Figure S4. Job Plot showing the coordination interactions between complex Ni3 and $\operatorname{KBAr}_{4}$. The peak maximum occurs at $\chi_{\mathrm{Ni}}=0.5$, which suggests that the optimal nickel:potassium binding stoichiometry is $1: 1$. The $y$-axis value $\left(\mathrm{A}_{\mathrm{obs}}-\varepsilon_{\mathrm{h}} \cdot[\mathrm{H}]_{\mathrm{t}}\right)$ is proportional to the concentration of the nickel-sodium complex Ni3-K. The $\mathrm{x}$-axis is the molar ratio of nickel $\left(\chi_{\mathrm{Ni}}=[\mathbf{N i 3}] /\left([\mathrm{Ni3}]+\left[\mathrm{K}^{+}\right]\right)\right)$. 
Table S4. Job Plot Data and Calculations Used for Ni3-Cs ${ }^{a}$

\begin{tabular}{ccccccc}
\hline $\boldsymbol{\chi N}$ Ni & $\begin{array}{c}\text { Volume of } \\
\text { Stock Soln } \\
\text { of H } \\
(\mathrm{mL})\end{array}$ & $\begin{array}{c}\text { Amount of } \\
\text { H Added } \\
(\mathrm{mol})\end{array}$ & $\begin{array}{c}\text { Final Conc. } \\
\text { of H } \\
(\mathrm{M})\end{array}$ & $\begin{array}{c}\text { Ah } \\
(\text { calculated })\end{array}$ & $\begin{array}{c}\text { Aobs } \\
(@ 330 \mathrm{~nm})\end{array}$ & $\mathbf{A}_{\text {obs-Ah }}$ \\
\hline 1.0 & $3.000 \mathrm{E}-03$ & $1.500 \mathrm{E}-06$ & $5.000 \mathrm{E}-04$ & $1.248 \mathrm{E}+00$ & $1.25 \mathrm{E}+00$ & $0.00 \mathrm{E}+00$ \\
0.9 & $2.700 \mathrm{E}-03$ & $1.350 \mathrm{E}-06$ & $4.500 \mathrm{E}-04$ & $1.123 \mathrm{E}+00$ & 1.076849341 & 0.046550346 \\
0.8 & $2.400 \mathrm{E}-03$ & $1.200 \mathrm{E}-06$ & $4.000 \mathrm{E}-04$ & $9.986 \mathrm{E}-01$ & 0.89023757 & 0.108339929 \\
0.7 & $2.100 \mathrm{E}-03$ & $1.050 \mathrm{E}-06$ & $3.500 \mathrm{E}-04$ & $8.738 \mathrm{E}-01$ & 0.732032239 & 0.141723072 \\
0.6 & $1.800 \mathrm{E}-03$ & $9.000 \mathrm{E}-07$ & $3.000 \mathrm{E}-04$ & $7.489 \mathrm{E}-01$ & 0.545079529 & 0.203853595 \\
0.5 & $1.500 \mathrm{E}-03$ & $7.500 \mathrm{E}-07$ & $2.500 \mathrm{E}-04$ & $6.241 \mathrm{E}-01$ & 0.382887274 & 0.241223663 \\
0.4 & $1.200 \mathrm{E}-03$ & $6.000 \mathrm{E}-07$ & $2.000 \mathrm{E}-04$ & $4.993 \mathrm{E}-01$ & 0.301692158 & 0.197596592 \\
0.3 & $9.000 \mathrm{E}-04$ & $4.500 \mathrm{E}-07$ & $1.500 \mathrm{E}-04$ & $3.745 \mathrm{E}-01$ & 0.24214679 & 0.132319772 \\
0.2 & $6.000 \mathrm{E}-04$ & $3.000 \mathrm{E}-07$ & $1.000 \mathrm{E}-04$ & $2.496 \mathrm{E}-01$ & 0.155732036 & 0.093912339 \\
0.1 & $3.000 \mathrm{E}-04$ & $1.500 \mathrm{E}-07$ & $5.000 \mathrm{E}-05$ & $1.248 \mathrm{E}-01$ & 0.089259788 & 0.035562399 \\
0.0 & $0.000 \mathrm{E}+00$ & $0.000 \mathrm{E}+00$ & $0.000 \mathrm{E}+00$ & $0.000 \mathrm{E}+00$ & 0.023227019 & 0
\end{tabular}

aThe molar absorptivity of $\mathrm{H}\left(\varepsilon_{\mathrm{h}}\right)$ at $379 \mathrm{~nm}=2500 \mathrm{M}^{-1} \mathrm{~cm}^{-1}$. Stock solution of $\mathrm{H}$ is $500 \mu \mathrm{M}$.

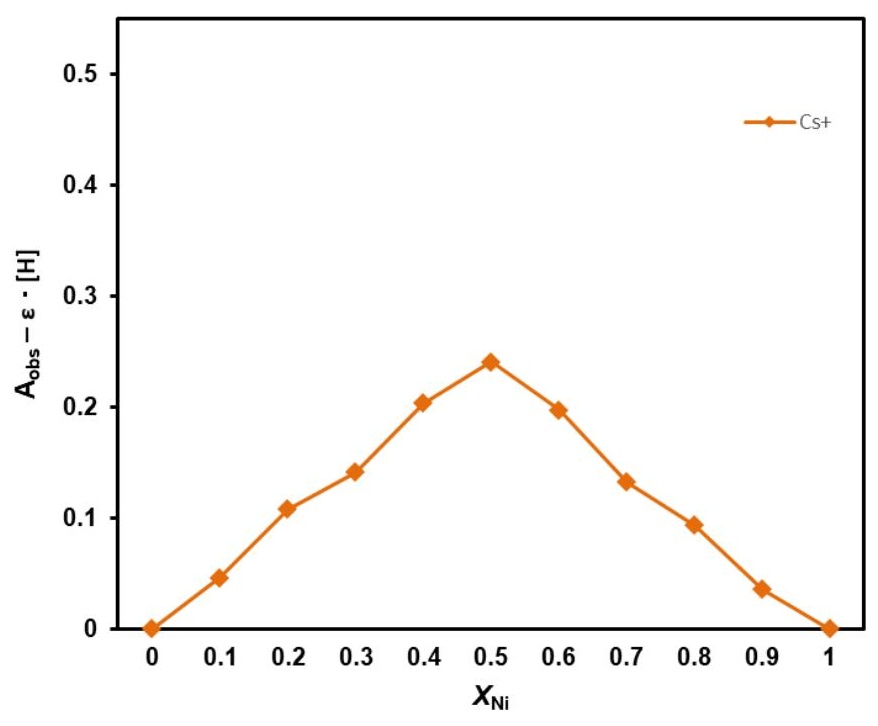

Figure S5. Job Plot showing the coordination interactions between complex Ni3 and $\mathrm{CsBAr}_{4}$. The peak maximum occurs at $\chi_{\mathrm{Ni}}=0.5$, which suggests that the optimal nickel:cesium binding stoichiometry is $1: 1$. The $y$-axis value $\left(\mathrm{A}_{\mathrm{obs}}-\varepsilon_{\mathrm{h}} \cdot[\mathrm{H}]_{\mathrm{t}}\right)$ is proportional to the concentration of the nickel-cesium complex Ni3-Cs. The $\mathrm{x}$-axis is the molar ratio of nickel $\left(\chi_{\mathrm{Ni}}=\right.$ $\left.[\mathbf{N i 3}] /\left([\mathrm{Ni3}]+\left[\mathrm{Cs}^{+}\right]\right)\right)$. 


\section{Polymerization Studies}

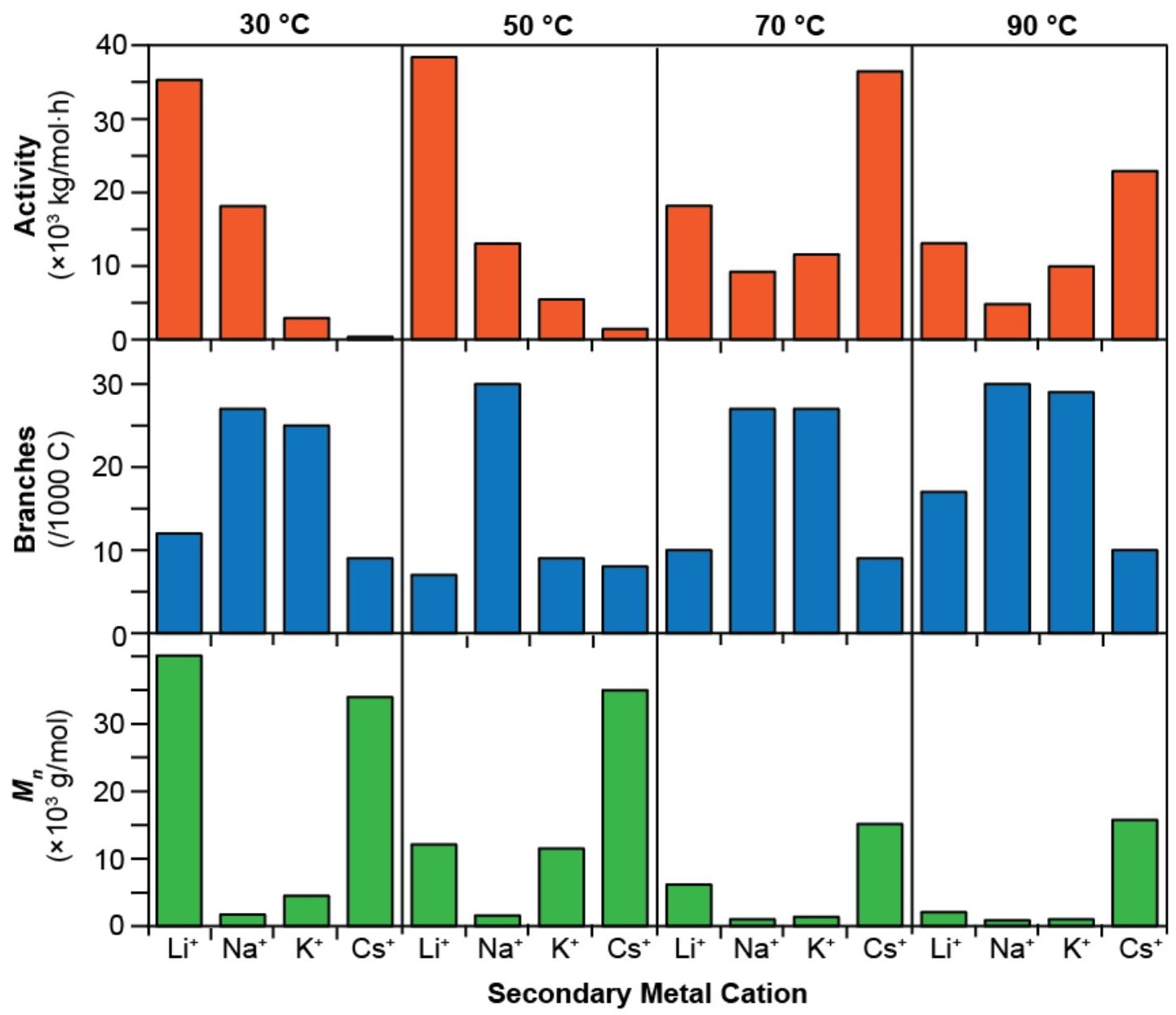

Figure S6. Effect of temperature on polymerization activity of Ni3 and its polymer branching and molecular weight. Full data shown in Table 2 
Table S5. Ethylene Polymerization Catalyzed by Ni3 and Various Salts in Different Solvents ${ }^{a}$

\begin{tabular}{|c|c|c|c|c|c|c|}
\hline Entry & $\begin{array}{c}\text { Salt } \\
\text { (equiv.) }\end{array}$ & Solvent & $\begin{array}{l}\text { Polymer } \\
\text { Yield (g) }\end{array}$ & $\begin{array}{c}\text { Activity } \\
(\mathrm{kg} / \mathrm{mol} \cdot \mathrm{h})\end{array}$ & $\begin{array}{c}\boldsymbol{M}^{b} \\
\left(\times 0^{3}\right)\end{array}$ & $\boldsymbol{M}_{\boldsymbol{w}} / \boldsymbol{M}_{\boldsymbol{n}}^{b}$ \\
\hline 1 & $\mathrm{Li}^{+}(2)$ & benzene & 4.3 & 43000 & 35.76 & 1.3 \\
\hline 2 & $\mathrm{Na}^{+}(2)$ & benzene & 8.4 & 17000 & 1.90 & 1.4 \\
\hline 3 & $\mathrm{~K}^{+}(2)$ & benzene & 7.2 & 14000 & 6.06 & 1.6 \\
\hline 4 & $\mathrm{Cs}^{+}(2)$ & benzene & 0.13 & 260 & 48.17 & 1.3 \\
\hline 5 & $\mathrm{Li}^{+}(2)$ & $\mathrm{CH}_{2} \mathrm{Cl}_{2}$ & 1.53 & 3100 & 39.30 & 1.3 \\
\hline 6 & $\mathrm{Na}^{+}(2)$ & $\mathrm{CH}_{2} \mathrm{Cl}_{2}$ & 0.25 & 500 & 1.06 & 1.4 \\
\hline 7 & $\mathrm{~K}^{+}(2)$ & $\mathrm{CH}_{2} \mathrm{Cl}_{2}$ & 0.61 & 1200 & 5.86 & 1.3 \\
\hline 8 & $\mathrm{Cs}^{+}(2)$ & $\mathrm{CH}_{2} \mathrm{Cl}_{2}$ & 0.22 & 440 & 15.99 & 1.3 \\
\hline $9^{c}$ & $\mathrm{~K}^{+}(2)$ & $\begin{array}{c}\mathrm{Et}_{2} \mathrm{O} / \text { toluene } \\
(1: 9)\end{array}$ & 8.1 & 3200 & 16.70 & 1.5 \\
\hline $10^{c}$ & $\mathrm{~K}^{+}(8)$ & $\begin{array}{c}\mathrm{Et}_{2} \mathrm{O} / \text { toluene } \\
(1: 9)\end{array}$ & 26.76 & 11000 & 5.60 & 1.6 \\
\hline $11^{c}$ & $\mathrm{~K}^{+}(8)$ & $\begin{array}{c}\text { THF/toluene } \\
(1: 9)\end{array}$ & 1.55 & 620 & 13.60 & 1.3 \\
\hline $12^{c}$ & $\mathrm{Na}^{+}(8)$ & $\begin{array}{c}\text { THF/toluene } \\
(1: 9)\end{array}$ & 6.44 & 2600 & 2.40 & 1.4 \\
\hline $13^{c}$ & $\mathrm{Na}^{+}(30)$ & $\begin{array}{c}\text { THF/toluene } \\
(1: 9)\end{array}$ & 13.22 & 5300 & 1.57 & 1.3 \\
\hline $14^{c}$ & $\mathrm{Na}^{+}(30)$ & $\begin{array}{c}\text { THF/toluene } \\
(2: 8)\end{array}$ & 0.32 & 130 & 5.10 & 1.4 \\
\hline $15^{c}$ & $\mathrm{Li}^{+}(30)$ & $\begin{array}{c}\text { THF/toluene } \\
(2: 8)\end{array}$ & 1.20 & 480 & 11.10 & 1.4 \\
\hline
\end{tabular}

${ }^{a}$ Polymerization conditions: Ni3 $(0.5 \mu \mathrm{mol}), \mathrm{MBAr}_{4}(1 \mu \mathrm{mol}), \mathrm{Ni}(\mathrm{COD}){ }_{2}(4 \mu \mathrm{mol})$, ethylene (450 psi), solvent (100 $\mathrm{mL}), 30^{\circ} \mathrm{C}, 1 \mathrm{~h}$. Temperature was controlled by manual external cooling when necessary to ensure that the reaction temperature does not exceed greater than $5^{\circ} \mathrm{C}$ from the starting temperature. Reported yields are the average of 2-3 runs and standard deviations are less than $10 \%$. ${ }^{b}$ Determined by GPC in trichlorobenzene at $140{ }^{\circ} \mathrm{C}$. ${ }^{c}$ Polymerization conditions: Ni3 $(5 \mu \mathrm{mol}), \mathrm{Ni}[\mathrm{COD}]_{2}(20 \mu \mathrm{mol})$, ethylene $(150 \mathrm{psi}), 100 \mathrm{~mL}$ solvent, $1 \mathrm{~h}, 50{ }^{\circ} \mathrm{C}$. Temperature was not controlled by manual external cooling. 


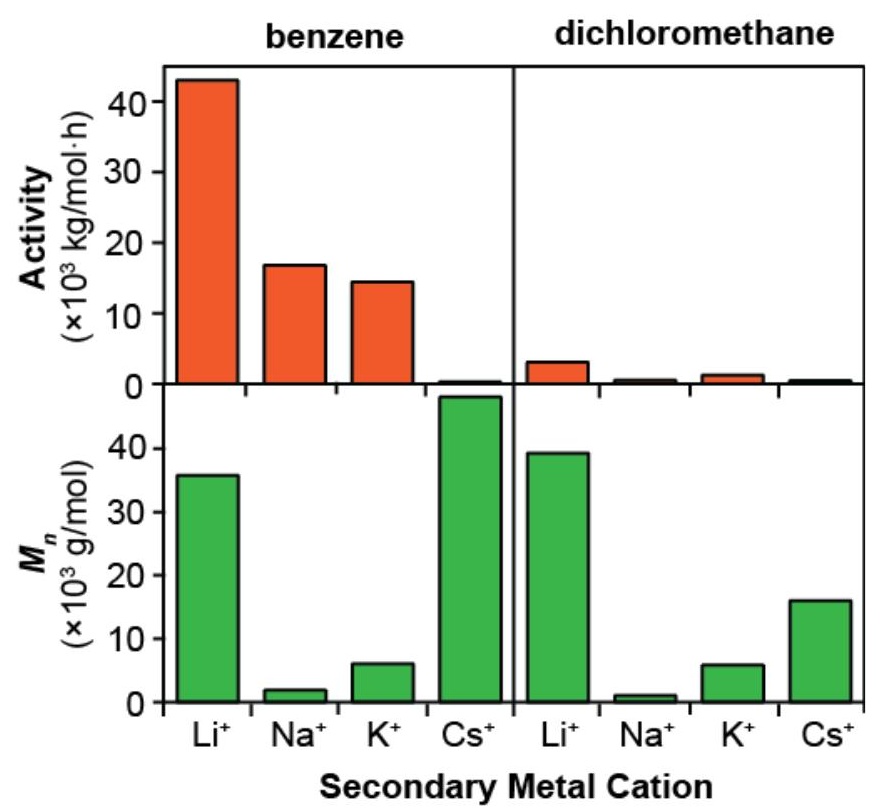

Figure S7. Effect of solvent on ethylene polymerization activity catalyzed by $\mathbf{N i 3}$ and its polymer molecular weight. Full data shown in Table S5. 
Table S6. Ethylene Polymerization by Ni3-Li at Various Temperatures ${ }^{a}$

\begin{tabular}{cccccc}
\hline Entry & $\begin{array}{c}\text { Temp. } \\
\left({ }^{\circ} \mathrm{C}\right)\end{array}$ & $\begin{array}{c}\text { Polymer Yield } \\
(\mathrm{g})\end{array}$ & $\begin{array}{c}\text { Activity } \\
(\mathrm{kg} / \mathrm{mol} \cdot \mathrm{h})\end{array}$ & $\begin{array}{c}\boldsymbol{M}_{\boldsymbol{n}}{ }^{b} \\
\left(\times 10^{3}\right)\end{array}$ & $\boldsymbol{M}_{\boldsymbol{w}} / \boldsymbol{M}_{\boldsymbol{n}}{ }^{b}$ \\
\hline 1 & 30 & 3.53 & 35000 & 40.1 & 1.3 \\
2 & 40 & 5.18 & 52000 & 25.8 & 1.3 \\
$3^{c}$ & 40 & 6.97 & 70000 & 25.0 & 1.4 \\
$4^{d}$ & 40 & 4.47 & 89000 & 21.7 & 1.2 \\
5 & 50 & 3.84 & 38000 & 12.1 & 1.3 \\
6 & 60 & 2.29 & 23000 & 10.5 & 1.3 \\
7 & 70 & 1.82 & 18000 & 6.2 & 1.3 \\
8 & 80 & 1.89 & 19000 & 3.1 & 2.0 \\
9 & 90 & 1.31 & 13000 & 2.1 & 2.2
\end{tabular}

${ }^{a}$ Polymerization conditions: Ni3 $(0.1 \mu \mathrm{mol}), \mathrm{LiBAr}_{4}{ }_{4}(0.2 \mu \mathrm{mol}), \mathrm{Ni}(\mathrm{COD})_{2}(0.4 \mu \mathrm{mol})$, ethylene $(450 \mathrm{psi}), 100 \mathrm{~mL}$ toluene, $1 \mathrm{~h}$. Temperature was controlled by manual external cooling when necessary to ensure that the reaction temperature does not exceed greater than $5^{\circ} \mathrm{C}$ from the starting temperature. Reported yields are the average of 2-3 runs and standard deviations are less than $10 \%$. ${ }^{b}$ Determined by GPC in trichlorobenzene at $140{ }^{\circ} \mathrm{C} .{ }^{c} 200 \mathrm{~mL}$ of toluene solvent was used. ${ }^{d}$ Reaction was run for $0.5 \mathrm{~h}$.

A) Comparison of Activity and $M_{\mathrm{n}}$

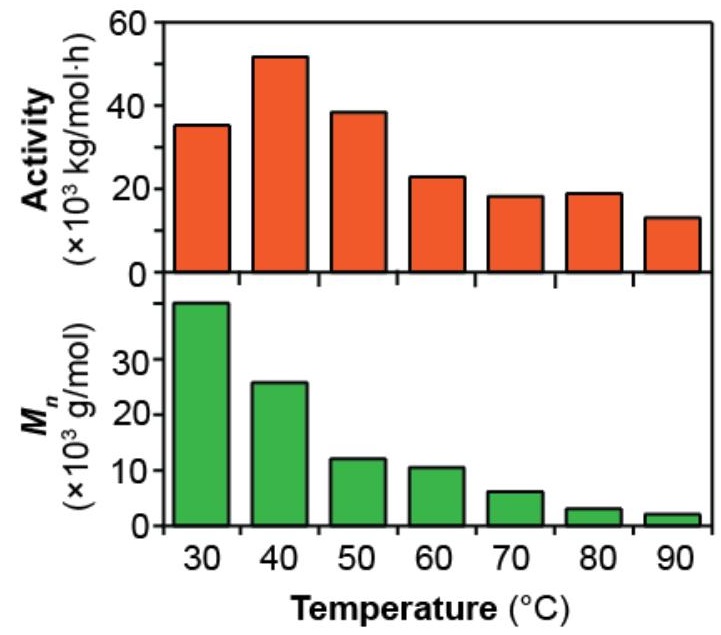

B) Optimization Study at $40^{\circ} \mathrm{C}$

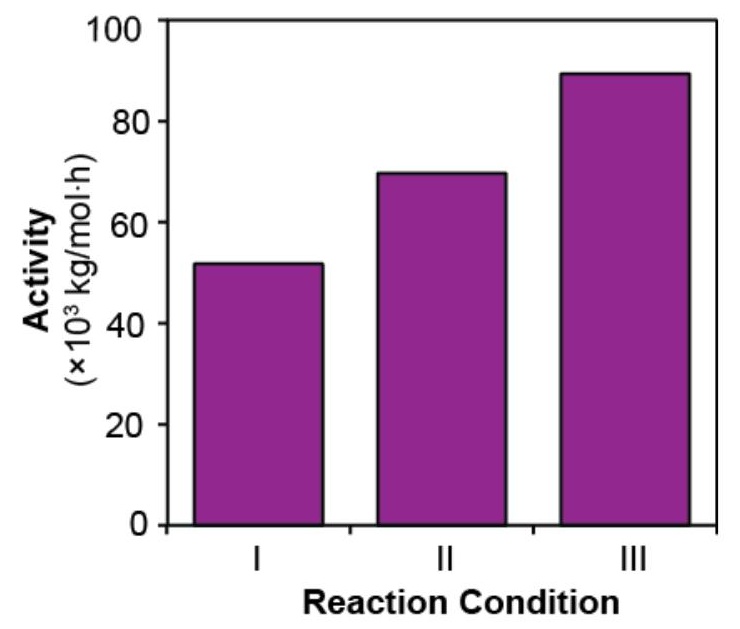

Figure S8. A) Comparison of Ni3-Li catalyst activity and its polymer $M_{\mathrm{n}}$ as a function of temperature. B) Optimization of ethylene polymerization by Ni3-Li at $40{ }^{\circ} \mathrm{C}$. Reaction Condition $\mathrm{I}=1 \mu \mathrm{M}$ catalyst, $1 \mathrm{~h}$; Reaction Condition II $=0.5 \mu \mathrm{M}$ catalyst, $2 \mathrm{~h}$; Reaction Condition III $=1$ $\mu \mathrm{M}$ catalyst, $0.5 \mathrm{~h}$. 
Table S7. Ethylene Polymerization by Ni3-Li in Various Solvents ${ }^{a}$

\begin{tabular}{ccccccc}
\hline Entry & Solvent & $\begin{array}{c}\text { Polymer } \\
\text { Yield }(\mathrm{g})\end{array}$ & $\begin{array}{c}\text { Activity } \\
(\mathrm{kg} / \mathrm{mol} \cdot \mathrm{h})\end{array}$ & $\begin{array}{c}\text { Branches }^{b} \\
(/ 1000 \mathrm{C})\end{array}$ & $\begin{array}{c}\boldsymbol{M}_{\boldsymbol{n}}{ }^{\mathrm{c}} \\
\left(\times 10^{3}\right)\end{array}$ & $\boldsymbol{M}_{\boldsymbol{w}} / \boldsymbol{M}_{\boldsymbol{n}}{ }^{c}$ \\
\hline 1 & pentane & 4.5 & 45000 & 4 & 44.02 & 1.4 \\
2 & hexane & 5.5 & 55000 & 4 & 68.15 & 1.4 \\
3 & benzene & 5.3 & 53000 & 6 & 35.76 & 1.3 \\
4 & toluene & 3.53 & 35000 & 12 & 33.93 & 1.5 \\
$5^{d}$ & $\mathrm{CH}_{2} \mathrm{Cl}{ }_{2}$ & 1.53 & 3100 & 7 & 39.30 & 1.3 \\
$6^{e}$ & $\mathrm{Et}_{2} \mathrm{O}$ & 10.8 & 11000 & 6 & 37.86 & 1.6 \\
$7^{e}$ & $\mathrm{CH}_{3} \mathrm{CN}$ & trace & 0 & - & - & -
\end{tabular}

${ }^{a}$ Polymerization conditions: Ni3 $(0.1 \mu \mathrm{mol}), \mathrm{LiBAr}_{4}{ }_{4}(0.2 \mu \mathrm{mol}), \mathrm{Ni}(\mathrm{COD})_{2}(0.8 \mu \mathrm{mol})$, ethylene $(450 \mathrm{psi}), 100 \mathrm{~mL}$ solvent, $30{ }^{\circ} \mathrm{C}, 1 \mathrm{~h}$. Temperature was controlled by manual external cooling when necessary to ensure that the reaction temperature does not exceed greater than $5^{\circ} \mathrm{C}$ from the starting temperature. Reported yields are the average of 2-3 runs and standard deviations are less than $10 \%$. ${ }^{b}$ The total number of branches per 1000 carbons was determined by ${ }^{1} \mathrm{H}$ NMR spectroscopy. ${ }^{c}$ Determined by GPC in trichlorobenzene at $140{ }^{\circ} \mathrm{C}$. ${ }^{d} \mathrm{Complex}$ Ni3 $(0.5$ $\mu \mathrm{mol}), \mathrm{LiBAr}_{4}(1 \mu \mathrm{mol}), \mathrm{Ni}(\mathrm{COD})_{2}(4 \mu \mathrm{mol}) .{ }^{e} \mathrm{Complex} \mathrm{Ni3}(1 \mu \mathrm{mol}), \operatorname{LiBAr}_{4}(2 \mu \mathrm{mol}), \mathrm{Ni}(\mathrm{COD})_{2}(8 \mu \mathrm{mol})$. 
Table S8. Ethylene Polymerization Catalyzed by Ni3 and Various Equivalents of Salts ${ }^{a}$

\begin{tabular}{ccccccc}
\hline Entry & $\begin{array}{c}\text { Ni3 } \\
(\mu \mathrm{mol})\end{array}$ & $\begin{array}{c}\text { Ni[COD }]_{2} \\
(\mu \mathrm{mol})\end{array}$ & Salt & $\begin{array}{c}\text { Salt Amount } \\
(\text { equiv. })\end{array}$ & $\begin{array}{c}\text { Polymer } \\
\text { Yield }(\mathrm{g})\end{array}$ & $\begin{array}{c}\text { Activity } \\
(\mathrm{kg} / \mathrm{mol} \cdot \mathrm{h})\end{array}$ \\
\hline 1 & 0.5 & 4 & $\mathrm{LiBAr}_{4}$ & 2 & 2.03 & 12000 \\
2 & 0.5 & 4 & $\mathrm{LiBAr}_{4}$ & 10 & 3.38 & 20000 \\
3 & 0.5 & 4 & $\mathrm{LiBAr}_{4}$ & 20 & 3.54 & 21000 \\
4 & 5 & 20 & $\mathrm{NaBAr}_{4}$ & 2 & 2.10 & 1300 \\
5 & 5 & 20 & $\mathrm{NaBAr}_{4}$ & 10 & 4.53 & 2700 \\
6 & 5 & 20 & $\mathrm{NaBAr}_{4}$ & 20 & 6.60 & 4000 \\
7 & 5 & 20 & $\mathrm{KBAr}_{4}$ & 2 & 0.49 & 290 \\
8 & 5 & 20 & $\mathrm{KBAr}_{4}$ & 10 & 2.62 & 1600 \\
9 & 5 & 20 & $\mathrm{KBAr}_{4}$ & 20 & 3.05 & 1800
\end{tabular}

${ }^{a}$ Polymerization conditions: ethylene $(450 \mathrm{psi}), 50 \mathrm{~mL}$ toluene $/ 50 \mathrm{~mL} \mathrm{Et} 2 \mathrm{O}, 20 \mathrm{~min}, 30{ }^{\circ} \mathrm{C}$. Temperature was controlled by manual external cooling when necessary to ensure that the reaction temperature does not exceed greater than $5{ }^{\circ} \mathrm{C}$ from the starting temperature. Reported yields are the average of 2-3 runs and standard deviations are less than $10 \%$.

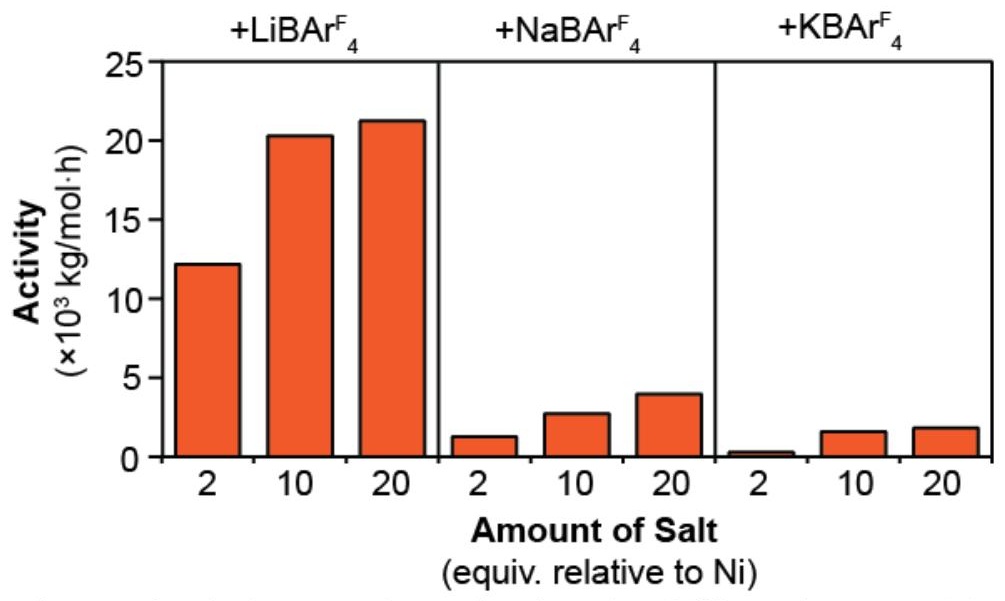

Figure S9. Comparison of ethylene polymerization by $\mathbf{N i 3}$ and up to 20 equiv. of $\mathrm{MBAr}_{4}$ relative to Ni. Full data shown in Table S8. 
Table S9. Ethylene Polymerization Catalyzed by Ni3-Li and Lithium Salts ${ }^{a}$

\begin{tabular}{|c|c|c|c|c|c|c|c|}
\hline Entry & $\begin{array}{c}\text { Salt } \\
\text { (2 equiv.) }\end{array}$ & Solvent & $\begin{array}{l}\text { Polymer } \\
\text { Yield (g) }\end{array}$ & $\begin{array}{c}\text { Activity } \\
(\mathrm{kg} / \mathrm{mol} \cdot \mathrm{h})\end{array}$ & $\begin{array}{c}\boldsymbol{M n}^{c} \\
\left(\times 10^{3}\right)\end{array}$ & $\boldsymbol{M}_{w} / \boldsymbol{M}^{c}$ & $\begin{array}{c}\text { Branches }^{b} \\
(/ 1000 \mathrm{C})\end{array}$ \\
\hline 1 & $\mathrm{LiBAr}_{4}{ }_{4}$ & toluene & 12.08 & 24000 & 13.3 & 1.7 & - \\
\hline 2 & $\mathrm{LiB}\left(\mathrm{C}_{6} \mathrm{~F}_{5}\right)_{4}$ & toluene & 10.05 & 20000 & 11.6 & 1.4 & - \\
\hline 3 & $\mathrm{LiBPh}_{4}$ & toluene & 5.36 & 11000 & 15.6 & 1.3 & - \\
\hline 4 & LiOTf & toluene & trace & 0 & - & - & - \\
\hline $5^{c}$ & $\mathrm{LiBAr}_{4}$ & $\begin{array}{c}\text { toluene/ } / \mathrm{Et}_{2} \mathrm{O} \\
(1: 1)\end{array}$ & 8.96 & 18000 & 27.2 & 1.4 & 10 \\
\hline $6^{c}$ & LiTPFB & $\begin{array}{c}\text { toluene/ } \mathrm{Et}_{2} \mathrm{O} \\
(1: 1)\end{array}$ & 8.01 & 16000 & 29.2 & 1.4 & 8 \\
\hline $7^{c}$ & $\mathrm{LiBPh}_{4}$ & $\begin{array}{c}\text { toluene/ } / \mathrm{Et}_{2} \mathrm{O} \\
(1: 1)\end{array}$ & 3.89 & 7800 & 32.2 & 1.3 & 4 \\
\hline $8^{c}$ & LiOTf & $\begin{array}{c}\text { toluene/ } \mathrm{Et}_{2} \mathrm{O} \\
(1: 1)\end{array}$ & trace & 0 & - & - & - \\
\hline
\end{tabular}

${ }^{a}$ Polymerization conditions: Ni3 $(0.5 \mu \mathrm{mol}), \mathrm{Ni}[\mathrm{COD}]_{2}(4 \mu \mathrm{mol})$, ethylene $(450 \mathrm{psi}), 100 \mathrm{~mL}$ of solvent, $1 \mathrm{~h}$, start reaction at $30{ }^{\circ} \mathrm{C}$. Temperature was not controlled by manual external cooling. Reported yields are the average of 2 3 runs and standard deviations are less than $10 \% .{ }^{b}$ The total number of branches per 1000 carbons was determined by ${ }^{1} \mathrm{H}$ NMR spectroscopy. ${ }^{c}$ Temperature was controlled by manual external cooling when necessary to ensure that the reaction temperature does not exceed greater than $10^{\circ} \mathrm{C}$ from the starting temperature.

Table S10. Time Study of Ethylene Polymerization Catalyzed by Ni3-Li ${ }^{a}$

\begin{tabular}{cccc}
\hline Entry & $\begin{array}{c}\text { Time } \\
(\mathrm{min})\end{array}$ & $\begin{array}{c}\text { Polymer } \\
\text { Yield }(\mathrm{g})\end{array}$ & $\begin{array}{c}\text { Activity } \\
(\mathrm{kg} / \mathrm{mol} \cdot \mathrm{h})\end{array}$ \\
\hline 1 & 8 & 2.80 & 42000 \\
2 & 13 & 7.26 & 67000 \\
3 & 14 & 8.93 & 76000 \\
4 & 19 & 9.59 & 60000 \\
5 & 27 & 13.74 & 61000 \\
6 & 60 & 12.08 & 24000
\end{tabular}

${ }^{a}$ Polymerization conditions: Ni3 $(0.5 \mu \mathrm{mol}), \mathrm{LiBAr}_{4}(1.0 \mu \mathrm{mol}), \mathrm{Ni}[\mathrm{COD}]_{2}(4 \mu \mathrm{mol})$, ethylene $(450 \mathrm{psi}), 100 \mathrm{~mL}$ toluene, $1 \mathrm{~h}$, start reaction at $30^{\circ} \mathrm{C}$. Temperature was not controlled by manual external cooling. 
Table S11. Comparison of Highly Active Nickel Ethylene Polymerization Catalysts Reported in the Literature
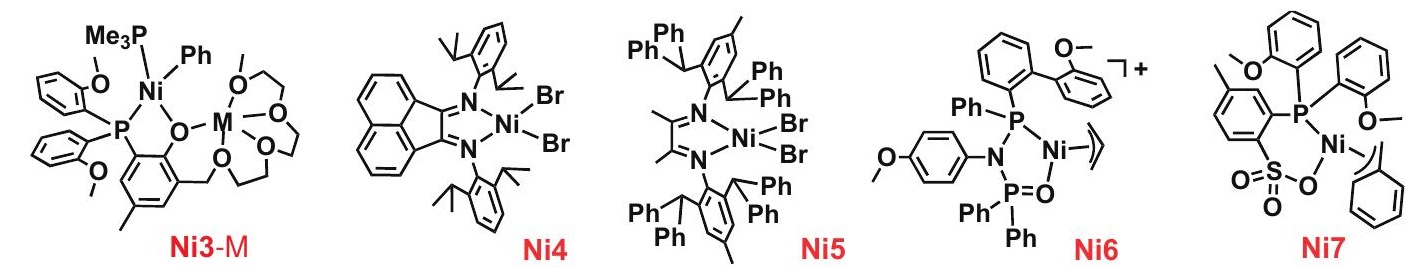

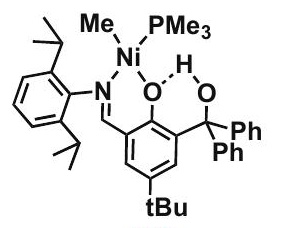

Ni8

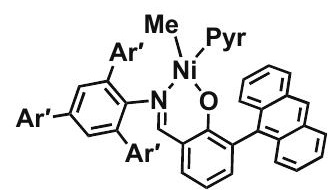

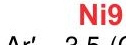

$\mathrm{Ar}^{\prime}=3,5-\left(\mathrm{CF}_{3}\right) \mathrm{C}_{6}$

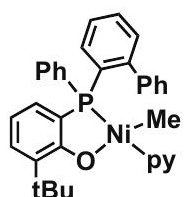

Ni10
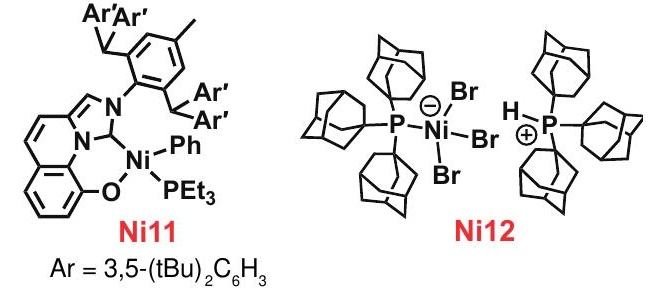

$\mathrm{Ni} 12$

$\mathrm{Ar}=3,5-(\mathrm{tBu})_{2} \mathrm{C}_{6} \mathrm{H}_{3}$

\begin{tabular}{|c|c|c|c|c|c|c|c|c|}
\hline $\begin{array}{c}\text { Complex } \\
\text { (conc.) }\end{array}$ & $\begin{array}{c}\mathrm{C}_{2} \mathbf{H}_{4} \\
\text { Pressure } \\
\quad(\mathrm{psi})\end{array}$ & $\begin{array}{c}\text { Temp. } \\
\left({ }^{\circ} \mathrm{C}\right)\end{array}$ & $\begin{array}{l}\text { Time } \\
(\mathrm{min})\end{array}$ & $\begin{array}{c}\text { Activity } \\
(\mathrm{kg} \mathrm{PE} / \mathrm{mol} \\
\mathrm{Ni} \cdot \mathrm{h})\end{array}$ & $\begin{array}{c}\text { TON } \\
\left(\times 10^{3} \mathrm{~mol}\right. \\
\text { ethylene/ } \\
\text { mol Ni })\end{array}$ & $M_{n}$ & $M_{w} / M_{n}$ & $\begin{array}{c}\text { Reference } \\
\text { (compound number in } \\
\text { original reference) }\end{array}$ \\
\hline Ni3-Na $(0.50 \mu \mathrm{mol}$ in $100 \mathrm{~mL})$ & 450 & 30 & 60 & 18100 & 646 & 1710 & 1.5 & $3($ Ni11-Na) \\
\hline Ni3-Li $(0.10 \mu \mathrm{mol}$ in $200 \mathrm{~mL})$ & 450 & 40 & 60 & 69700 & 2484 & 25000 & 1.4 & This work \\
\hline Ni3-Cs $(0.50 \mu \mathrm{mol}$ in $100 \mathrm{~mL})$ & 450 & 70 & 30 & 36480 & 650 & 15120 & 1.7 & This work \\
\hline $\mathbf{N i 4}(0.83 \mu \mathrm{mol}$ in $200 \mathrm{~mL})$ & 200 & 35 & 10 & 67200 & 400 & 337000 & 1.8 & $4(\mathbf{4 g})$ \\
\hline $\operatorname{Ni5}(1.57 \mu \mathrm{mol}$ in $100 \mathrm{~mL})$ & 100 & 100 & 10 & 2856 & 17 & 422000 & 1.2 & $5(2 b)$ \\
\hline Ni6 $(5.00 \mu \mathrm{mol}$ in $20 \mathrm{~mL})$ & 118 & 25 & 60 & 260 & 9 & 188900 & 2.5 & $6(\mathbf{N i 4})$ \\
\hline Ni7 $(20.0 \mu \mathrm{mol}$ in $30 \mathrm{~mL})$ & 300 & 25 & 120 & 163 & 12 & 1500 & - & $7(4 a)$ \\
\hline $\operatorname{Ni8}(10.0 \mu \mathrm{mol}$ in $25 \mathrm{~mL})$ & 118 & 25 & 40 & 1184 & 28 & 6700 & 1.8 & $8(\mathbf{1 b})$ \\
\hline Ni9 $(5.00 \mu \mathrm{mol}$ in $100 \mathrm{~mL})$ & 580 & 30 & 40 & 1218 & 29 & 466100 & 1.6 & $9\left(\mathbf{2}-\mathbf{C F}_{\mathbf{3}} / \mathbf{P y}\right)$ \\
\hline $\operatorname{Ni10}(5.00 \mu \mathrm{mol}$ in $100 \mathrm{~mL})$ & 145 & 30 & 20 & 2100 & 25 & 398000 & 1.5 & $10(\mathbf{2 c})$ \\
\hline $\operatorname{Ni11}(2.50 \mu \mathrm{mol}$ in $8.5 \mathrm{~mL})$ & 580 & 30 & 30 & 1000 & 18 & 84000 & 2.0 & $11(7 \mathrm{c})$ \\
\hline $\operatorname{Ni12}(0.50 \mu \mathrm{mol}$ in $150 \mathrm{~mL})$ & 400 & 10 & 3.5 & 103600 & 216 & 1390000 & 1.4 & $12(6)$ \\
\hline
\end{tabular}


Table S12. Comparison of Thermally Stable Nickel Ethylene Polymerization Catalysts Reported in the Literature
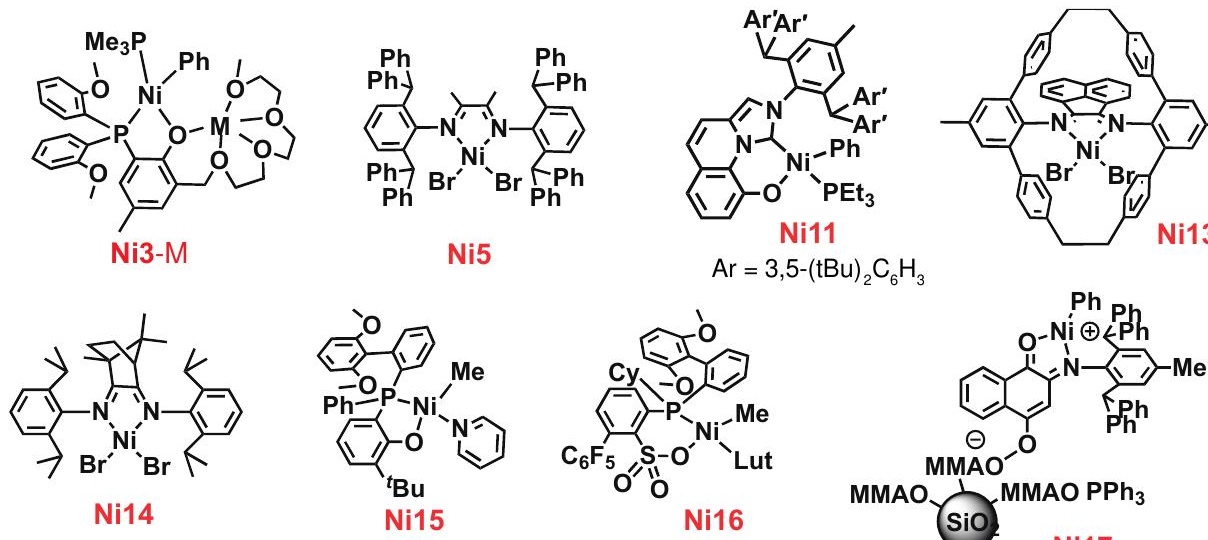

Ni14

$\mathrm{Ni} 15$

$\mathrm{Ni} 16$
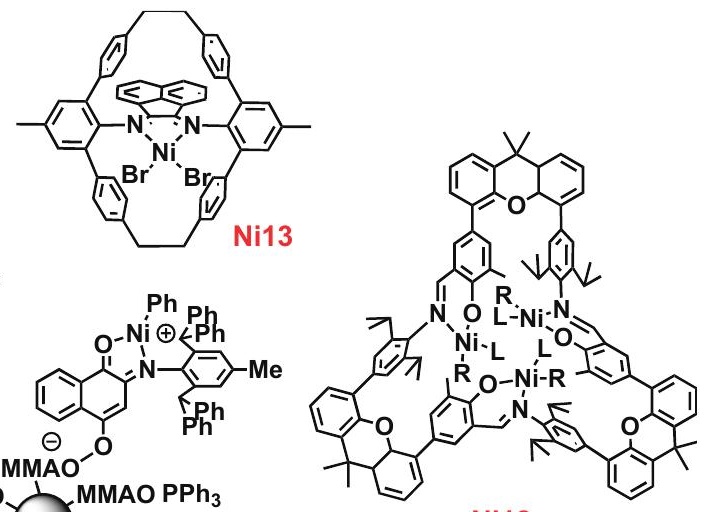

Ni18

\begin{tabular}{|c|c|c|c|c|c|c|c|c|}
\hline $\begin{array}{c}\text { Complex } \\
\text { (conc.) }\end{array}$ & $\begin{array}{c}\mathrm{C}_{2} \mathrm{H}_{4} \\
\text { Pressure } \\
\text { (psi) }\end{array}$ & $\begin{array}{c}\text { Temp. } \\
\left({ }^{\circ} \mathrm{C}\right)\end{array}$ & $\begin{array}{l}\text { Time } \\
(\min )\end{array}$ & $\begin{array}{l}\text { Activity } \\
(\mathrm{kg} \mathrm{PE} / \mathrm{mol} \\
\mathrm{Ni} \cdot \mathrm{h})\end{array}$ & $\begin{array}{c}\text { TON } \\
\left(\times 10^{3} \mathrm{~mol}\right. \\
\text { ethylene/ } \\
\text { mol Ni })\end{array}$ & $M_{n}$ & $M_{w} / M_{n}$ & $\begin{array}{l}\text { Reference Number } \\
\text { (Compound name in } \\
\text { original reference) }\end{array}$ \\
\hline Ni3-Cs $(0.5 \mu \mathrm{mol}$ in $100 \mathrm{~mL})$ & 450 & 70 & 30 & 36480 & 650 & 15120 & 1.7 & This work \\
\hline Ni3-Cs $(0.5 \mu \mathrm{mol}$ in $100 \mathrm{~mL})$ & 450 & 90 & 30 & 22920 & 408 & 15740 & 1.6 & This work \\
\hline $\operatorname{Ni5}(1.57 \mu \mathrm{mol}$ in $100 \mathrm{~mL})$ & 100 & 100 & 10 & 2856 & 17 & 422000 & 1.2 & $5(2 \mathbf{b})$ \\
\hline $\operatorname{Ni11}(2.50 \mu \mathrm{mol}$ in $8.5 \mathrm{~mL})$ & 580 & 100 & 30 & 720 & 13 & 16000 & 2.3 & $11(\mathbf{7 c})$ \\
\hline $\operatorname{Ni13}(1.00 \mu \mathrm{mol}$ in $100 \mathrm{~mL})$ & 200 & 90 & 15 & 18513 & 165 & 292000 & 1.4 & $13(\mathbf{1})$ \\
\hline $\operatorname{Ni14}(5.00 \mu \mathrm{mol}$ in $20 \mathrm{~mL})$ & 7.3 & 80 & 30 & 2516 & 49 & 233200 & 2.7 & $14(\mathbf{4 b})$ \\
\hline $\operatorname{Ni15}(5.00 \mu \mathrm{mol}$ in $100 \mathrm{~mL})$ & 145 & 90 & 20 & 10020 & 119 & 9500 & 2.7 & $10(\mathbf{2 b})$ \\
\hline Ni16 $(2.00 \mu \mathrm{mol}$ in $50 \mathrm{~mL})$ & 118 & 100 & 30 & 4000 & 71 & 3800 & 3.5 & $15(4)$ \\
\hline $\operatorname{Ni17}(5.00 \mu \mathrm{mol}$ in $30 \mathrm{~mL})$ & 145 & 80 & 5 & 2000 & 6 & 16800 & 2.1 & $16(\mathbf{1})$ \\
\hline Ni18 $(40.0 \mu \mathrm{mol}$ in $20 \mathrm{~mL})$ & 90 & 100 & 10 & 27 & 0.2 & 9880 & 3.4 & $17\left(\mathbf{3}-\mathbf{N i}_{3}\right)$ \\
\hline
\end{tabular}




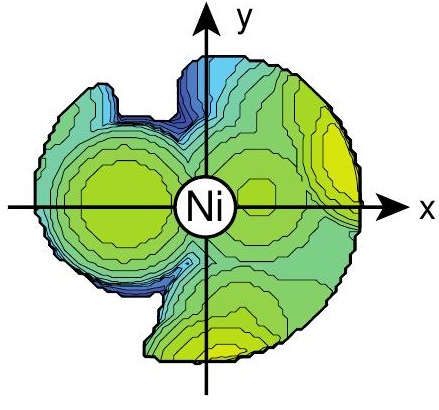

Ni1'

$\% \mathrm{~V}_{\text {bur }}=43.9$
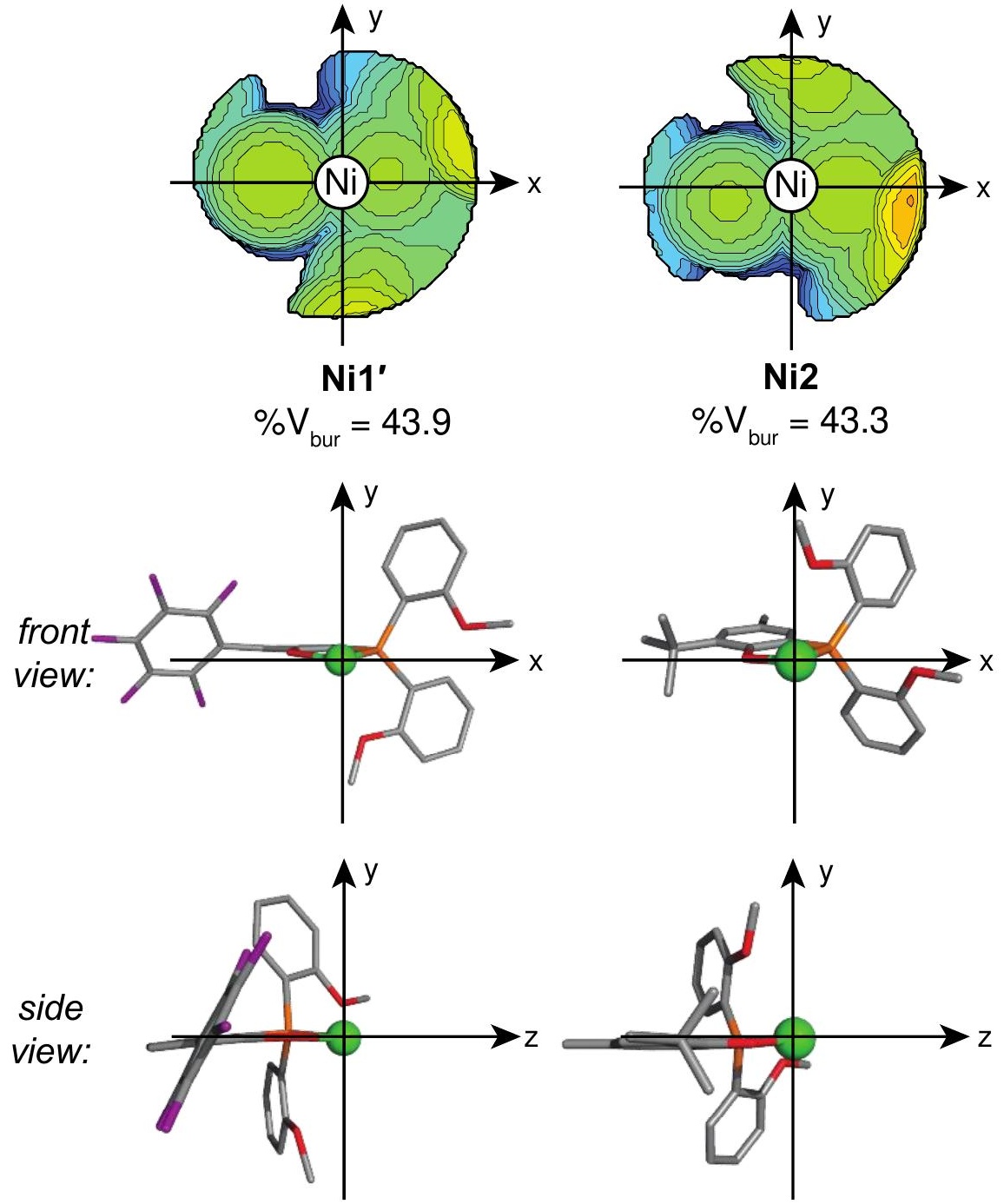

$\mathrm{Ni} 2$

$\% \mathrm{~V}_{\text {bur }}=43.3$
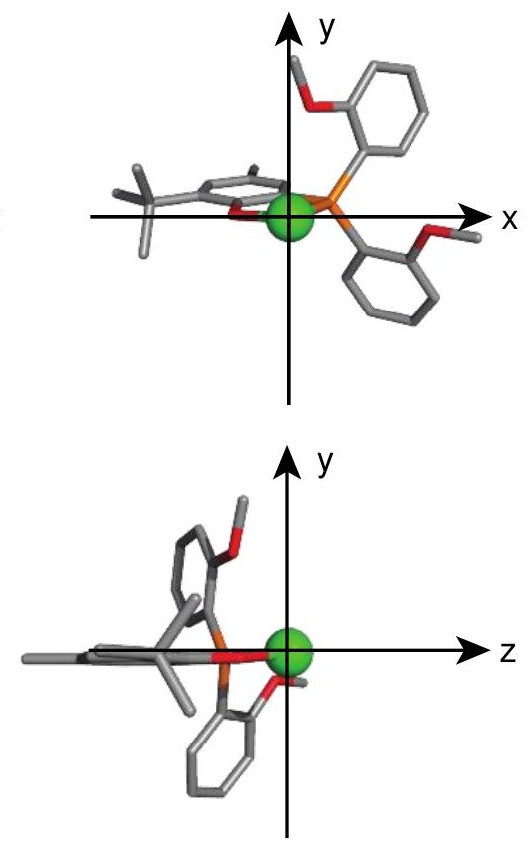

Figure S10. Topographic steric maps of Ni1' and Ni2 calculated from their x-ray structures using SambVca $2.1 .{ }^{17}$ Only the

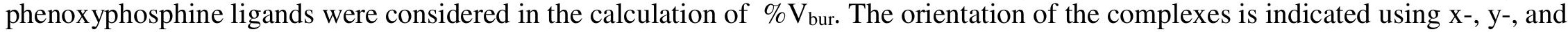
$\mathrm{z}$-coordinate axes. Atom colors: green $=$ nickel, orange $=$ phosphorous, red $=$ oxygen, purple $=$ fluorine, gray $=$ carbon. The crystallographic data for $\mathrm{Ni}^{\prime 16}$ and $\mathbf{N i 2}{ }^{15}$ were obtained from published data. 


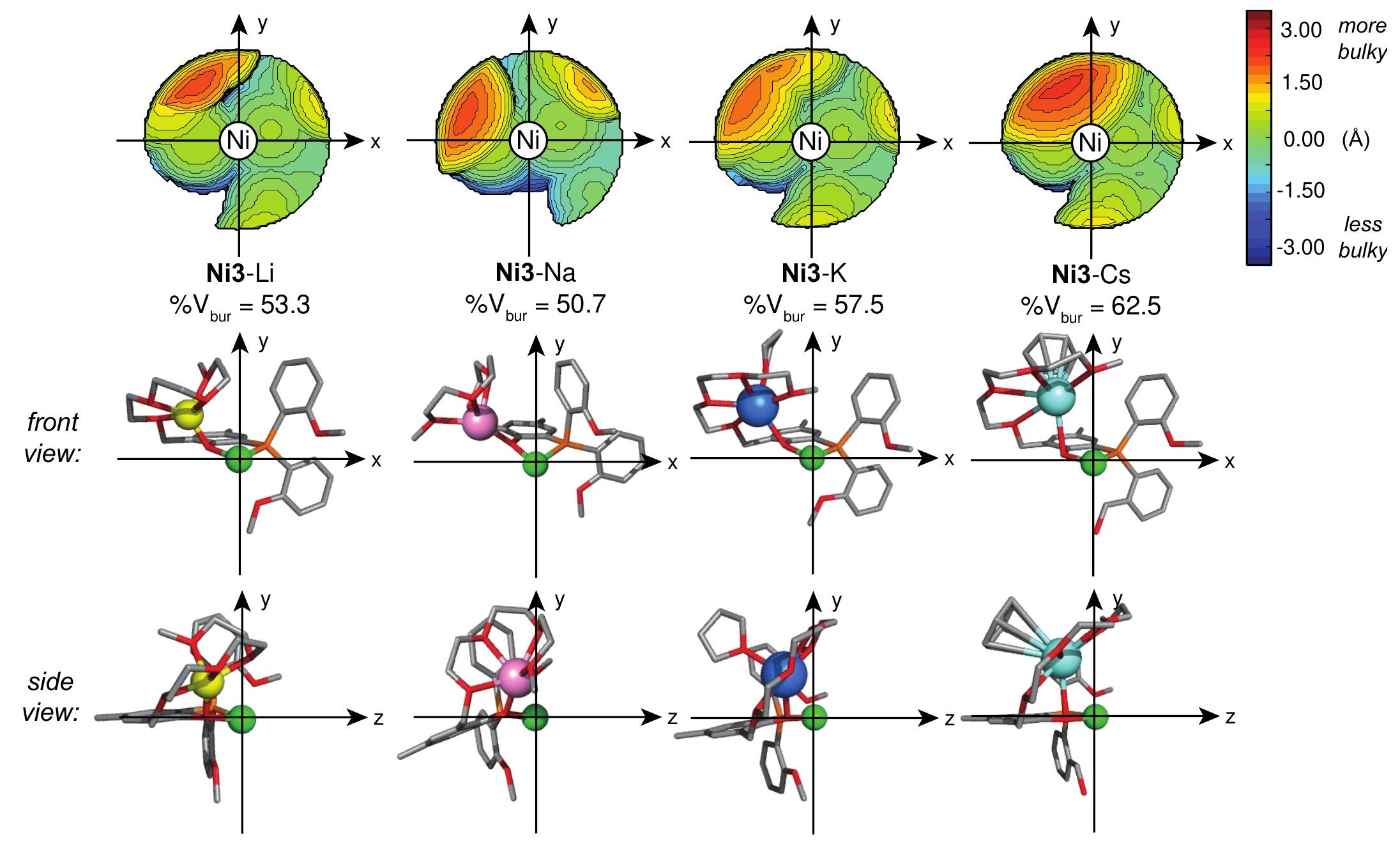

Figure S11. Topographic steric maps of Ni3-M calculated from their x-ray structures using SambVca $2.1 . .^{15}$ Only the phenoxyphosphine ligands were considered in the calculation of $\% \mathrm{~V}_{\text {bur. }}$. The orientation of the complexes is indicated using $\mathrm{x}-, \mathrm{y}-$, and $\mathrm{z}$-coordinate axes. Atom colors: green $=$ nickel, orange $=$ phosphorous, red $=$ oxygen, purple $=$ fluorine, gray $=$ carbon, yellow $=$ lithium, pink $=$ sodium, blue $=$ potassium, cyan $=$ cesium. The crystallographic data for Ni3-Na was obtained from published data ${ }^{16}$ 


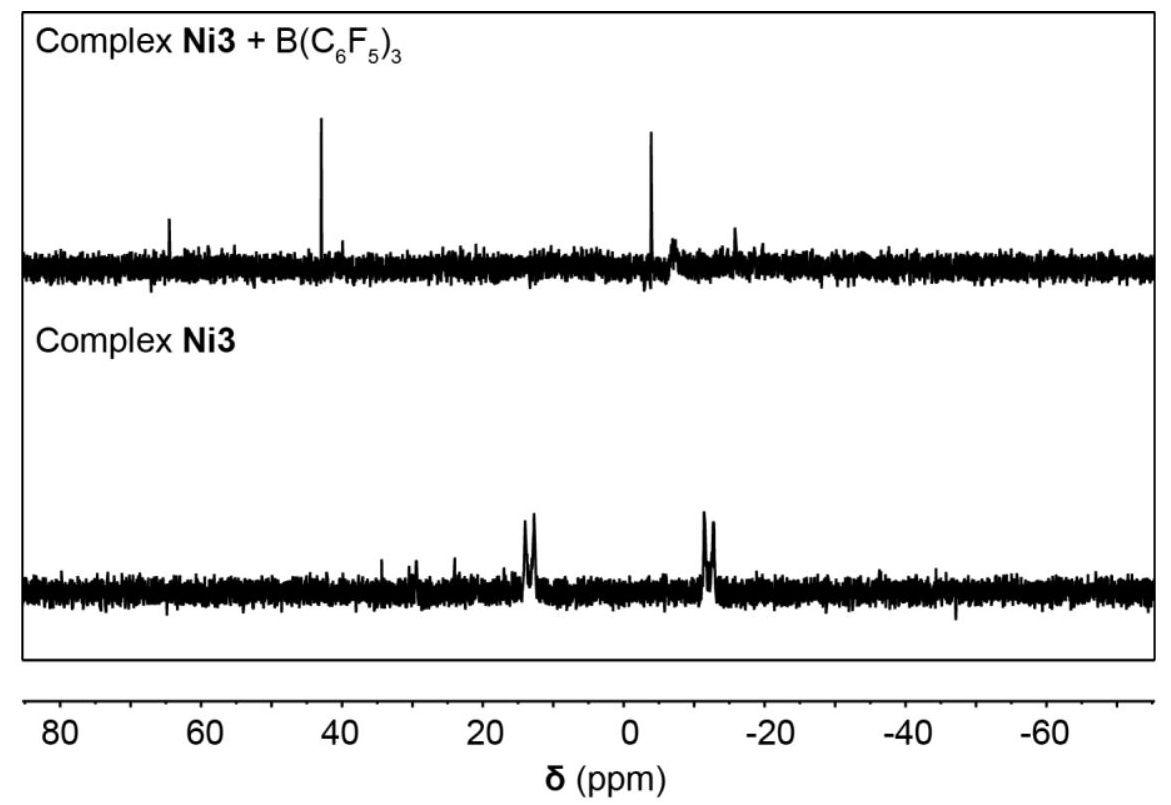

Figure S12. ${ }^{31} \mathrm{P}$ NMR spectra (toluene- $d_{8} / \mathrm{Et}_{2} \mathrm{O} 4: 1,243 \mathrm{MHz}$ ) of Ni3 before (bottom) and after (top) reaction with $\mathrm{B}\left(\mathrm{C}_{6} \mathrm{~F}_{5}\right)_{3}$ for $5 \mathrm{~min}$ in toluene- $\mathrm{d}_{8} / \mathrm{Et}_{2} \mathrm{O}$ at $30{ }^{\circ} \mathrm{C}$. 


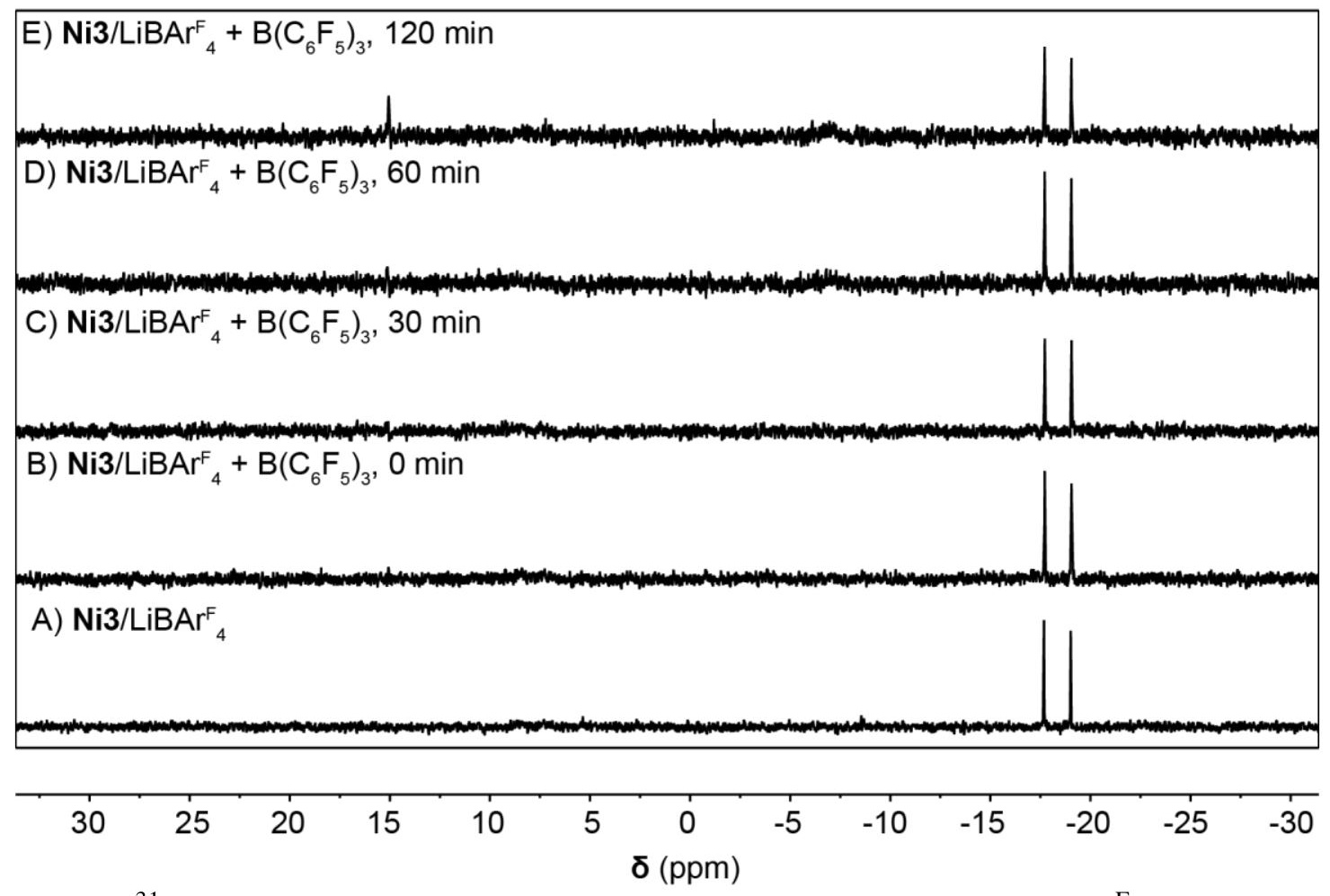

Figure S13. ${ }^{31} \mathrm{P}$ NMR spectra (toluene- $d_{8} / \mathrm{Et}_{2} \mathrm{O} 4: 1,243 \mathrm{MHz}$ ) of Ni3/LiBAr $\mathrm{F}_{4}(1: 5)$ before (A) and after $(\mathrm{B}-\mathrm{E})$ reaction with $\mathrm{B}\left(\mathrm{C}_{6} \mathrm{~F}_{5}\right)_{3}$ in toluene- $\mathrm{d}_{8} / \mathrm{Et}_{2} \mathrm{O}$ at $30{ }^{\circ} \mathrm{C}$.

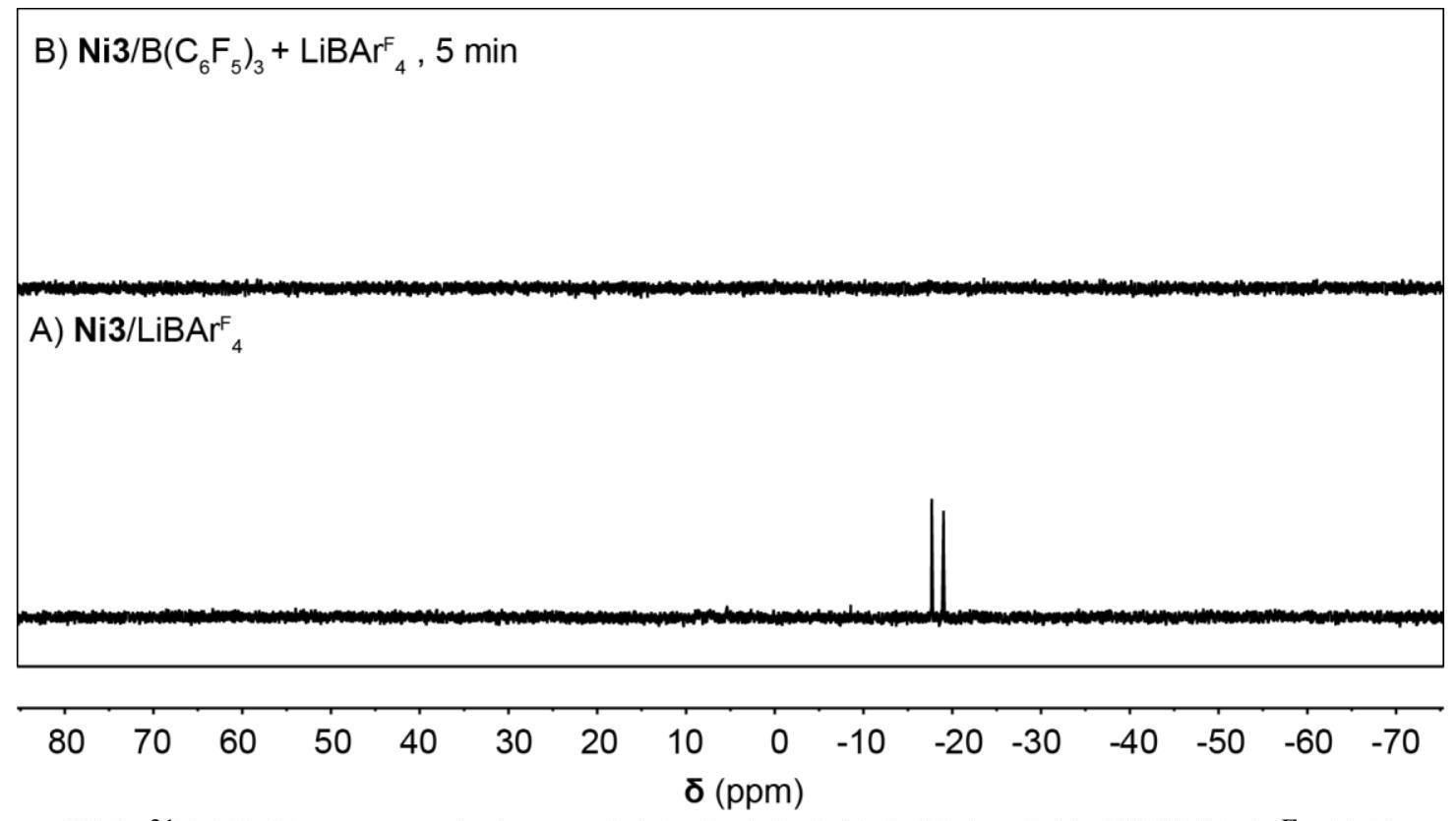

Figure S14. ${ }^{31} \mathrm{P}$ NMR spectra (toluene- $d_{8} / \mathrm{Et}_{2} \mathrm{O} 4: 1,243 \mathrm{MHz}$ ) of A) Ni3/LiBAr${ }_{4}(1: 5)$ and B) $\mathrm{Ni3} / \mathrm{B}\left(\mathrm{C}_{6} \mathrm{~F}_{5}\right)_{3}(1: 3)$ followed by the addition of $\mathrm{LiBAr}_{4}{ }_{4}$ in toluene- $\mathrm{d}_{8} / \mathrm{Et}_{2} \mathrm{O}$ at $30{ }^{\circ} \mathrm{C}$. 


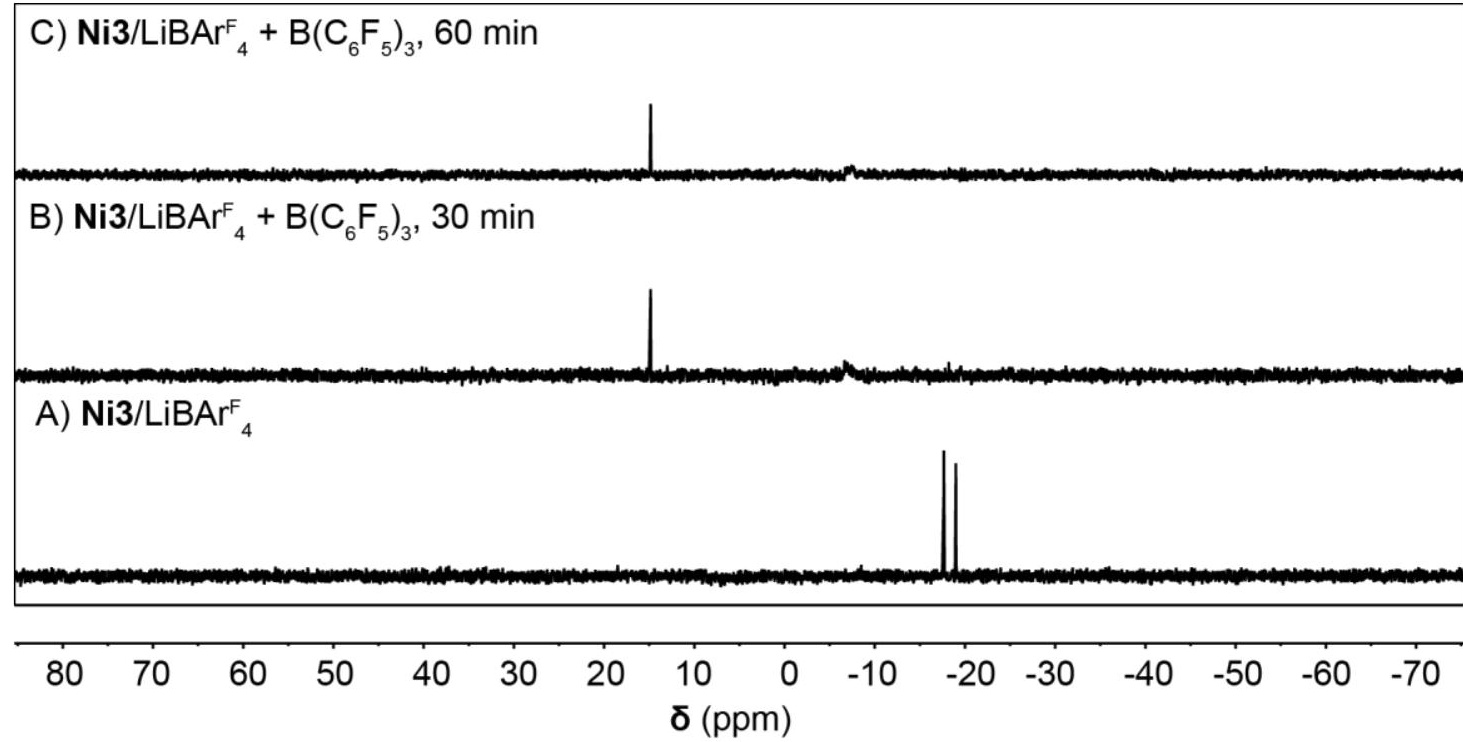

Figure S15. ${ }^{31} \mathrm{P}$ NMR spectra (toluene- $d_{8} / \mathrm{Et}_{2} \mathrm{O} 4: 1,243 \mathrm{MHz}$ ) of Ni3/LiBAr${ }_{4}$ (1:5) before (A) and after $(\mathrm{B}-\mathrm{C})$ reaction with $\mathrm{B}\left(\mathrm{C}_{6} \mathrm{~F}_{5}\right)_{3}$ in toluene- $\mathrm{d}_{8} / \mathrm{Et}_{2} \mathrm{O}$ at $50^{\circ} \mathrm{C}$.

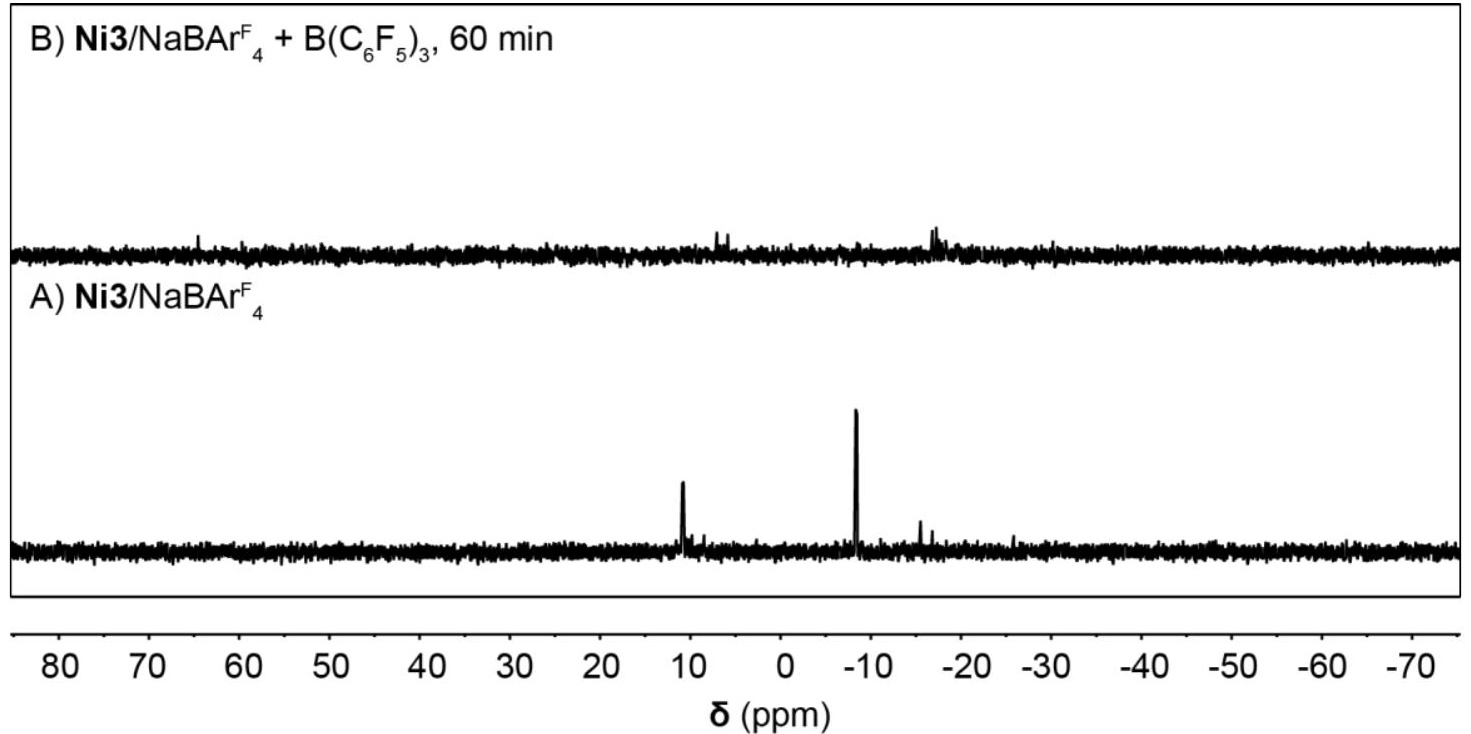

Figure S16. ${ }^{31} \mathrm{P}$ NMR spectra (toluene- $d_{8} / \mathrm{Et}_{2} \mathrm{O} 4: 1,243 \mathrm{MHz}$ ) of Ni3/NaBAr${ }_{4}$ (1:5) before (A) and after $(\mathrm{B})$ reaction with $\mathrm{B}\left(\mathrm{C}_{6} \mathrm{~F}_{5}\right)_{3}$ in toluene- $\mathrm{d}_{8} / \mathrm{Et}_{2} \mathrm{O}$ at $30{ }^{\circ} \mathrm{C}$. 


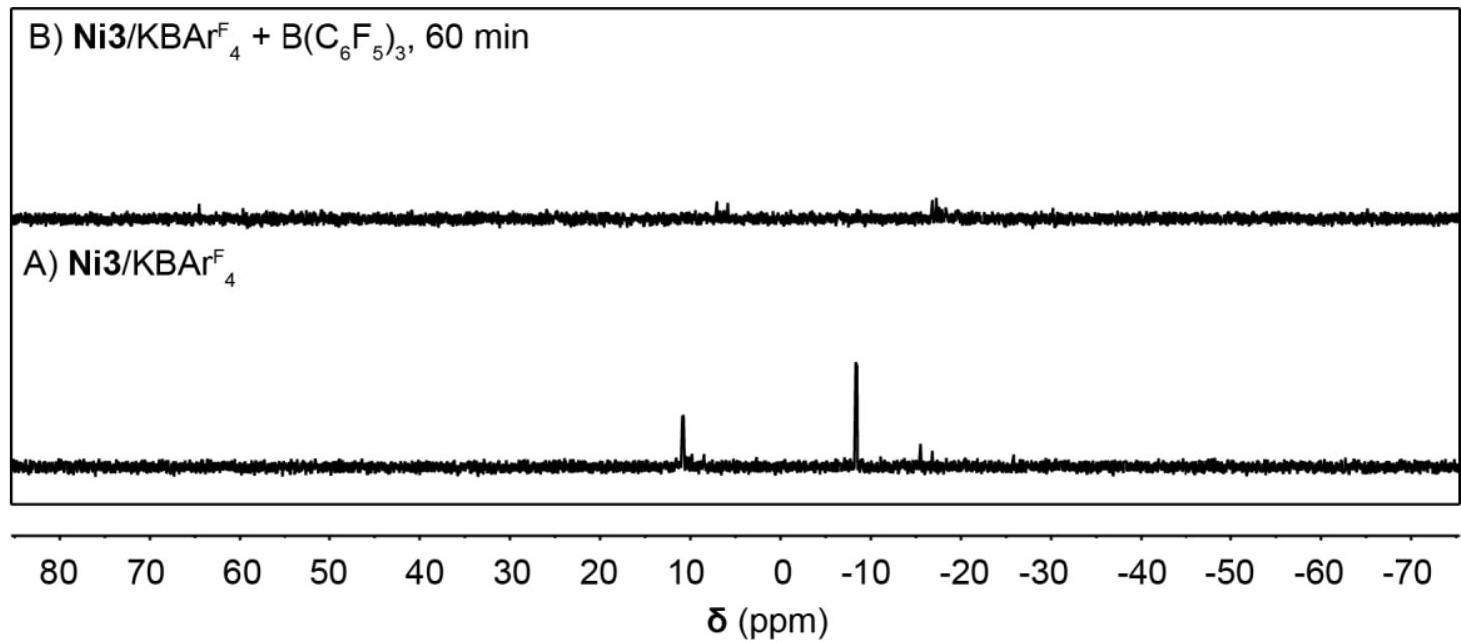

Figure S17. ${ }^{31} \mathrm{P}$ NMR spectra (toluene- $d_{8} / \mathrm{Et}_{2} \mathrm{O} 4: 1,243 \mathrm{MHz}$ ) of Ni3/KBAr${ }_{4}$ (1:5) before (A) and after $(\mathrm{B})$ reaction with $\mathrm{B}\left(\mathrm{C}_{6} \mathrm{~F}_{5}\right)_{3}$ in toluene- $\mathrm{d}_{8} / \mathrm{Et}_{2} \mathrm{O}$ at $30{ }^{\circ} \mathrm{C}$.

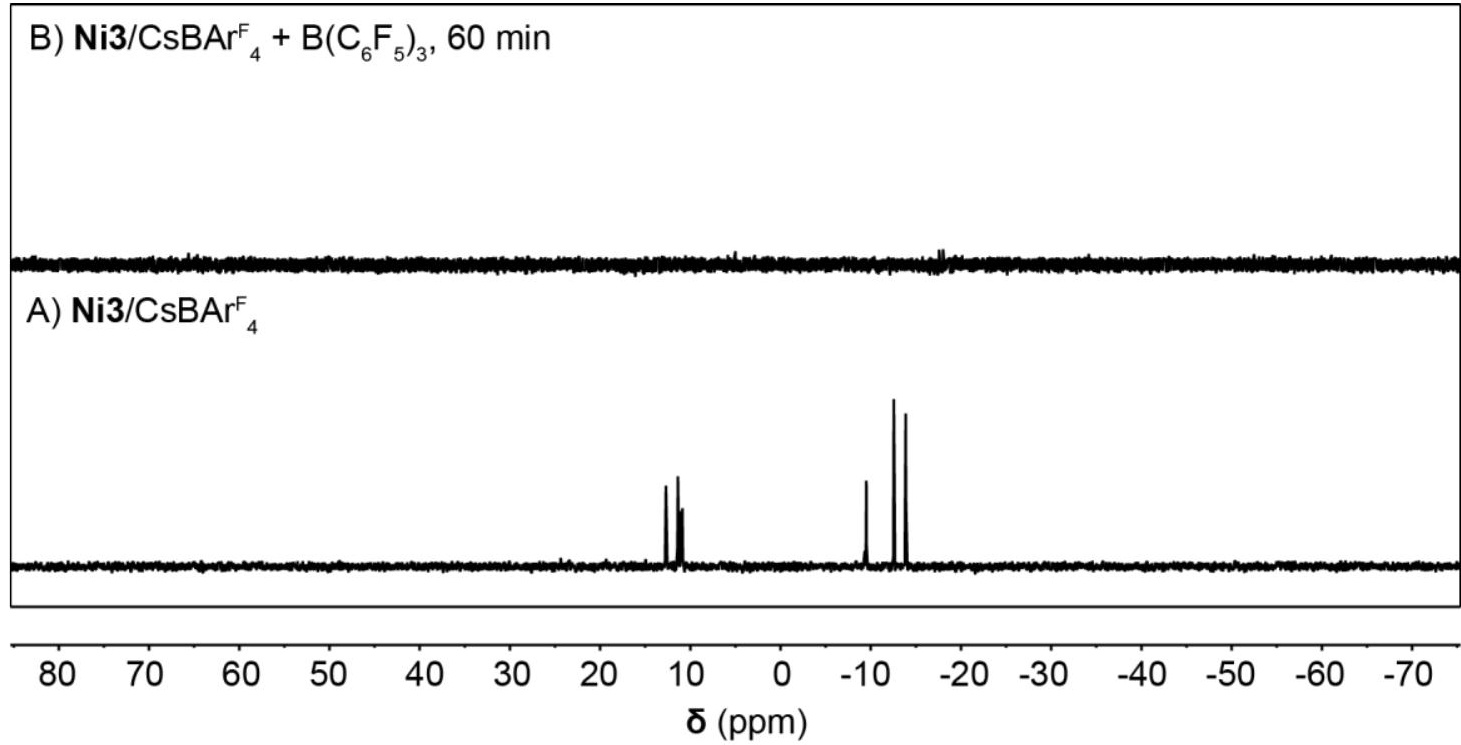

Figure S18. ${ }^{31} \mathrm{P}$ NMR spectra (toluene- $d_{8} / \mathrm{Et}_{2} \mathrm{O} 4: 1,243 \mathrm{MHz}$ ) of Ni3/CsBAr $\mathrm{F}_{4}(1: 5)$ before (A) and after $(\mathrm{B})$ reaction with $\mathrm{B}\left(\mathrm{C}_{6} \mathrm{~F}_{5}\right)_{3}$ in toluene- $\mathrm{d}_{8} / \mathrm{Et}_{2} \mathrm{O}$ at $30{ }^{\circ} \mathrm{C}$. 
Table S13. Ethylene Polymerization by Ni3-M with Different Post-Activation Times ${ }^{a}$

\begin{tabular}{|c|c|c|c|c|c|c|c|c|}
\hline \multirow{2}{*}{$\begin{array}{c}\text { Post- } \\
\text { Activation } \\
\text { Time } \\
(\text { min })\end{array}$} & \multicolumn{2}{|c|}{$\underline{\text { Ni3-Li }}$} & \multicolumn{2}{|c|}{$\underline{\mathrm{Ni3}-\mathrm{Na}}$} & \multicolumn{2}{|c|}{$\underline{\mathrm{Ni3}-\mathrm{K}}$} & \multicolumn{2}{|c|}{$\underline{\mathrm{Ni3}-\mathrm{Cs}}$} \\
\hline & $\begin{array}{c}\text { PE } \\
\text { Yield } \\
(\mathrm{g}) \\
\end{array}$ & $\begin{array}{l}\text { \% Cat. } \\
\text { Active }^{b}\end{array}$ & $\begin{array}{c}\text { PE } \\
\text { Yield } \\
(\mathrm{g}) \\
\end{array}$ & $\begin{array}{l}\% \text { Cat. } \\
\text { Active }^{b}\end{array}$ & $\begin{array}{c}\text { PE } \\
\text { Yield } \\
(\mathrm{g})\end{array}$ & $\begin{array}{l}\% \text { Cat. } \\
\text { Active }^{b}\end{array}$ & $\begin{array}{c}\text { PE } \\
\text { Yield } \\
(\mathrm{g})\end{array}$ & $\begin{array}{c}\% \\
\text { Cat. } \\
\text { Active }^{b}\end{array}$ \\
\hline 0 & 4.67 & 100 & 3.65 & 100 & 4.7 & 100 & 0.64 & 100 \\
\hline 5 & 4.5 & 96 & 2.6 & 71 & 2.53 & 54 & 0.5 & 78 \\
\hline 20 & 4.4 & 94 & 0.78 & 21 & 1.53 & 33 & 0.36 & 56 \\
\hline 60 & 0.71 & 15 & 0.19 & 5 & 0.72 & 15 & 0.19 & 30 \\
\hline
\end{tabular}

${ }^{a}$ Complex Ni3 (1 equiv.), $\mathrm{MBAr}_{4}{ }_{4}$ (5 equiv.), and $\mathrm{B}\left(\mathrm{C}_{6} \mathrm{~F}_{5}\right)_{3}$ (3 equiv.) were combined in $100 \mathrm{~mL}$ of toluene/diethyl ether (4:1) under 3-5 psi of ethylene without stirring at $50{ }^{\circ} \mathrm{C}$. After various lengths of time (post-activation time), the ethylene pressure was increased to $450 \mathrm{psi}$ and the reaction mixture was stirred for $60 \mathrm{~min} .{ }^{b}$ The $\%$ catalyst active value was calculated based on the equation: [PE yield at time $\mathrm{x}] /[\mathrm{PE}$ yield at time zero] $\times 100 \%$. 


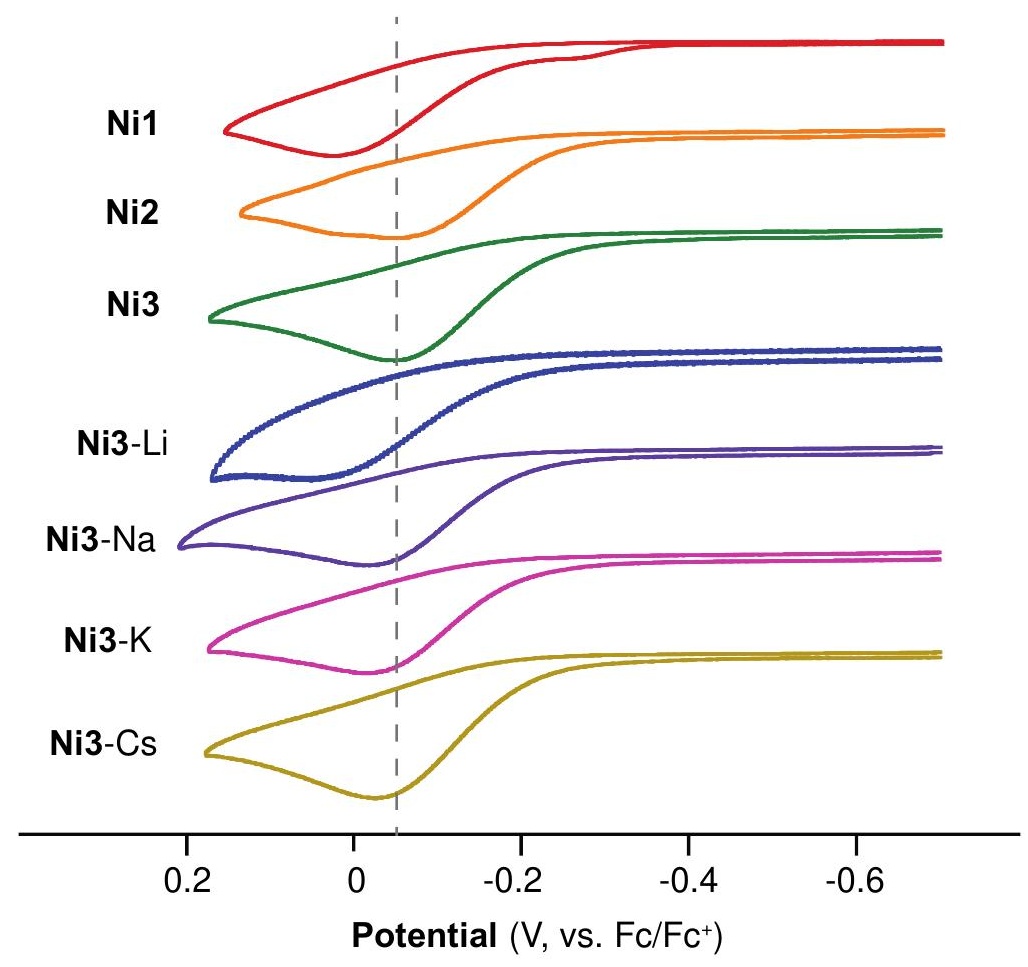

Figure S19. Cyclic voltammograms of the nickel complexes $(1 \mathrm{mM})$ recorded in THF with 0.09 $\mathrm{M} n \mathrm{Bu}_{4} \mathrm{NBPh}_{4}$ supporting electrolyte. The measurements were made using a $0.2 \mathrm{~V} / \mathrm{s}$ scan rate, a glassy carbon working electrode, a platinum wire counter electrode, and a silver wire pseudoreference. Potentials are referenced to an internal standard of ferrocene, and currents are normalized. 


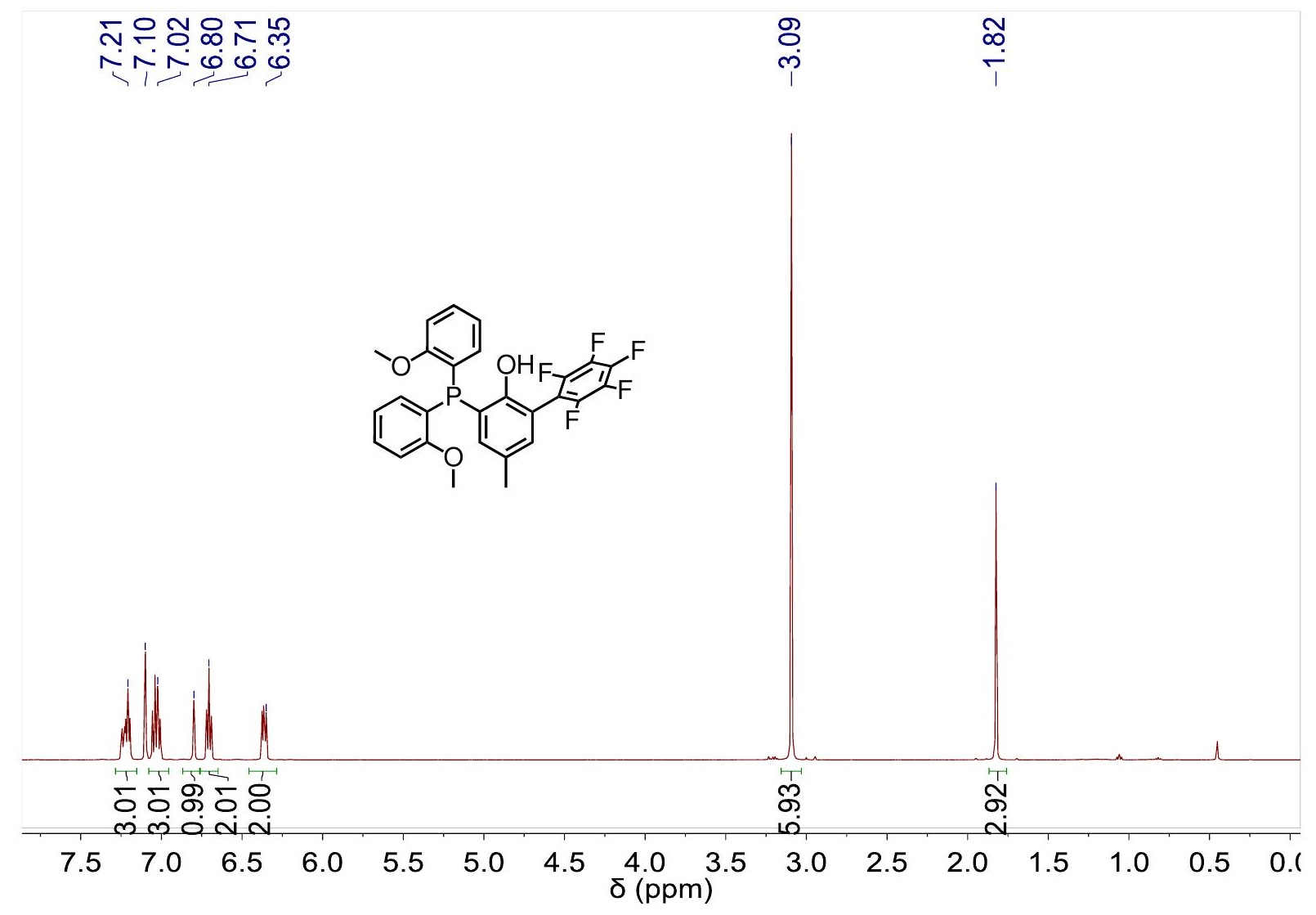

Figure S20. ${ }^{1} \mathrm{H}$ NMR spectrum $\left(\mathrm{C}_{6} \mathrm{D}_{6}, 500 \mathrm{MHz}\right)$ of compound 3. 


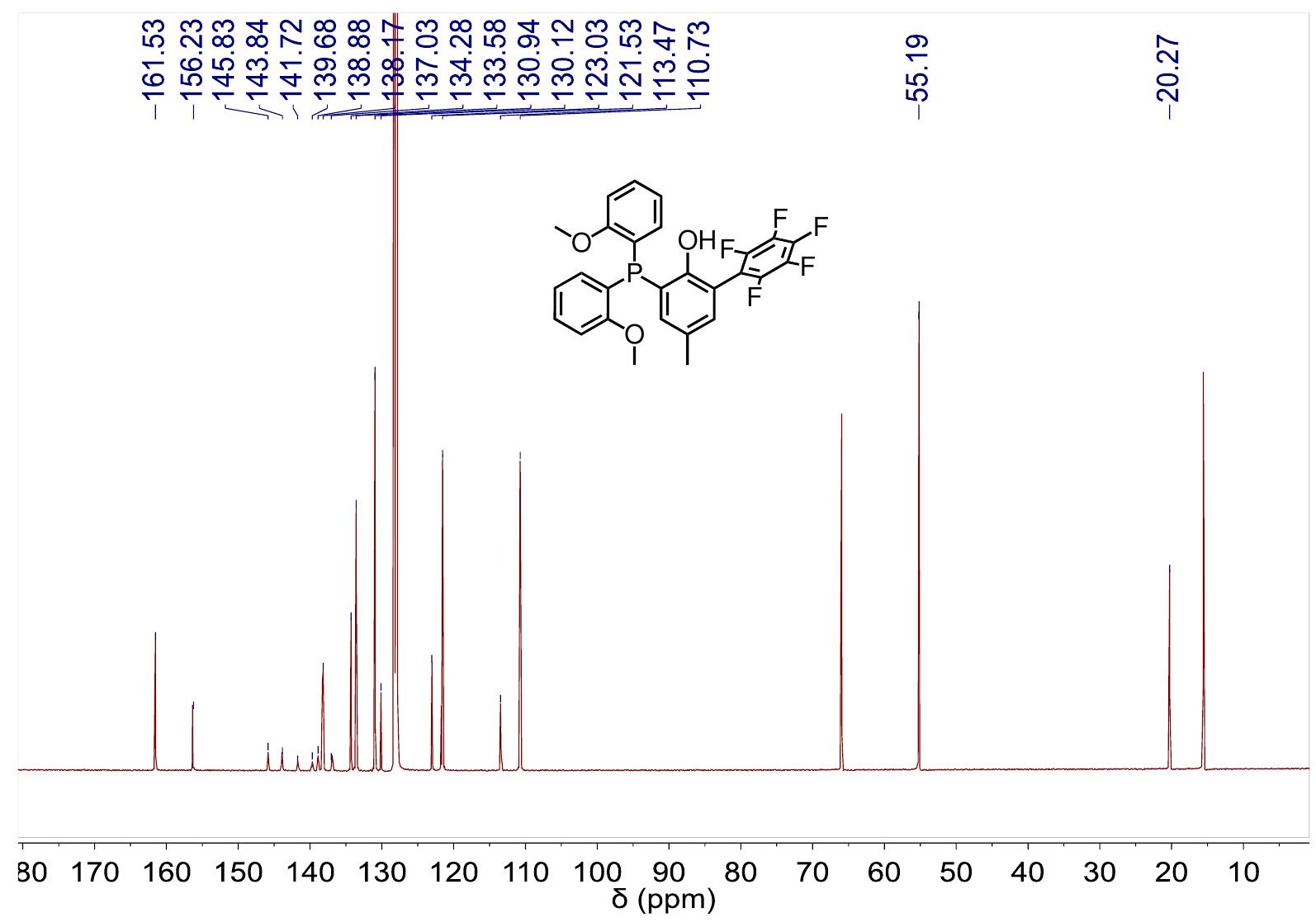

Figure S21. ${ }^{13} \mathrm{C}$ NMR spectrum $\left(\mathrm{C}_{6} \mathrm{D}_{6}, 125 \mathrm{MHz}\right)$ of compound 3. 


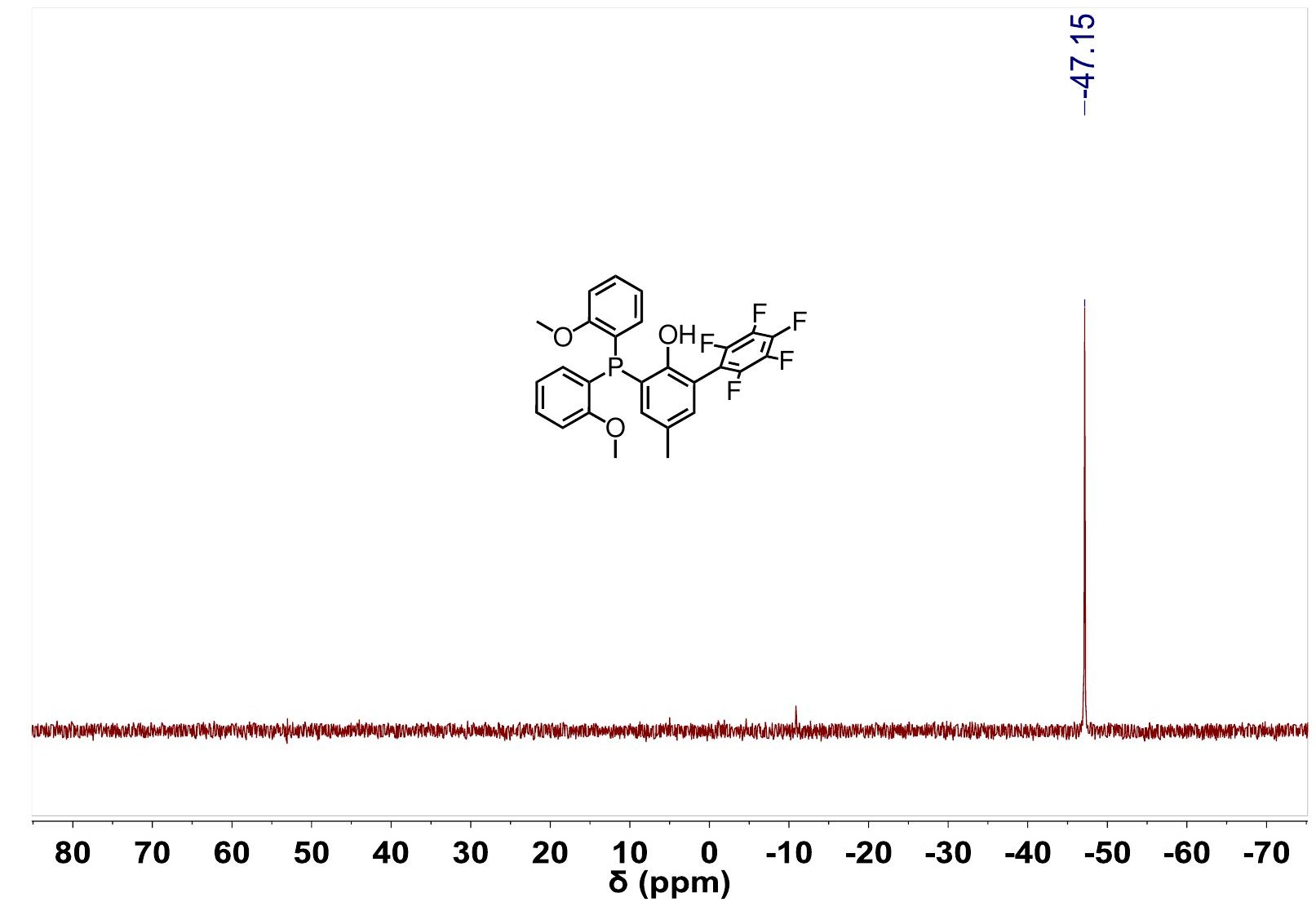

Figure S22. ${ }^{31} \mathrm{P}$ NMR spectrum $\left(\mathrm{C}_{6} \mathrm{D}_{6}, 202 \mathrm{MHz}\right)$ of compound 3 . 


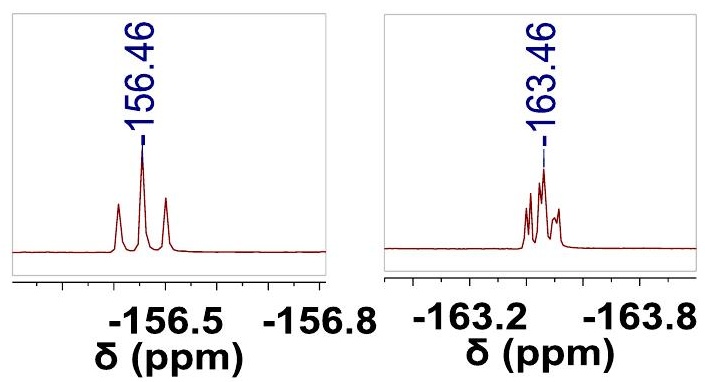

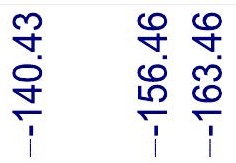
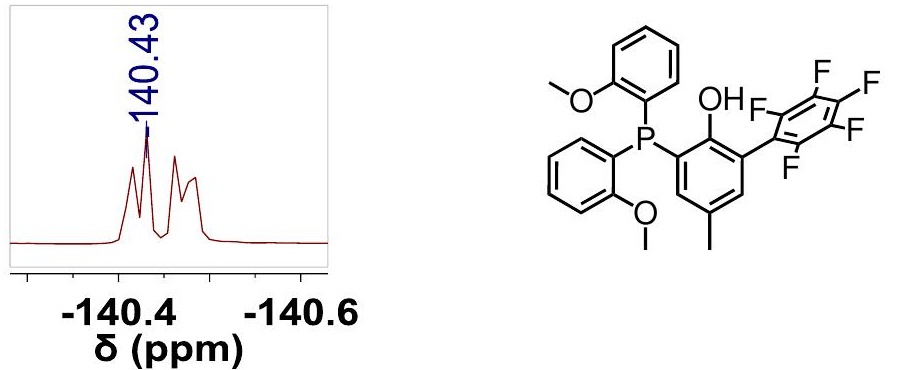

\begin{tabular}{lllllllllll}
\hline & -20 & -40 & -60 & -80 & $\begin{array}{c}-100 \\
\delta(\mathrm{ppm})\end{array}$ & -120 & -140 & -160 & -180 & -21
\end{tabular}

Figure S23. ${ }^{19} \mathrm{~F}$ NMR spectrum $\left(\mathrm{C}_{6} \mathrm{D}_{6}, 470 \mathrm{MHz}\right)$ of compound 3. 


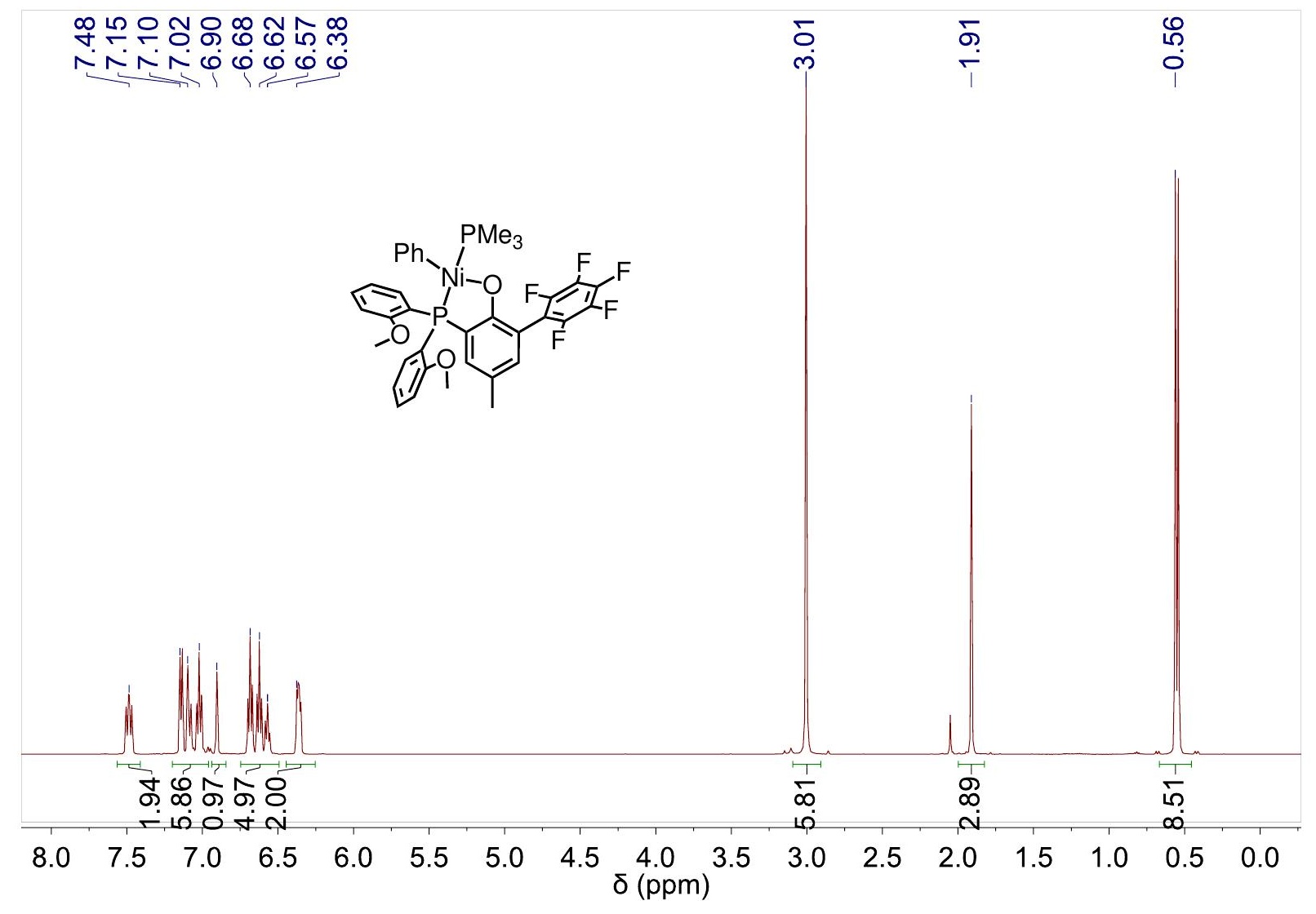

Figure S24. ${ }^{1} \mathrm{H}$ NMR spectrum $\left(\mathrm{C}_{6} \mathrm{D}_{6}, 500 \mathrm{MHz}\right)$ of complex Ni3. 


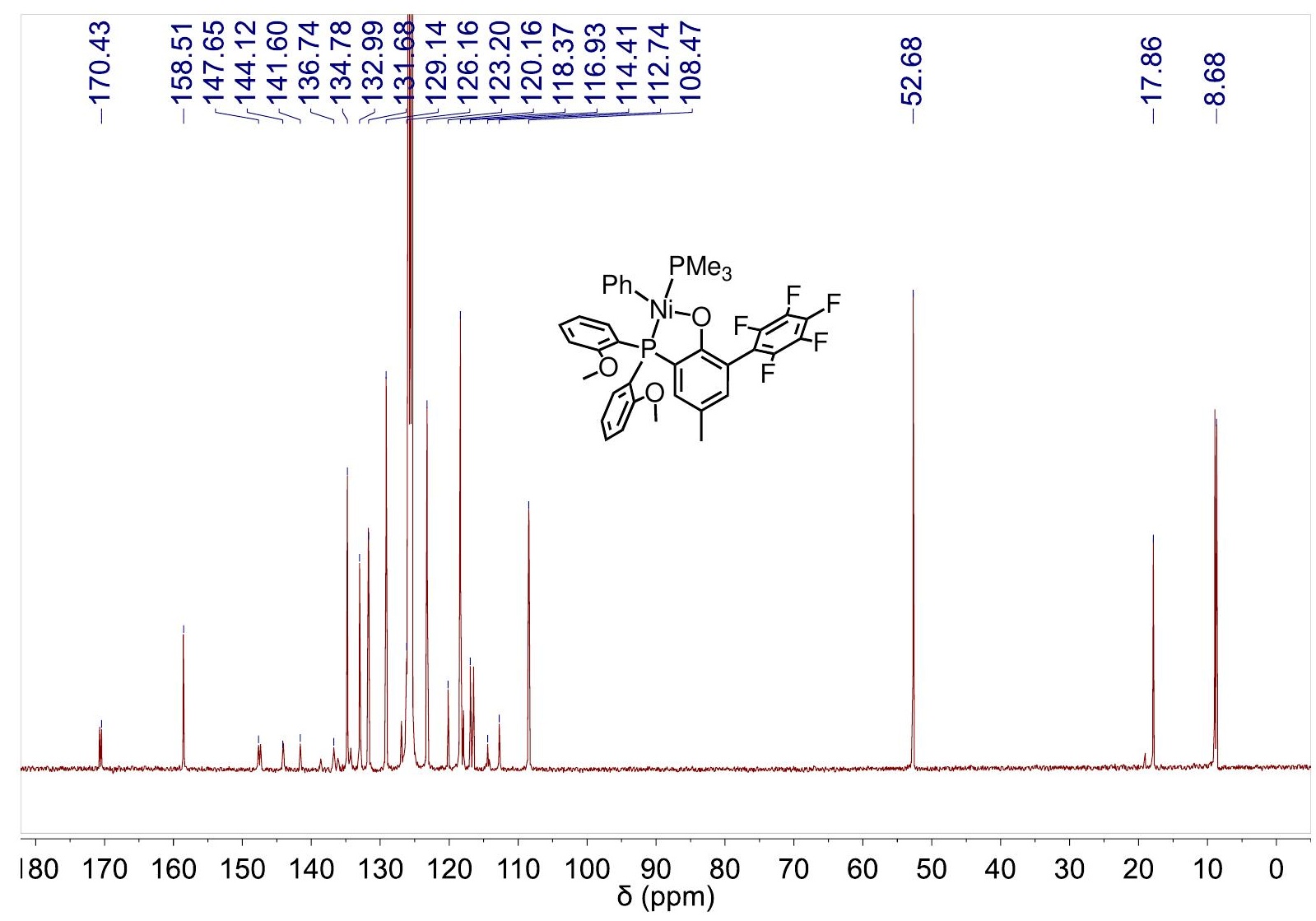

Figure S25. ${ }^{13} \mathrm{C}$ NMR spectrum $\left(\mathrm{C}_{6} \mathrm{D}_{6}, 100 \mathrm{MHz}\right)$ of complex Ni3. 


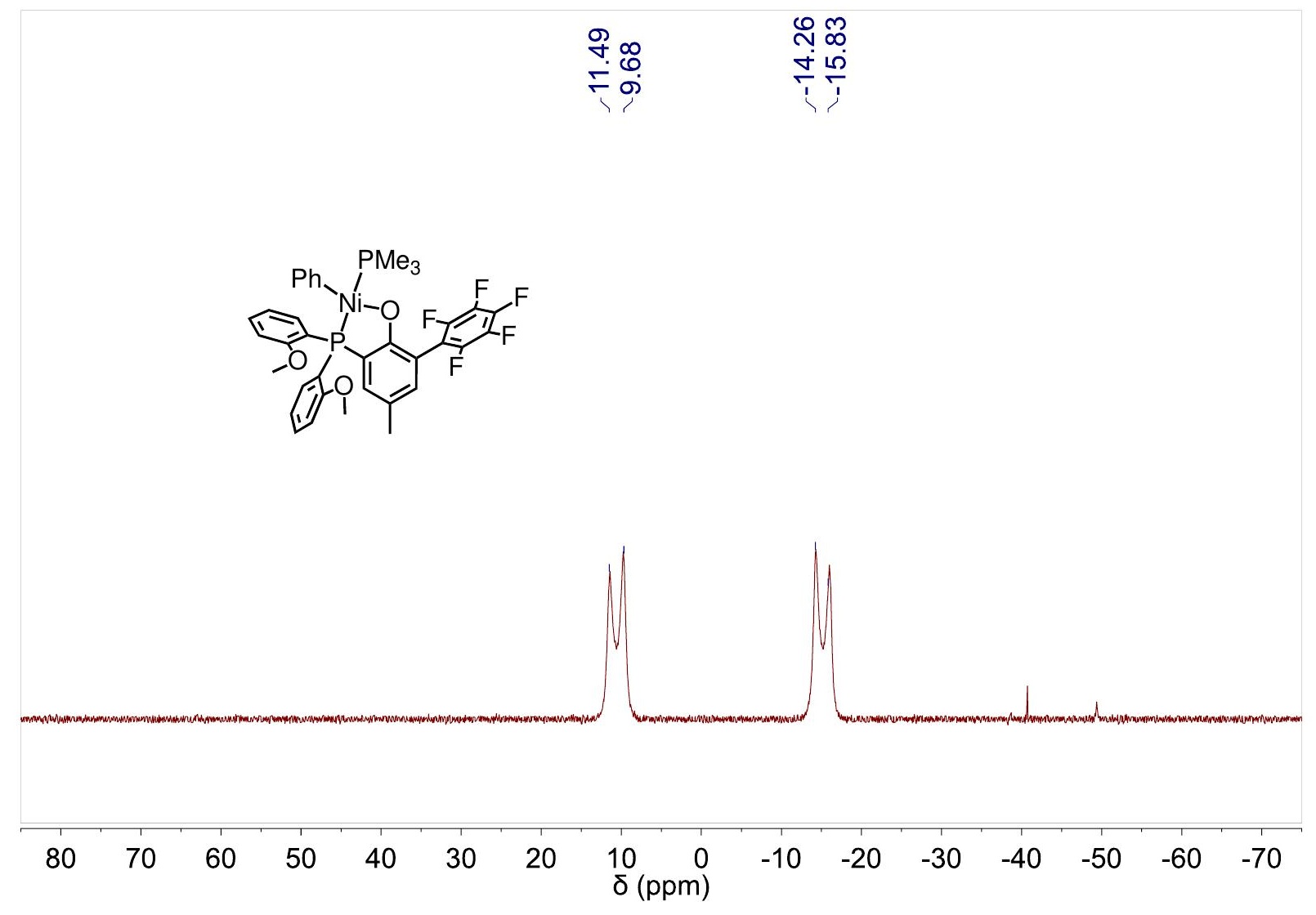

Figure S26. ${ }^{31} \mathrm{P}$ NMR spectrum $\left(\mathrm{C}_{6} \mathrm{D}_{6}, 162 \mathrm{MHz}\right)$ of complex Ni3. 


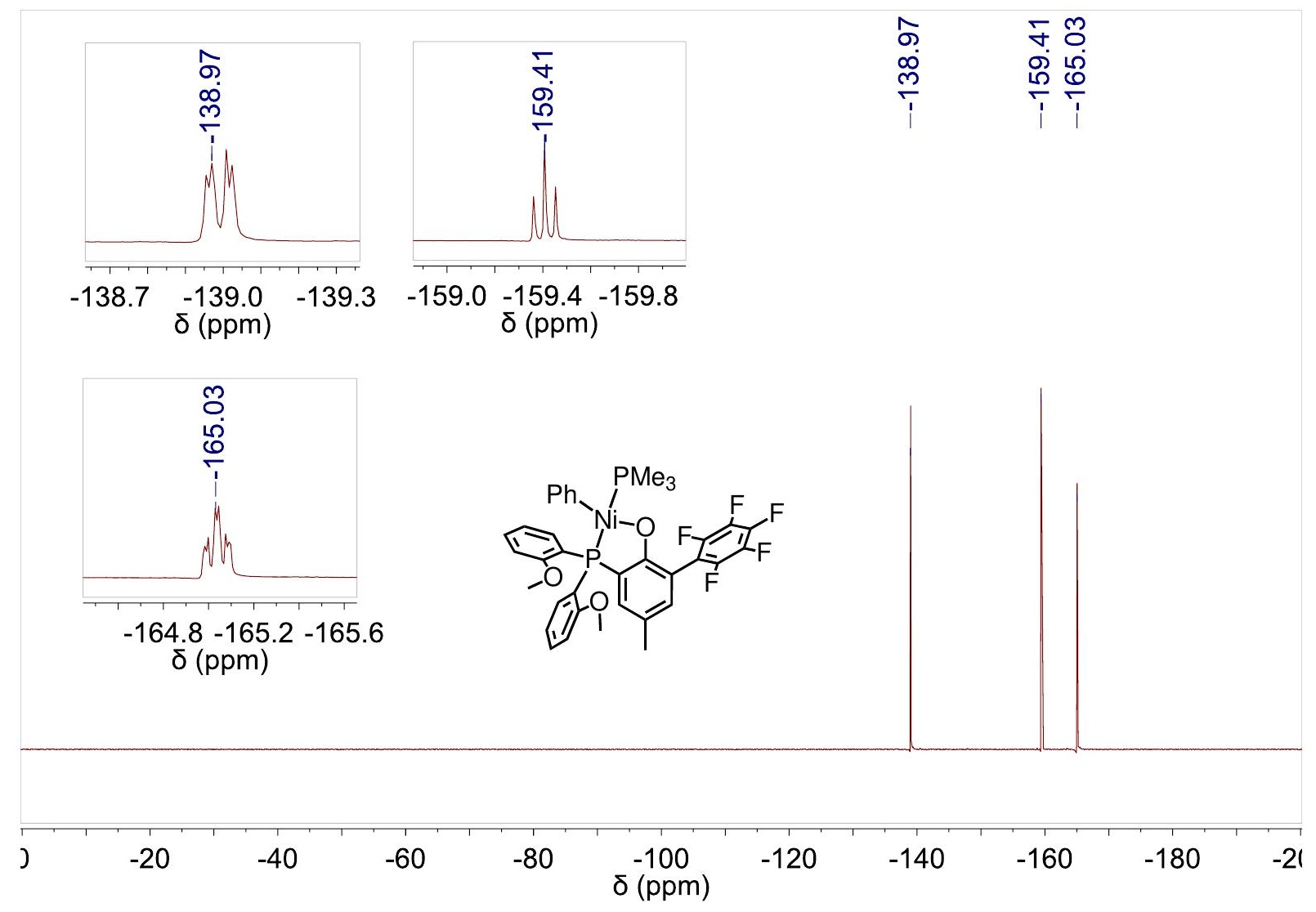

Figure S27. ${ }^{19} \mathrm{~F}$ NMR spectrum $\left(\mathrm{C}_{6} \mathrm{D}_{6}, 470 \mathrm{MHz}\right)$ of complex Ni3. 


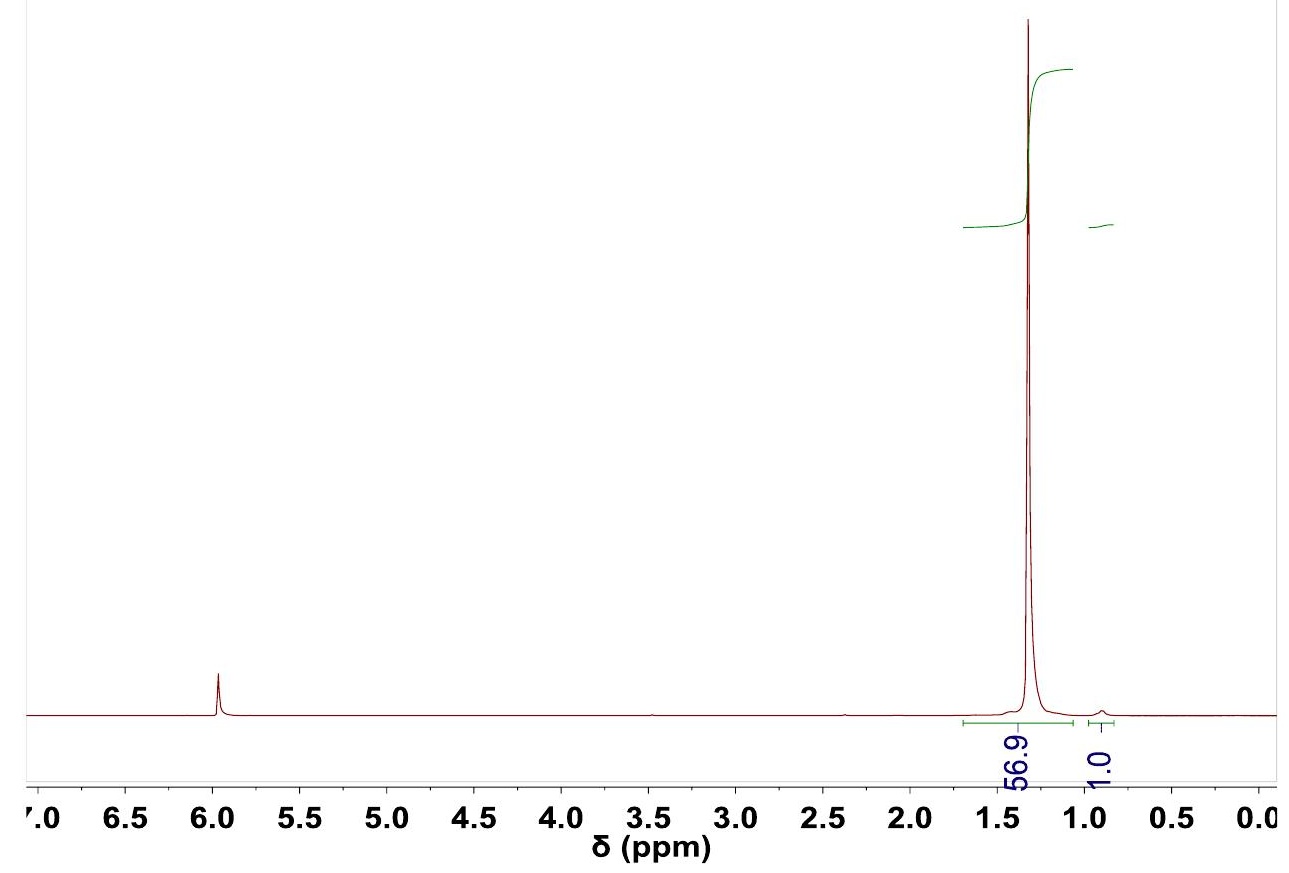

Figure S28. ${ }^{1} \mathrm{H}$ NMR spectrum $\left(\mathrm{C}_{2} \mathrm{D}_{2} \mathrm{Cl}_{4}, 500 \mathrm{MHz}, 120{ }^{\circ} \mathrm{C}\right)$ of polyethylene produced by Ni3$\mathrm{Li}$ at $30^{\circ} \mathrm{C}$ (Table 2 , entry 4$)$.

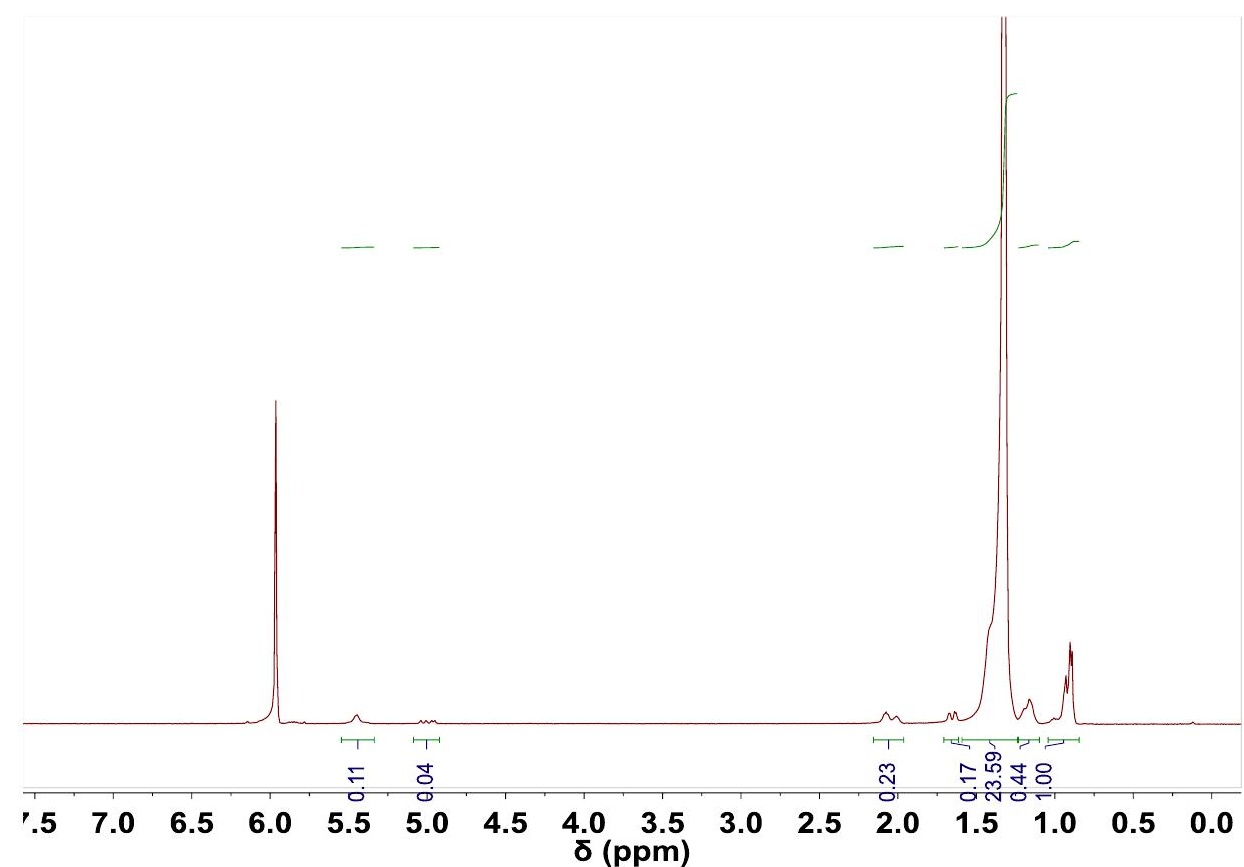

Figure S29. ${ }^{1} \mathrm{H}$ NMR spectrum $\left(\mathrm{C}_{2} \mathrm{D}_{2} \mathrm{Cl}_{4}, 500 \mathrm{MHz}, 120{ }^{\circ} \mathrm{C}\right)$ of polyethylene produced by Ni3$\mathrm{Na}$ at $30^{\circ} \mathrm{C}$ (Table 2, entry 5). 


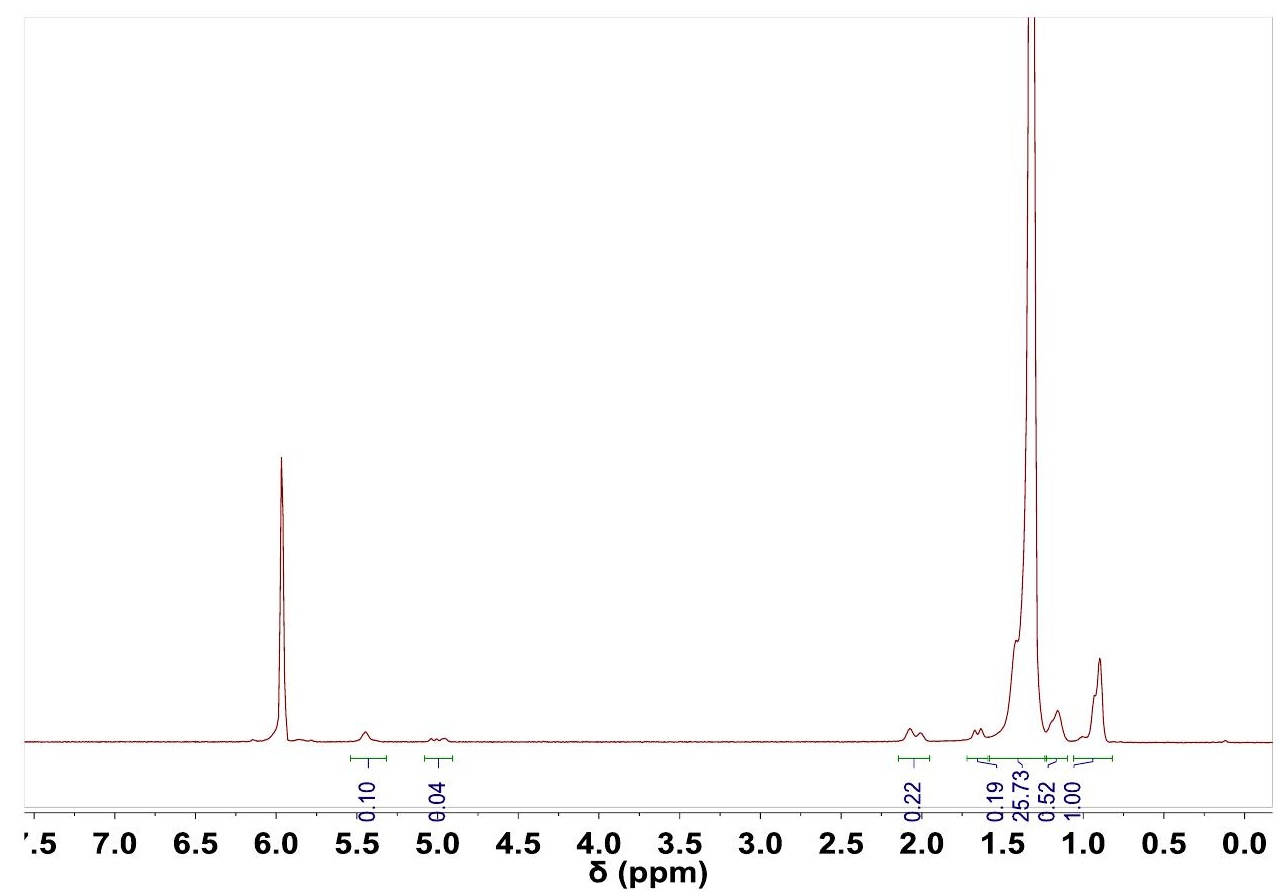

Figure S30. ${ }^{1} \mathrm{H}$ NMR spectrum $\left(\mathrm{C}_{2} \mathrm{D}_{2} \mathrm{Cl}_{4}, 500 \mathrm{MHz}, 120{ }^{\circ} \mathrm{C}\right)$ of polyethylene produced by Ni3$\mathrm{K}$ at $30^{\circ} \mathrm{C}$ (Table 2 , entry 6 ).

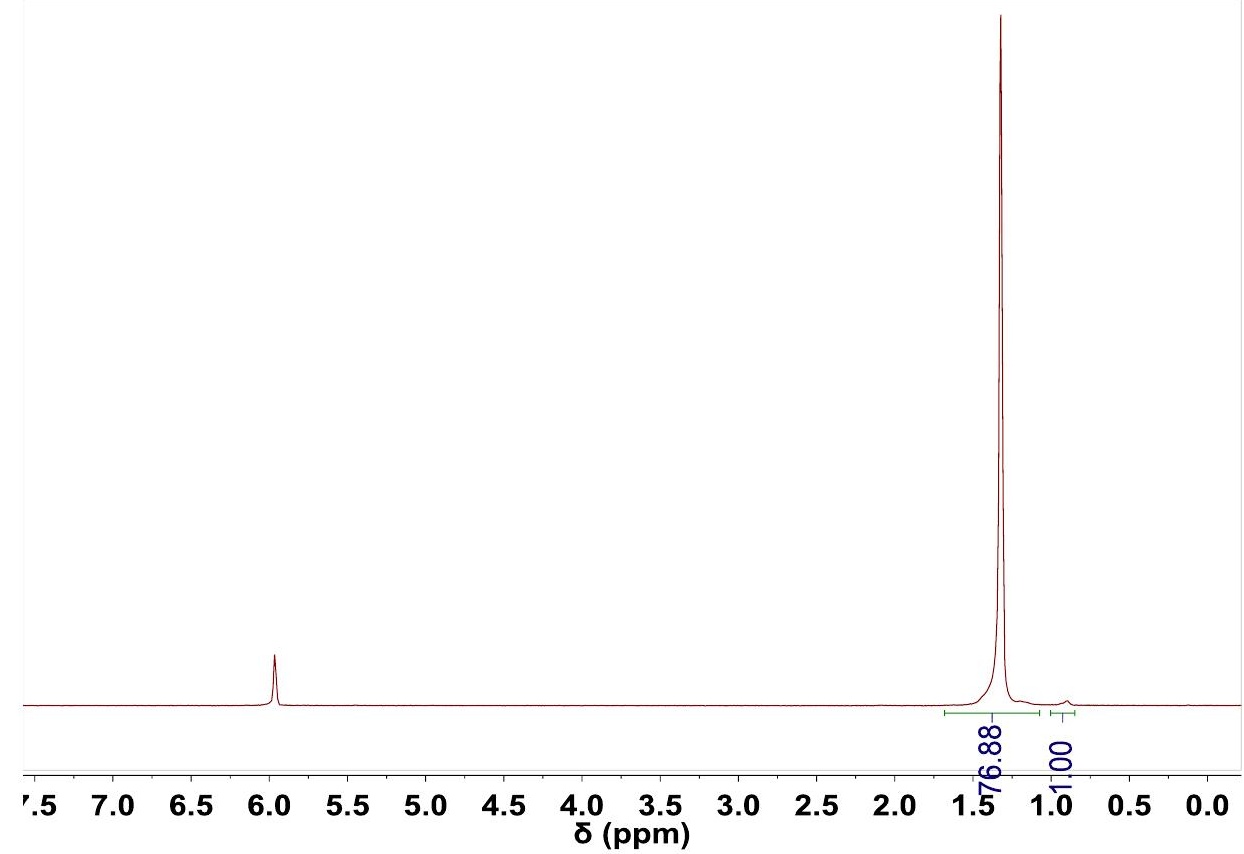

Figure S31. ${ }^{1} \mathrm{H}$ NMR spectrum $\left(\mathrm{C}_{2} \mathrm{D}_{2} \mathrm{Cl}_{4}, 500 \mathrm{MHz}, 120{ }^{\circ} \mathrm{C}\right)$ of polyethylene produced by Ni3Cs at $30{ }^{\circ} \mathrm{C}$ (Table 2, entry 7$)$. 

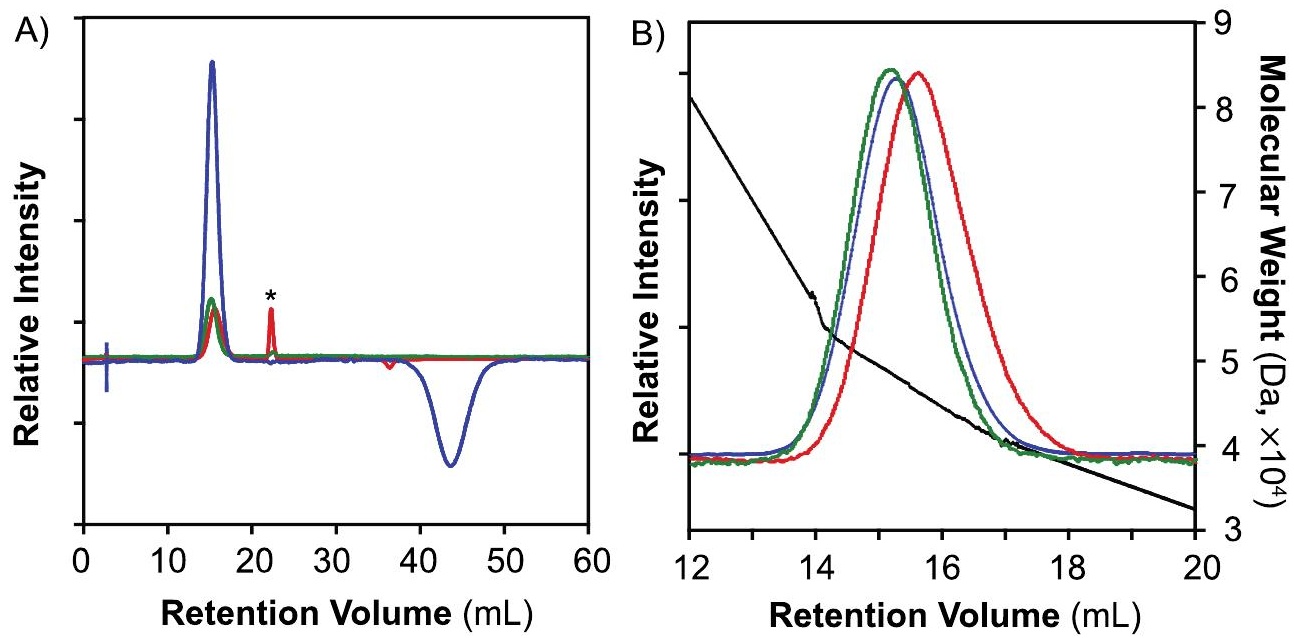

Figure S32. A) GPC of the polyethylene produced by Ni3-Li (Table 2, entry 4) at $30{ }^{\circ} \mathrm{C}$. B) Normalized chromatograms showing the peaks corresponding to the polyethylene product and the molecular weight range (black line). The data were acquired using a triple detector system: red $=$ refractive index, green $=$ right angle light scattering, blue $=$ viscometer. The peak at $\sim 21$ $\mathrm{mL}$ retention volume marked with an asterisk (*) is derived from a contaminant in the GPC column.
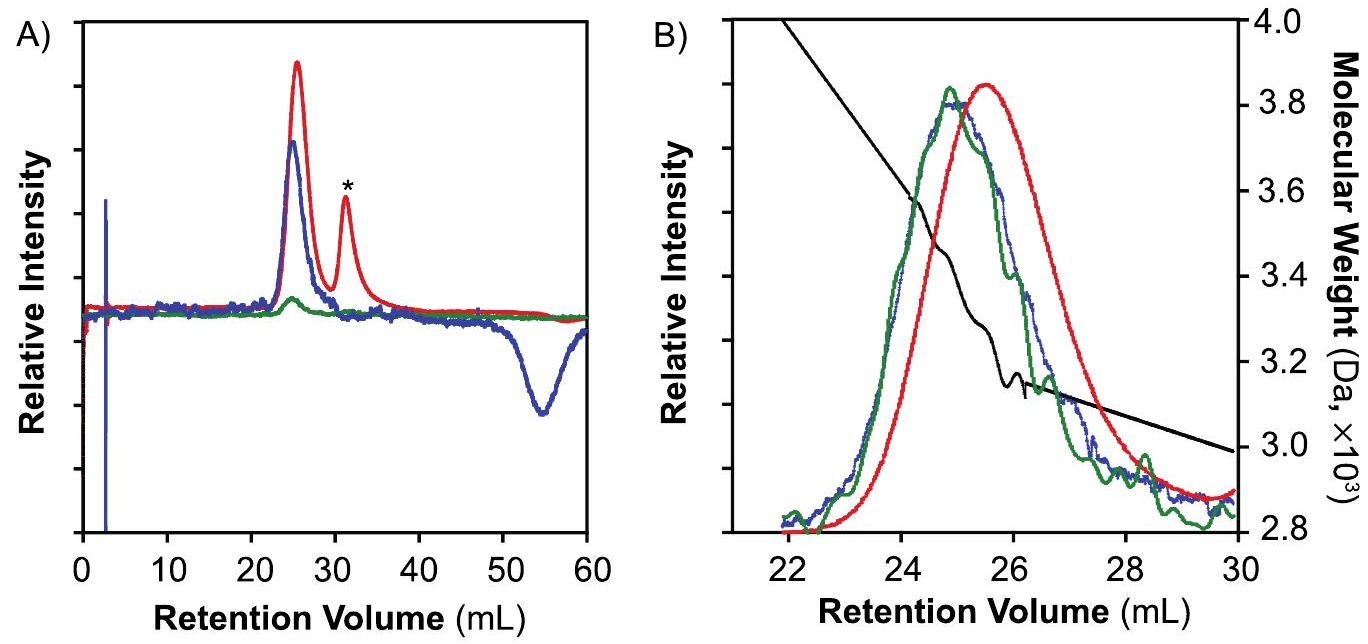

Figure S33. A) GPC of the polyethylene produced by Ni3-Na (Table 2, entry 5) at $30{ }^{\circ} \mathrm{C}$. B) Normalized chromatograms showing the peaks corresponding to the polyethylene product and the molecular weight range (black line). The data were acquired using a triple detector system: red $=$ refractive index, green $=$ right angle light scattering, blue $=$ viscometer. The peak at $\sim 21$ $\mathrm{mL}$ retention volume marked with an asterisk (*) is derived from a contaminant in the GPC column. 

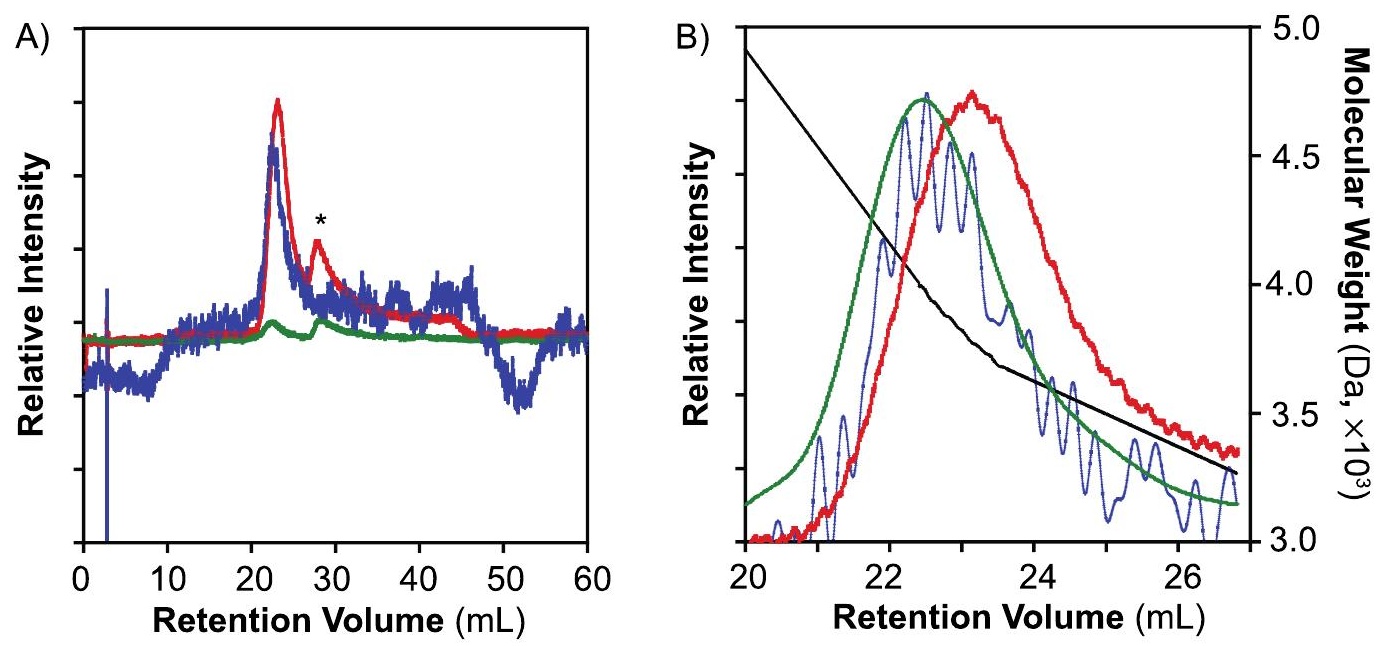

Figure S34. A) GPC of the polyethylene produced by Ni3-K (Table 2, entry 6) at $30{ }^{\circ} \mathrm{C}$. B) Normalized chromatograms showing the peaks corresponding to the polyethylene product and the molecular weight range (black line). The data were acquired using a triple detector system: red $=$ refractive index, green $=$ right angle light scattering, blue $=$ viscometer. The peak at $\sim 21$ $\mathrm{mL}$ retention volume marked with an asterisk (*) is derived from a contaminant in the GPC column.
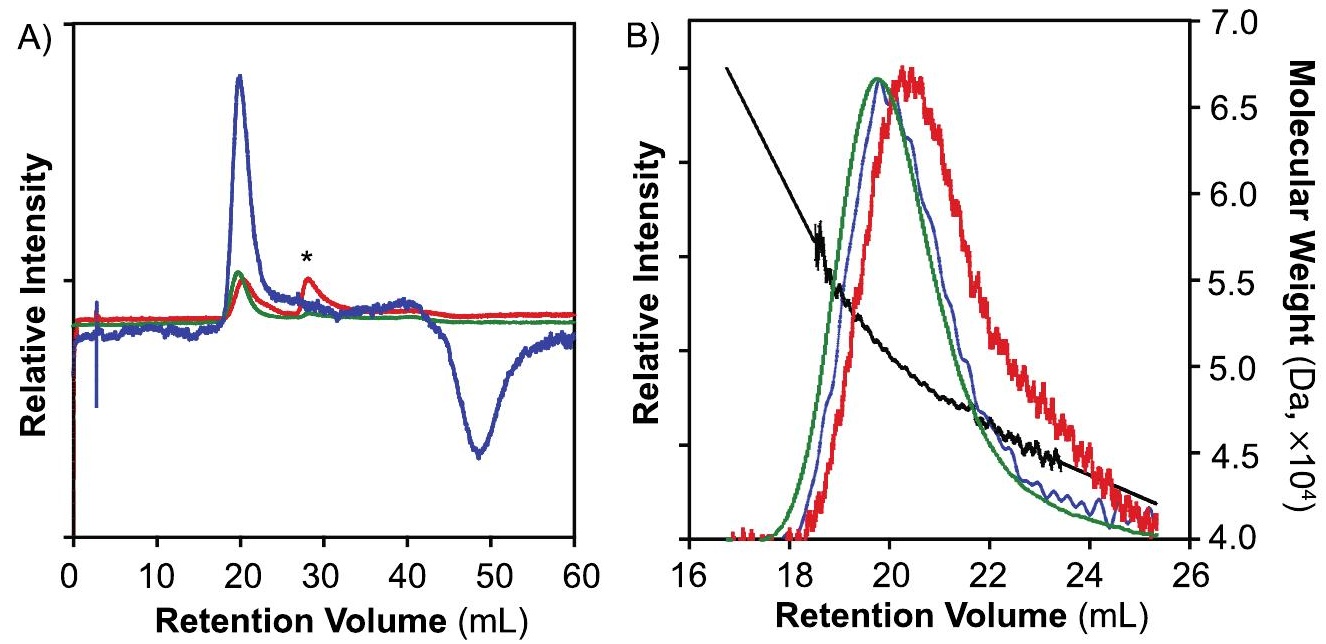

Figure S35. A) GPC of the polyethylene produced by Ni3-Cs (Table 2, entry 7) at $30{ }^{\circ} \mathrm{C}$. B) Normalized chromatograms showing the peaks corresponding to the polyethylene product and the molecular weight range (black line). The data were acquired using a triple detector system: red $=$ refractive index, green $=$ right angle light scattering, blue $=$ viscometer. The peak at $\sim 21$ $\mathrm{mL}$ retention volume marked with an asterisk $(*)$ is derived from a contaminant in the GPC column. 


\section{$\underline{\text { X-ray Data Collection and Refinement }}$}

Single crystals suitable for X-ray diffraction studies were picked out of the crystallization vials and mounted onto Mitogen loops using Paratone oil. The crystals were collected at a $6.0 \mathrm{~cm}$ detector distance at $-150^{\circ} \mathrm{C}$ on a Brucker Apex II diffractometer using Mo $\mathrm{K} \alpha$ radiation $(\lambda=$ $0.71073 \AA$ ). The structures were solved by direct methods using the program SHELXT ${ }^{17}$ and refined by SHELXLE. ${ }^{18-20}$ Hydrogen atoms connected to carbon were placed at idealized positions using standard riding models and refined isotropically. All non-hydrogen atoms were refined anisoptriocally.

Crystals of complex Ni3-Li were grown by layering of pentane into a solution of the nickel complex with $\mathrm{LiBAr}_{4} \mathrm{~F}_{4}$ in a mixture of toluene and $\mathrm{Et}_{2} \mathrm{O}$ at $-30{ }^{\circ} \mathrm{C}$. The asymmetric unit of Ni3-Li contains a toluene (disordered; checkcif " $\mathrm{A}$ " alert regarding small average phenyl $\mathrm{C}-\mathrm{C}$ distance is due to high thermal motion) and pentane molecules that were modeled using the DSR fragment plugin in SHELXLE. Several $\mathrm{CF}_{3}$ groups on the $\mathrm{BAr}_{4}{ }_{4}^{-}$showed rotational disorder and were modeled accordingly.

Crystals of complex Ni3-K were grown by layering of pentane into a solution of the nickel complex with $\mathrm{KBAr}_{4}{ }_{4}$ in a mixture of toluene and $\mathrm{THF}$ at $-30{ }^{\circ} \mathrm{C}$. The asymmetric unit of Ni3-K contains two pentane solvent molecules, one was given full occupancy while the other was given 0.33 occupancy. A disordered THF solvent was coordinated to the potassium center. Several $\mathrm{CF}_{3}$ groups on the $\mathrm{BAr}_{4}{ }_{4}^{-}$showed rotational disorder and were modeled accordingly.

Crystals of complex Ni3-Cs were grown by layering of pentane into a solution of the nickel complex with $\mathrm{CsBAr}_{4}{ }_{4}$ in a mixture of benzene and THF at $-30{ }^{\circ} \mathrm{C}$. A benzene molecule was coordinated to the cesium center. The cesium-PEG group was modeled with positional disorder. Several $\mathrm{CF}_{3}$ groups on the $\mathrm{BAr}^{\mathrm{F}_{4}}{ }^{-}$showed rotational disorder and were modeled accordingly.

The x-ray data for Ni3-Na were obtained from our previous publication. ${ }^{3}$ 
Table S14. Crystal Data and Structure Refinement for Ni3-Li, Ni3-K and Ni3-Cs

\begin{tabular}{|c|c|c|c|}
\hline & $\begin{array}{l}\text { Ni3-Li· } \\
\text { (toluene)(pentane) }\end{array}$ & $\begin{array}{l}\text { Ni3-K(THF) } \\
\text { (pentane })_{x}\end{array}$ & Ni3-Cs(benzene) \\
\hline Empirical Formula & $\begin{array}{l}\mathrm{NiLiC}_{38} \mathrm{H}_{50} \mathrm{O}_{7} \mathrm{P}_{2} \\
\left(\mathrm{BC}_{32} \mathrm{H}_{12} \mathrm{~F}_{24}\right) \\
\left(\mathrm{C}_{7} \mathrm{H}_{8}\right)\left(\mathrm{C}_{5} \mathrm{H}_{12}\right)\end{array}$ & $\begin{array}{l}\mathrm{NiKC}_{38} \mathrm{H}_{50} \mathrm{O}_{7} \mathrm{P}_{2} \\
\left(\mathrm{BC}_{32} \mathrm{H}_{12} \mathrm{~F}_{24}\right)\left(\mathrm{C}_{4} \mathrm{H}_{8} \mathrm{O}\right) \\
\left(\mathrm{C}_{5} \mathrm{H}_{12}\right)_{\mathrm{x}}\end{array}$ & $\begin{array}{l}\mathrm{NiCsC}_{38} \mathrm{H}_{50} \mathrm{O}_{7} \mathrm{P}_{2} \\
\left(\mathrm{BC}_{32} \mathrm{H}_{12} \mathrm{~F}_{24}\right)\left(\mathrm{C}_{6} \mathrm{H}_{6}\right)\end{array}$ \\
\hline Formula Weight & 1773.87 & 1860.00 & 1813.67 \\
\hline Temperature $\left({ }^{\circ} \mathrm{C}\right)$ & -150 & -150 & -150 \\
\hline Wavelength $(\AA)$ & 0.71073 & 0.71073 & 0.71073 \\
\hline Crystal System & Triclinic & Triclinic & Monoclinic \\
\hline Space Group & $\mathrm{P} \overline{1}$ & $\mathrm{P} \overline{1}$ & $\mathrm{P} 2(1) / \mathrm{n}$ \\
\hline $\begin{array}{l}\text { Unit Cell Dimensions } \\
\quad a(\AA) \\
b(\AA) \\
c(\AA) \\
\alpha\left(\left(^{\circ}\right)\right. \\
\beta\left({ }^{\circ}\right) \\
\gamma\left(\left(^{\circ}\right)\right.\end{array}$ & $\begin{array}{l}14.2489(12) \\
14.2542(12) \\
20.5358(18) \\
84.3560(10) \\
86.9020(10) \\
86.2420(10)\end{array}$ & $\begin{array}{l}13.151(3) \\
13.356(3) \\
24.585(6) \\
86.239(2) \\
81.170(3) \\
87.895(3)\end{array}$ & $\begin{array}{l}17.3656(15) \\
23.606(2) \\
20.3261(18) \\
90 \\
103.6820(10) \\
90\end{array}$ \\
\hline Volume $\left(\AA^{3}\right)$ & 4137.1(6) & $4256.1(17)$ & $8095.9(12)$ \\
\hline Z, Calculated Density $\left(\mathrm{Mg} / \mathrm{m}^{3}\right)$ & $2,1.424$ & $2,1.394$ & $4,1.488$ \\
\hline Absorption Coefficient $\left(\mathrm{mm}^{-1}\right)$ & 0.38 & 0.418 & 0.828 \\
\hline $\mathbf{F}(\mathbf{0 0 0})$ & 1820 & 1836 & 3656 \\
\hline Theta Range for Data Collection $\left(^{\circ}\right)$ & 1.671 to 24.713 & 0.840 to 25.350 & 1.344 to 24.711 \\
\hline Limiting Indices & $\begin{array}{l}-15 \leq \mathrm{h} \leq 16 \\
-15 \leq \mathrm{k} \leq 16 \\
-14 \leq 1 \leq 24\end{array}$ & $\begin{array}{l}-15 \leq \mathrm{h} \leq 15 \\
-16 \leq \mathrm{k} \leq 16 \\
-29 \leq 1 \leq 29\end{array}$ & $\begin{array}{l}-20 \leq \mathrm{h} \leq 20 \\
-24 \leq \mathrm{k} \leq 27 \\
-19 \leq 1 \leq 23\end{array}$ \\
\hline Reflections Collected/ Unique & $\begin{array}{l}20662 / 13855 \\
{[R(\text { int })=0.0177]}\end{array}$ & $\begin{array}{l}50354 / 15533 \\
{[R(\text { int })=0.0429]}\end{array}$ & $\begin{array}{l}40225 / 13791 \\
{[R(\text { int })=0.0259]}\end{array}$ \\
\hline Data/ Restraints/ Parameters & 13855 / $203 / 1005$ & $15533 / 213 / 998$ & 13791 / 2257 / 962 \\
\hline Goodness of Fit on $F^{2}$ & 1.030 & 1.038 & 1.035 \\
\hline $\begin{array}{l}\text { Final R Indices } \\
{[\mathrm{I}>2 \sigma(\mathrm{I})]}\end{array}$ & $\begin{array}{l}\mathrm{R}_{1}=0.0706 \\
\mathrm{wR}_{2}=0.1979\end{array}$ & $\begin{array}{l}\mathrm{R}_{1}=0.0985 \\
\mathrm{wR}_{2}=0.2889\end{array}$ & $\begin{array}{l}\mathrm{R}_{1}=0.1011 \\
\mathrm{wR}_{2}=0.2712\end{array}$ \\
\hline R Indices (All Data)* & $\begin{array}{l}\mathrm{R}_{1}=0.0809 \\
\mathrm{wR}_{2}=0.2086\end{array}$ & $\begin{array}{l}\mathrm{R}_{1}=0.1301 \\
\mathrm{wR}_{2}=0.3224\end{array}$ & $\begin{array}{l}\mathrm{R}_{1}=0.1203 \\
\mathrm{wR}_{2}=0.2907\end{array}$ \\
\hline Largest Diff. Peak and Hole $\left(\mathrm{e} \AA^{-3}\right)$ & 1.483 and -0.975 & 2.294 and -2.017 & 2.761 and -1.275 \\
\hline
\end{tabular}




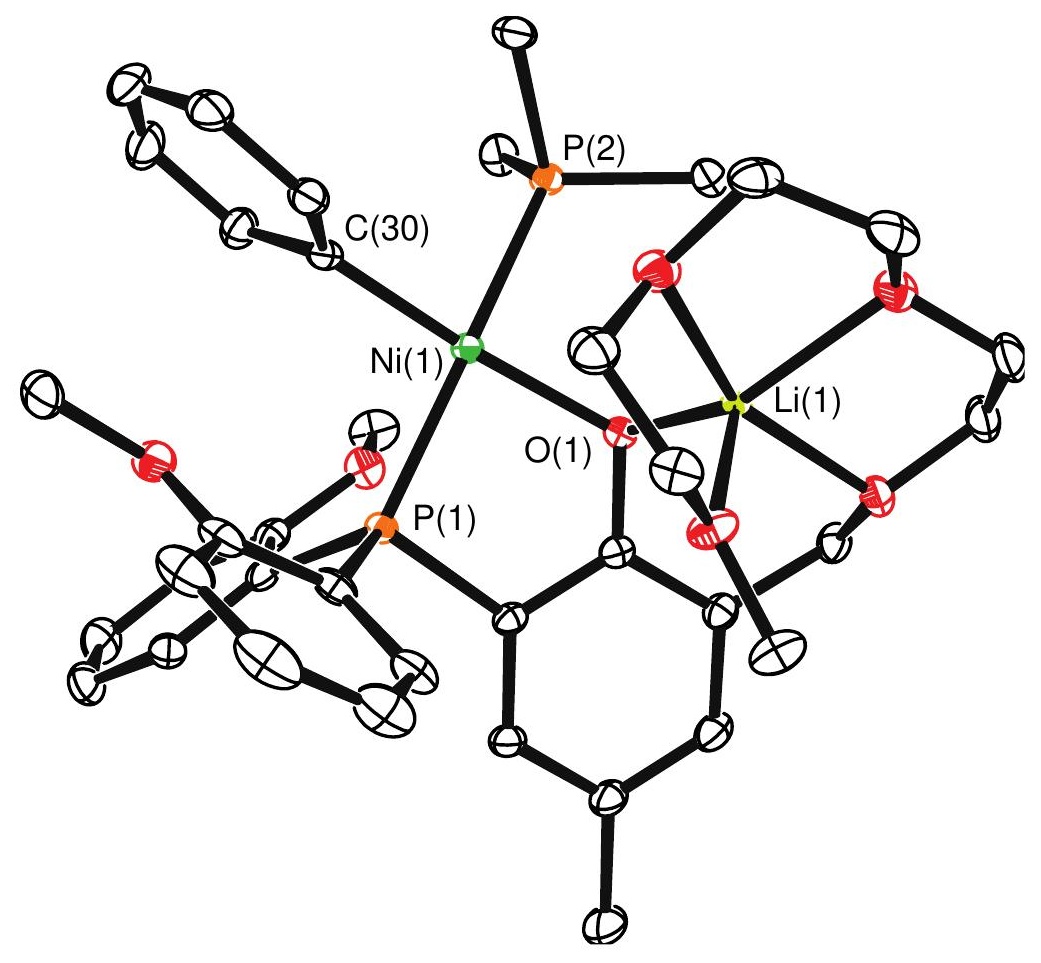

Figure S36. Crystallographic asymmetric unit showing complex Ni3-Li (ORTEP view, displacement ellipsoids drawn at 50\% probability level). Hydrogen atoms and pentane solvent have been omitted for clarity. Atom colors: green $=$ nickel, orange $=$ phosphorus, yellow $=$ lithium, red $=$ oxygen, black $=$ carbon . 


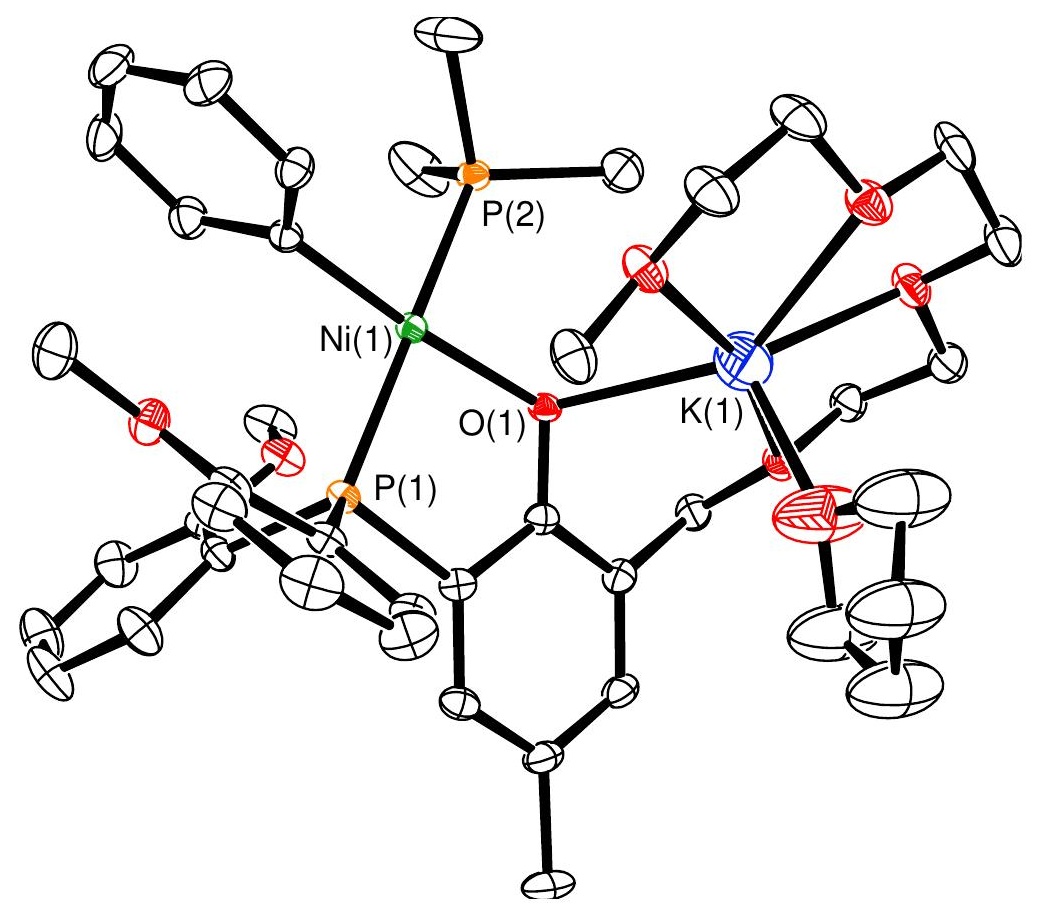

Figure S37. Crystallographic asymmetric unit showing complex Ni3-K (ORTEP view, displacement ellipsoids drawn at 50\% probability level). Hydrogen atoms and pentane solvent have been omitted for clarity. Atom colors: green $=$ nickel, orange $=$ phosphorus, blue $=$ potassium, red $=$ oxygen, black $=$ carbon. 


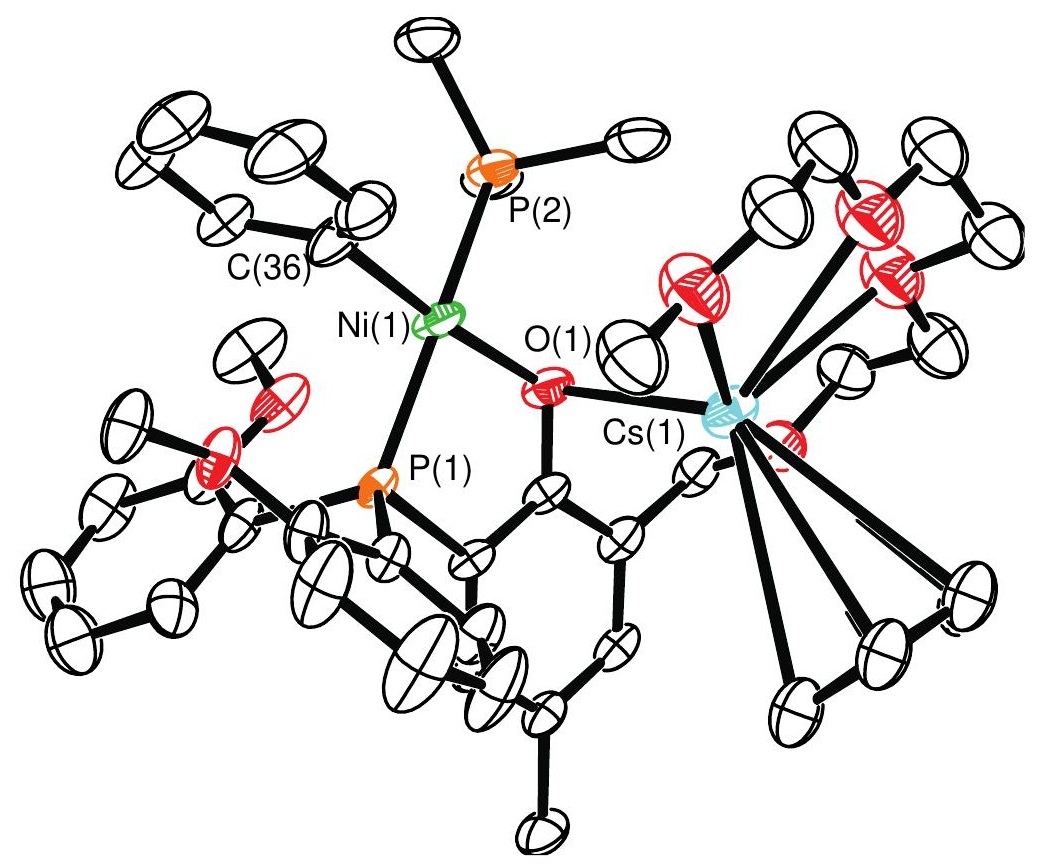

Figure S38. Crystallographic asymmetric unit showing complex Ni3-Cs (ORTEP view, displacement ellipsoids drawn at 50\% probability level). Hydrogen atoms and pentane solvent have been omitted for clarity. Atom colors: green $=$ nickel, orange $=$ phosphorus, cyan $=$ cesium, red $=$ oxygen, black $=$ carbon. 


\section{Additional Notes on Polymerization Studies}

To obtain consistent polymer yields from run to run, the amount of catalyst used in each run must be kept as consistent as possible. Since $0.5 \mu \mathrm{mol}$ of the Ni3 catalyst weighs only $0.37 \mathrm{mg}$, it is extremely difficult to weigh out exactly this amount using a standard analytical balance. To minimize errors due to weighing inconsistencies, we used a batch catalyst preparation method. First, we weighed out $37 \mathrm{mg}(50 \mu \mathrm{mol})$ of the catalyst and then dissolved it into $50 \mathrm{~mL}$ of toluene. This solution was divided equally into 10 vials so that each vial contained $5 \mu \mathrm{mol}$ of catalyst. Next, we combined each $5 \mu \mathrm{mol}$ of catalyst with $20 \mathrm{~mL}$ of toluene and partitioned this $25 \mathrm{~mL}$ mixture into 10 vials so that each vial contained $0.5 \mu \mathrm{mol}$ of catalyst. Finally, each vial was dried completely under vacuum and stored in a refrigerator inside the drybox until ready for use.

For reactions using $0.1 \mu \mathrm{mol}$ of the $\mathbf{N i 3}$ catalyst, the catalyst preparation was similar to the procedure mentioned above.

For all polymerization reactions, except ones that were performed to determine the temperature profiles, the reaction temperature was controlled by manual cooling of the reactor with an air stream when the reactor increases more than $5^{\circ} \mathrm{C}$ above the starting temperature.

To clean the Parr reactor, the vessel was washed with hot toluene $\left(80^{\circ} \mathrm{C}\right)$ to remove the polymer sample from the previous run and rinsed with acetone before drying under vacuum for at least 1 $\mathrm{h}$ to remove trace amounts of water. 
Table S15. Cis and Trans Isomer Distribution ${ }^{a}$

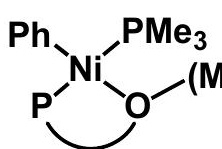

Isomer A

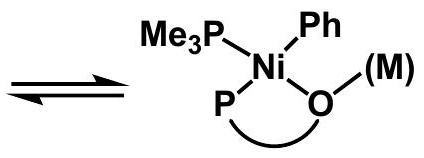

Isomer B

\begin{tabular}{ccccc}
\hline Complex & $\begin{array}{c}\text { Ratio } \\
(\mathrm{A}: \mathrm{B})\end{array}$ & $\begin{array}{c}\boldsymbol{K} \text { eq } \\
([\mathrm{B}] /[\mathrm{A}])\end{array}$ & $\begin{array}{c}\Delta \mathbf{G}_{\text {obs }}{ }^{b} \\
(\mathrm{kcal} / \mathrm{mol})\end{array}$ & $\begin{array}{c}\Delta \mathbf{G}_{\mathbf{c a l c}}{ }^{c} \\
(\mathrm{kcal} / \mathrm{mol})\end{array}$ \\
\hline Ni1 & $100: 0$ & 0 & - & 9.3 \\
Ni2 & $100: 0$ & 0 & - & 6.6 \\
Ni3 & $100: 0$ & 0 & - & 6.2 \\
Ni3-Li & $92: 8$ & 0.1 & 1.4 & 2.0 \\
Ni3-Na & $10: 90$ & 9.0 & -1.3 & -2.8 \\
Ni3-K & $12: 88$ & 7.3 & -1.2 & -1.1 \\
Ni3-Cs & $65: 35$ & 0.5 & 0.4 & 3.3
\end{tabular}

${ }^{a}$ The relative amounts of isomers A and B were determined from the ${ }^{31} \mathrm{P}$ NMR spectra of the nickel complexes in a mixture of toluene- $d_{8} /$ diethyl ether (4:1) (see Figure 6). ${ }^{b}$ The free energy change associated with isomerization of A to $\mathrm{B}$ was determined based on the experimentally observed equilibrium constant $K$ eq using the equation: $\Delta \mathrm{G}=-\mathrm{RT}$ $\ln K_{\text {eq }}$, where $\mathrm{T}$ was $298 \mathrm{~K}^{c}$ The calculations were performed using B3LYP with the 6-31G(d) basis set for C, H, O, $\mathrm{P}, \mathrm{Li}, \mathrm{Na}$, and $\mathrm{K}$ and the Lanl2dz basis set for $\mathrm{Ni}$ and $\mathrm{Cs}$ in the gas phase. 

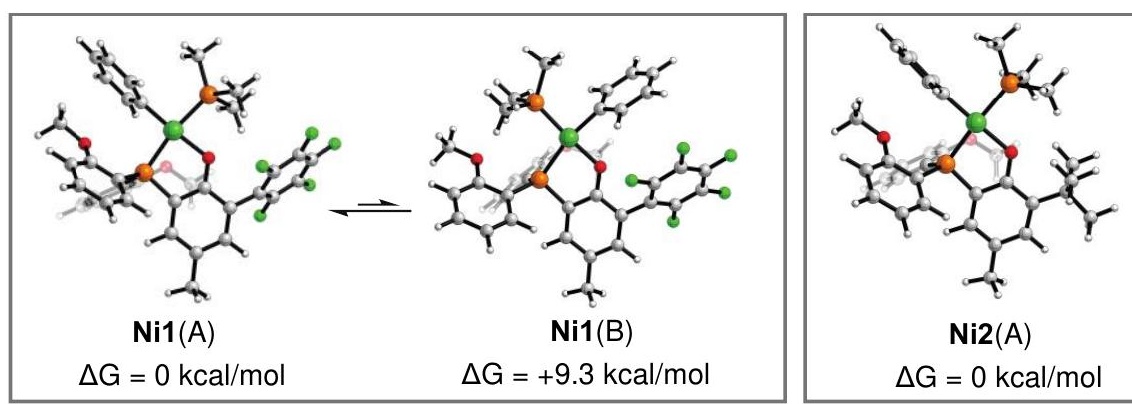

$\mathrm{Ni3}(\mathrm{A})$

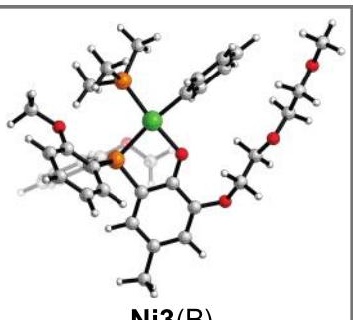

$\mathrm{Ni3}(\mathrm{B})$

$\Delta \mathrm{G}=+6.2 \mathrm{kcal} / \mathrm{mol}$

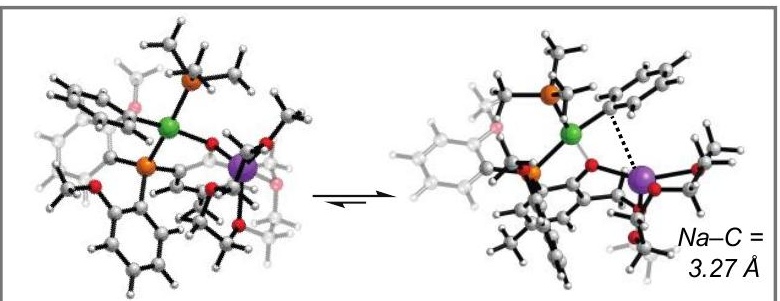

$\mathrm{Ni3}-\mathrm{Na}(\mathrm{A})$

$\Delta \mathrm{G}=+2.8 \mathrm{kcal} / \mathrm{mol}$

$\mathrm{Ni3}-\mathrm{Na}(\mathrm{B})$

$\Delta \mathrm{G}=0 \mathrm{kcal} / \mathrm{mol}$

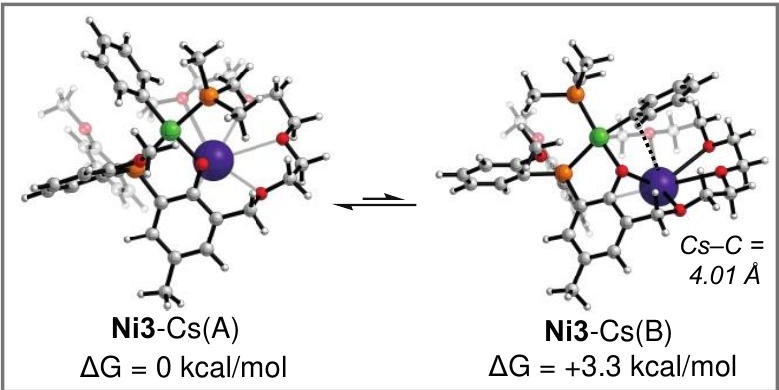

$\mathrm{Ni2}(\mathrm{A})$
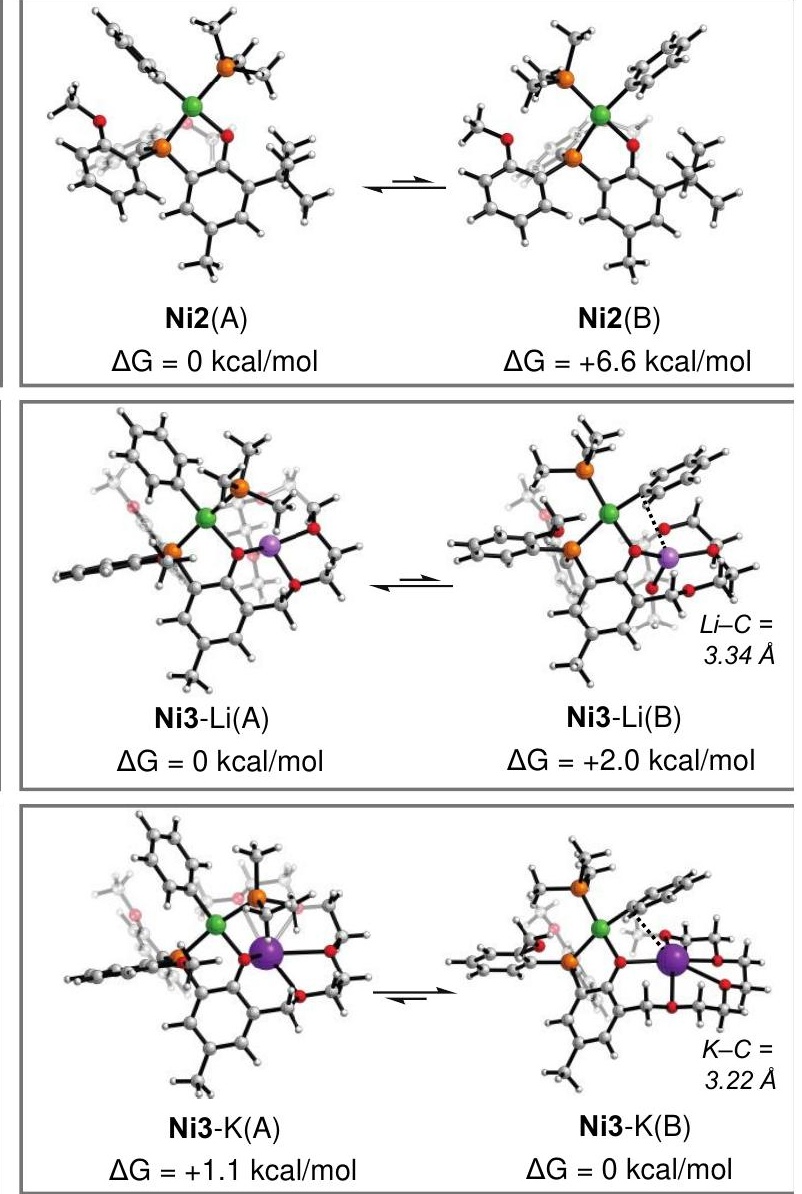

Ni2(B)

\section{$\Delta \mathrm{G}=0 \mathrm{kcal} / \mathrm{mol}$}

Figure S39. DFT optimized structures of the nickel complexes. All calculations were performed using B3LYP with the 6-31G(d) basis set for C, H, O, P, Li, Na, and K and Lanl2dz for Ni and Cs in the gas phase. 

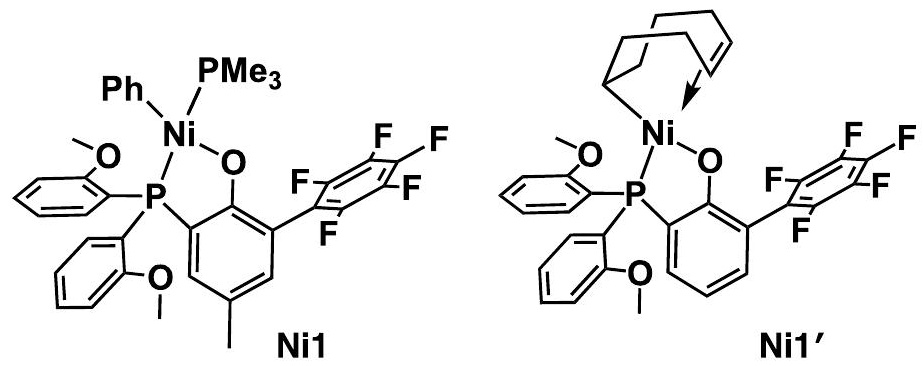

Chart S1. Chemical structures of complexes Ni1 (left) and Ni1' (right). The crystallographic data for Ni1'21 was reported previously and used for structural comparisons in this work. 


\section{$\underline{\text { References }}$}

(1) Shimizu, F.; Xin, S.; Tanna, A.; S., G.; Matsubara, K. U.S. Patent 8618319 B2. 2013.

(2) Hirose, K. A Practical Guide for the Determination of Binding Constants. J. Incl. Phenom. Macrocycl. Chem., 2001, 39 (3), 193-209.

(3) Tran, T. V.; Nguyen, Y. H.; Do, L. H. Development of Highly Productive Nickel-Sodium Phenoxyphosphine Ethylene Polymerization Catalysts and their Reaction Temperature Profiles. Polym. Chem., 2019, 10 (27), 3718-3721.

(4) Gates, D. P.; Svejda, S. A.; Oñate, E.; Killian, C. M.; Johnson, L. K.; White, P. S.; Brookhart, M. Synthesis of Branched Polyethylene Using ( $\alpha$-Diimine)nickel(II) Catalysts: Influence of Temperature, Ethylene Pressure, and Ligand Structure on Polymer Properties.

Macromolecules, 2000, 33 (7), 2320-2334.

(5) Rhinehart, J. L.; Brown, L. A.; Long, B. K. A Robust Ni(II) $\alpha$-Diimine Catalyst for High Temperature Ethylene Polymerization. J. Am. Chem. Soc., 2013, 135 (44), 16316-16319.

(6) Chen, M.; Chen, C. A Versatile Ligand Platform for Palladium- and Nickel-Catalyzed Ethylene Copolymerization with Polar Monomers. Angew. Chem., Int. Ed., 2018, 57 (12), 3094-3098.

(7) Zhou, X.; Bontemps, S.; Jordan, R. F. Base-Free Phosphine-Sulfonate Nickel Benzyl Complexes. Organometallics, 2008, 27 (19), 4821-4824.

(8) Delferro, M.; McInnis, J. P.; Marks, T. J. Ethylene Polymerization Characteristics of an Electron-Deficient Nickel(II) Phenoxyiminato Catalyst Modulated by Non-Innocent Intramolecular Hydrogen Bonding. Organometallics, 2010, 29 (21), 5040-5049.

(9) Kenyon, P.; Mecking, S. Pentafluorosulfanyl Substituents in Polymerization Catalysis. J. Am. Chem. Soc., 2017, 139 (39), 13786-13790.

(10) Zhang, Y.; Mu, H.; Pan, L.; Wang, X.; Li, Y. Robust Bulky [P,O] Neutral Nickel Catalysts for Copolymerization of Ethylene with Polar Vinyl Monomers. ACS Catal., 2018, 8 59635976.

(11) Tao, W.-j.; Nakano, R.; Ito, S.; Nozaki, K. Copolymerization of Ethylene and Polar Monomers by Using Ni/IzQO Catalysts. Angew. Chem., Int. Ed., 2016, 55 (8), 2835-2839.

(12) Kocen, A. L.; Brookhart, M.; Daugulis, O. A Highly Active Ni(II)-Triadamantylphosphine Catalyst for Ultrahigh-Molecular-Weight Polyethylene Synthesis. Nature Commun., 2019, $10(1), 438$.

(13) Camacho, D. H.; Salo, E. V.; Ziller, J. W.; Guan, Z. Cyclophane-Based Highly Active LateTransition-Metal Catalysts for Ethylene Polymerization. Angew. Chem., Int. Ed., 2004, 43 (14), 1821-1825.

(14) Liu, F.-S.; Hu, H.-B.; Xu, Y.; Guo, L.-H.; Zai, S.-B.; Song, K.-M.; Gao, H.-Y.; Zhang, L.; Zhu, F.-M.; Wu, Q. Thermostable $\alpha$-Diimine Nickel(II) Catalyst for Ethylene Polymerization: Effects of the Substituted Backbone Structure on Catalytic Properties and Branching Structure of Polyethylene. Macromolecules, 2009, 42 (20), 7789-7796.

(15) Chen, M.; Chen, C. Rational Design of High-Performance Phosphine Sulfonate Nickel Catalysts for Ethylene Polymerization and Copolymerization with Polar Monomers. ACS Catal., 2017, 7 (2), 1308-1312.

(16) Fu, X.; Zhang, L.; Tanaka, R.; Shiono, T.; Cai, Z. Highly Robust Nickel Catalysts Containing Anilinonaphthoquinone Ligand for Copolymerization of Ethylene and Polar Monomers. Macromolecules, 2017, 50 (23), 9216-9221. 
(17) Chen, Z.; Zhao, X.; Gong, X.; Xu, D.; Ma, Y. Macrocyclic Trinuclear Nickel Phenoxyimine Catalysts for High-Temperature Polymerization of Ethylene and Isospecific Polymerization of Propylene. Macromolecules, 2017, 50 (17), 6561-6568.

(18) Poater, A.; Cosenza, B.; Correa, A.; Giudice, S.; Ragone, F.; Scarano, V.; Cavallo, L. SambVca: A Web Application for the Calculation of the Buried Volume of $N$-Heterocyclic Carbene Ligands. Eur. J. Inorg. Chem., 2009, 2009 (13), 1759-1766.

(19) Poater, A.; Ragone, F.; Giudice, S.; Costabile, C.; Dorta, R.; Nolan, S. P.; Cavallo, L. Thermodynamics of $N$-Heterocyclic Carbene Dimerization: The Balance of Sterics and Electronics. Organometallics, 2008, 27 (12), 2679-2681.

(20) Poater, A.; Ragone, F.; Mariz, R.; Dorta, R.; Cavallo, L. Comparing the Enantioselective Power of Steric and Electrostatic Effects in Transition-Metal-Catalyzed Asymmetric Synthesis. Chem. Eur. J., 2010, 16 (48), 14348-14353.

(21) Xin, B. S.; Sato, N.; Tanna, A.; Oishi, Y.; Konishi, Y.; Shimizu, F. Nickel Catalyzed Copolymerization of Ethylene and Alkyl Acrylates. J. Am. Chem. Soc., 2017, 139 (10), 3611-3614. 